\title{
Singular Limit Solutions for a 4-dimensional Semilinear Elliptic System of Liouville Type
}

\begin{abstract}
Sami Baraket*, Imen Bazarbacha, Rima Chetouane and Abdellatif Messaoudi
Abstract. We consider the existence of singular limit solutions for a nonlinear elliptic system of Liouville type with Navier boundary conditions. We use the nonlinear domain decomposition method and a Pohozaev type identity.
\end{abstract}

\section{Introduction and statement of the results}

Let $\Omega \subset \mathbb{R}^{4}$ be a regular bounded open domain in $\mathbb{R}^{4}$. We consider the following elliptic system

$$
\begin{cases}\Delta^{2} u_{1}=\rho^{4} e^{\gamma u_{1}+(1-\gamma) u_{2}} & \text { in } \Omega, \\ \Delta^{2} u_{2}=\rho^{4} e^{\xi u_{2}+(1-\xi) u_{1}} & \text { in } \Omega, \\ u_{i}=\Delta u_{i}=0, i=1,2 & \text { on } \partial \Omega\end{cases}
$$

here $\gamma, \xi$ and $\rho$ are constants. We assume that $\gamma, \xi \in(0,1)$ such that $\gamma+\xi>1$. So in all the following, we have

$$
\frac{1-\xi}{\gamma}, \frac{1-\gamma}{\xi} \in(0,1)
$$

We are interested in the study of the existence of solutions with singular limits as the parameter $\rho$ tends to 0 .

The system (1.1) is a natural generalization of the equation

$$
\Delta^{2} u=6 e^{4 u} \quad \text { in } \mathbb{R}^{4}
$$

Equation (1.2) is invariant under translation, rotation, dilatation in the Euclidean space and the Kelvin transforms. In [15], Lin proved the following important classification result of finite-mass solutions of equation 1.2 .

Received April 2, 2019; Accepted December 3, 2019.

Communicated by Jann-Long Chern.

2010 Mathematics Subject Classification. 35J60, 53C21, 58J05.

Key words and phrases. Liouville type system, singular limit solution, nonlinear domain decomposition method, Green's function, Pohozaev identity.

*Corresponding author. 
Theorem 1.1. [15] Let $u$ be a solution of 1.2 , satisfying the finite-mass condition

$$
\int_{\mathbb{R}^{4}} e^{4 u} d x<\infty
$$

and $|u(x)|=o\left(|x|^{2}\right)$ at $\infty$. Then there exists some point $x^{0} \in \mathbb{R}^{4}$ such that $u$ is radially symmetric about $x^{0}$ and

$$
u(x)=\ln \left(\frac{2 \lambda}{1+\lambda^{2}\left|x-x^{0}\right|^{2}}\right) .
$$

This result is decisive for solving completely 1.2 under 1.3 , because it reduces the problem to a simple ODE problem.

In [21], Wei and Ye constructed a non-radial solution of Liouville equation 1.2 under (1.3) with the following asymptotic behavior

$$
u(x)=-\sum_{j=1}^{k} a_{j}\left(x_{j}-x_{j}^{0}\right)^{2}-\alpha \ln |x|+c_{0}+o(1), \quad|x|>1 \quad \text { and } \quad \int_{\mathbb{R}^{4}} e^{4 u(x)} d x=\frac{4 \pi^{2} \alpha}{3}
$$

for each fixed $x^{0} \in \mathbb{R}^{4}, 1 \leq k \leq 4, \alpha \in(1-k / 4,2)$ and $a_{j}>0$ for $1 \leq j \leq k$.

We consider the corresponding Dirichlet problem on a bounded domain in $\mathbb{R}^{2}$,

$$
-\Delta u=\rho^{2} e^{u} \text { in } \Omega, \quad u=0 \text { on } \partial \Omega,
$$

where the parameter $\rho$ tends to 0 . The study of this equation goes back to 1853 when Liouville derived a representation formula for all solutions of $(1.4)$ that are defined in $\mathbb{R}^{2}[18]$.

It is well known that as the parameter $\rho$ tends to 0 , non-minimal solutions exist and they have singular limits. In [6], Baraket and Pacard proved

Theorem 1.2. [6] Let $\Omega$ be a smooth open subset of $\mathbb{R}^{2}$ and $z^{1}, \ldots, z^{m} \in \Omega$. Assume that $\left(z^{1}, \ldots, z^{m}\right)$ is a nondegenerate critical point of the function

$$
F:\left(z^{1}, \ldots, z^{m}\right) \in \mathbb{C}^{m} \rightarrow \sum_{j} h\left(z^{j}, z^{j}\right)+\sum_{j \neq l} g\left(z^{j}, z^{l}\right),
$$

then there exist $\rho_{0}>0$ and $\left(u_{\rho}\right)_{\rho \in\left(0, \rho_{0}\right)}$ a one parameter family of solutions of (1.4) such that

$$
\lim _{\rho \rightarrow 0} u_{\rho}=u^{*}:=\sum_{j=1}^{m} g\left(\cdot, z^{j}\right) \quad \text { in } \mathcal{C}_{\operatorname{loc}}^{2, \alpha}\left(\Omega-\left\{z^{1}, \ldots, z^{m}\right\}\right) .
$$

Here $g$ is the Green's function defined as the solution of

$$
\begin{cases}-\Delta_{z} g\left(z, z^{\prime}\right)=8 \pi \delta_{z=z^{\prime}} & \text { in } \Omega \\ g\left(z, z^{\prime}\right)=0 & \text { on } \partial \Omega\end{cases}
$$


and $h$ is its smooth part defined by $h\left(z, z^{\prime}\right)=g\left(z, z^{\prime}\right)+4 \ln \left|z-z^{\prime}\right|$. Some generalizations can be found in $3,8,14$.

In dimension 4 , other authors were motivated by similar problems, we refer the reader to [2, 4, 11, 12]. Wei in [20], has studied the behavior of solutions to the following nonlinear eigenvalue problem for the biharmonic operator $\Delta^{2}$ in $\mathbb{R}^{4}$. More precisely, consider the following problem

$$
\begin{cases}\Delta^{2} u=\lambda f(u) & \text { in } \Omega, \\ u=\Delta u=0 & \text { on } \partial \Omega .\end{cases}
$$

When $f(u)=e^{u}$, we can see that 1.5 is issued from the conformal geometry by prescribing the so-called $Q$-curvature on 4-dimensional Riemannian manifolds. For more details and background material we refer to $1,9,13]$.

Before announcing the result of [20], we will introduce some notations. Let $G\left(x, x^{\prime}\right)$ defined over $\Omega \times \Omega$, be the Green function associated to the bi-laplacian operator with Navier boundary conditions, which is the solution of

$$
\begin{cases}\Delta_{x}^{2} G\left(x, x^{\prime}\right)=64 \pi^{2} \delta_{x=x^{\prime}} & \text { in } \Omega \\ G\left(x, x^{\prime}\right)=\Delta_{x} G\left(x, x^{\prime}\right)=0 & \text { on } \partial \Omega\end{cases}
$$

and denote by $H\left(x, x^{\prime}\right)=G\left(x, x^{\prime}\right)+8 \ln \left|x-x^{\prime}\right|$ its smooth part. Consider now the functional

$$
E\left(x^{1}, \ldots, x^{m}\right)=\sum_{j=1}^{m} H\left(x^{j}, x^{j}\right)+\sum_{j \neq l} G\left(x^{j}, x^{l}\right)
$$

and denote by $u^{*}$ the solution of

$$
\begin{cases}\Delta^{2} u^{*}=64 \pi^{2} \sum_{j=1}^{m} \delta_{x^{j}} & \text { in } \Omega, \\ u^{*}=\Delta u^{*}=0 & \text { on } \partial \Omega .\end{cases}
$$

In [20], the author proved the following result.

Theorem 1.3. [20] Let $\Omega$ be a smooth bounded domain in $\mathbb{R}^{4}$ and $f$ a smooth nonnegative increasing function such that

$$
e^{-u} f(u) \text { tends to } 1 \text { as } u \rightarrow+\infty \text {. }
$$

For $u_{\lambda}$ solution of (1.5), denote by $\Sigma_{\lambda}=\lambda \int_{\Omega} f\left(u_{\lambda}\right) d x$. Then there are only three possibilities:

(i) The $\left\{\Sigma_{\lambda}\right\}$ accumulate to 0 . Then $\left\|u_{\lambda}\right\|_{L^{\infty}(\Omega)} \rightarrow 0$ as $\lambda \rightarrow 0$.

(ii) The $\left\{\Sigma_{\lambda}\right\}$ accumulate to $+\infty$. Then $u_{\lambda} \rightarrow+\infty$ as $\lambda \rightarrow 0$. 
(iii) The $\left\{\Sigma_{\lambda}\right\}$ accumulate to $64 \pi^{2} m$ for some positive integer $m$. Then the limiting function $u^{*}=\lim _{\lambda \rightarrow 0} u_{\lambda}$ has $m$ blow-up points, $\left\{x^{1}, \ldots, x^{m}\right\}$, where $u_{\lambda}\left(x^{i}\right) \rightarrow+\infty$ as $\lambda \rightarrow 0$.

Moreover, $\left(x^{1}, \ldots, x^{m}\right)$ is a critical point of $E$.

In dimension 4 , the authors in 5 considered the following problem

$$
\begin{cases}\Delta^{2} u_{1}=\rho^{4} e^{u_{1}+\gamma_{1} u_{2}} & \text { in } \Omega, \\ \Delta^{2} u_{2}=\rho^{4} e^{u_{2}+\gamma_{2} u_{1}} & \text { in } \Omega, \\ u_{i}=\Delta u_{i}=0, i=1,2 & \text { on } \partial \Omega .\end{cases}
$$

They proved the existence of singular limit solutions which blow-up on different points as $\rho$ tends to 0 using the nonlinear domain decomposition method.

In dimension 2 , the $L$-system has been interested by several authors $7,10,16,17,19]$. Recently, the authors in [7] considered the following problem

$$
\begin{cases}-\Delta u_{1}=\rho^{2} e^{\gamma u_{1}+(1-\gamma) u_{2}} & \text { in } \Omega, \\ -\Delta u_{2}=\rho^{2} e^{\xi u_{2}+(1-\xi) u_{1}} & \text { in } \Omega, \\ u_{1}=u_{2}=0 & \text { on } \partial \Omega .\end{cases}
$$

They proved the existence of singular limit solutions with blow-up on common points as $\rho$ tends to 0 .

In this paper, we prove the following results.

Theorem 1.4. Let $\Omega$ be a regular open subset of $\mathbb{R}^{4}$ and $x^{1}, x^{2}, x^{3} \in \Omega$ be given disjoint points. Suppose that $\left(u_{1}^{\rho}, u_{2}^{\rho}\right)$ is a one parameter family of solutions of (1.1), such that

$$
\lim _{\rho \rightarrow 0} u_{1}^{\rho}=\frac{1}{\gamma} G\left(\cdot, x^{1}\right)+G\left(\cdot, x^{2}\right)=u_{1}^{*} \quad \text { in } \mathcal{C}_{\text {loc }}^{4, \alpha}\left(\Omega \backslash\left\{x^{1}, x^{2}\right\}\right)
$$

and

$$
\lim _{\rho \rightarrow 0} u_{2}^{\rho}=\frac{1}{\xi} G\left(\cdot, x^{3}\right)+G\left(\cdot, x^{2}\right)=u_{2}^{*} \quad \text { in } \mathcal{C}_{\mathrm{loc}}^{4, \alpha}\left(\Omega \backslash\left\{x^{2}, x^{3}\right\}\right) .
$$

Then $\left(x^{1}, x^{2}, x^{3}\right)$ is a critical point of the functional

$$
\begin{aligned}
\mathcal{E}\left(x^{1}, x^{2}, x^{3}\right)= & \frac{1-\xi}{\gamma} H\left(x^{1}, x^{1}\right)+(2-\gamma-\xi) H\left(x^{2}, x^{2}\right)+\frac{1-\gamma}{\xi} H\left(x^{3}, x^{3}\right) \\
& +\frac{1-\gamma}{\xi} \frac{1-\xi}{\gamma} G\left(x^{1}, x^{3}\right)+\frac{1-\xi}{\gamma} G\left(x^{1}, x^{2}\right)+\frac{1-\gamma}{\xi} G\left(x^{2}, x^{3}\right) .
\end{aligned}
$$

A natural question that arises: can one find a solution that concentrates in a common point $x^{2}$. Before giving a partial answer of this question, we define an auxiliary function which is a cut-off function in $C_{0}^{\infty}(\Omega)$ such that $\varphi \equiv 1$ in $B\left(x^{1}, r_{0}\right) \cup B\left(x^{3}, r_{0}\right)$ and $\varphi \equiv 0$ in $\Omega \backslash\left(B\left(x^{1}, r_{0}\right) \cup B\left(x^{3}, r_{0}\right)\right)$, where $r_{0}>0$ and such that $B\left(x^{i}, 2 r_{0}\right) \subset \Omega$ for $i=1,3$ and $B\left(x^{1}, 2 r_{0}\right) \cap B\left(x^{3}, 2 r_{0}\right)=\emptyset$. 
Theorem 1.5. Let $\Omega$ be a regular open subset of $\mathbb{R}^{4}$ and $x^{1}, x^{2}, x^{3} \in \Omega$ be given disjoint points. Suppose that $\left(x^{1}, x^{2}, x^{3}\right)$ is a nondegenerate critical point of the functional

$$
\begin{aligned}
\mathcal{E}\left(x^{1}, x^{2}, x^{3}\right)= & \frac{1-\xi}{\gamma} H\left(x^{1}, x^{1}\right)+(2-\gamma-\xi) H\left(x^{2}, x^{2}\right)+\frac{1-\gamma}{\xi} H\left(x^{3}, x^{3}\right) \\
& +\frac{1-\gamma}{\xi} \frac{1-\xi}{\gamma} G\left(x^{1}, x^{3}\right)+\frac{1-\xi}{\gamma} G\left(x^{1}, x^{2}\right)+\frac{1-\gamma}{\xi} G\left(x^{2}, x^{3}\right) .
\end{aligned}
$$

Then there exist $\gamma_{0}$ and $\xi_{0}$ in $(0,1)$ such that for all $\gamma \in\left(\gamma_{0}, 1\right)$ and $\xi \in\left(\xi_{0}, 1\right)$, there exist $\rho_{0}>0$ and $\left(u_{1}^{\rho}, u_{2}^{\rho}\right)_{\rho \leq \rho_{0}}$ a one parameter family of solutions of (1.1), such that

$$
\lim _{\rho \rightarrow 0} \varphi u_{1}^{\rho}=\frac{\varphi}{\gamma} G\left(\cdot, x^{1}\right) \quad \text { in } \mathcal{C}_{\text {loc }}^{4, \alpha}\left(\Omega \backslash\left\{x^{1}\right\}\right), \quad \lim _{\rho \rightarrow 0} \varphi u_{2}^{\rho}=\frac{\varphi}{\xi} G\left(\cdot, x^{3}\right) \text { in } \mathcal{C}_{\text {loc }}^{4, \alpha}\left(\Omega \backslash\left\{x^{3}\right\}\right)
$$

and

$$
\begin{aligned}
& \lim _{\rho \rightarrow 0}\left((1-\xi) u_{1}^{\rho}+(1-\gamma) u_{2}^{\rho}\right) \\
= & \frac{1-\xi}{\gamma} G\left(\cdot, x^{1}\right)+\frac{1-\gamma}{\xi} G\left(\cdot, x^{3}\right)+(2-\gamma-\xi) G\left(\cdot, x^{2}\right) \text { in } \mathcal{C}_{\text {loc }}^{4, \alpha}\left(\Omega \backslash\left\{x^{1}, x^{2}, x^{3}\right\}\right) .
\end{aligned}
$$

Unfortunately, we are not able to give the asymptotic behavior of $u_{1}^{\rho}$ and $u_{2}^{\rho}$ separately. But, under an additional assumption on the points set $\left\{x^{1}, x^{2}, x^{3}\right\}$, we give a positive answer.

Theorem 1.6. Let $\Omega$ be a regular open subset of $\mathbb{R}^{4}$ and $x^{1}, x^{2}, x^{3} \in \Omega$ be given disjoint points. Suppose that $\left(x^{1}, x^{2}, x^{3}\right)$ is a nondegenerate critical point of the functional

$$
\begin{aligned}
\mathcal{E}\left(x^{1}, x^{2}, x^{3}\right)= & \frac{1-\xi}{\gamma} H\left(x^{1}, x^{1}\right)+(2-\gamma-\xi) H\left(x^{2}, x^{2}\right)+\frac{1-\gamma}{\xi} H\left(x^{3}, x^{3}\right) \\
& +\frac{1-\gamma}{\xi} \frac{1-\xi}{\gamma} G\left(x^{1}, x^{3}\right)+\frac{1-\xi}{\gamma} G\left(x^{1}, x^{2}\right)+\frac{1-\gamma}{\xi} G\left(x^{2}, x^{3}\right)
\end{aligned}
$$

such that

$$
\frac{1}{\gamma} G\left(x^{2}, x^{1}\right)=\frac{1}{\xi} G\left(x^{2}, x^{3}\right) \quad \text { and } \quad \frac{1}{\gamma} \nabla G\left(\cdot, x^{1}\right)\left(x^{2}\right)=\frac{1}{\xi} \nabla G\left(\cdot, x^{3}\right)\left(x^{2}\right) .
$$

Then there exist $\gamma_{0}$ and $\xi_{0}$ in $(0,1)$ such that for all $\gamma \in\left(\gamma_{0}, 1\right)$ and $\xi \in\left(\xi_{0}, 1\right)$, there exist $\rho_{0}>0$ and $\left(u_{1}^{\rho}, u_{2}^{\rho}\right)_{\rho \leq \rho_{0}}$ a one parameter family of solutions of 1.1 , such that

$$
\begin{aligned}
& \lim _{\rho \rightarrow 0} u_{1}^{\rho}=\frac{1}{\gamma} G\left(\cdot, x^{1}\right)+G\left(\cdot, x^{2}\right) \quad \text { in } \mathcal{C}_{\mathrm{loc}}^{4, \alpha}\left(\Omega \backslash\left\{x^{1}, x^{2}\right\}\right), \\
& \lim _{\rho \rightarrow 0} u_{2}^{\rho}=\frac{1}{\xi} G\left(\cdot, x^{3}\right)+G\left(\cdot, x^{2}\right) \quad \text { in } \mathcal{C}_{\mathrm{loc}}^{4, \alpha}\left(\Omega \backslash\left\{x^{2}, x^{3}\right\}\right) .
\end{aligned}
$$

Remark 1.7. Denote by $S_{i}$ the blow up points set of $u_{i}$ for $i=1,2$. 
1. Theorem 1.4 can be extended, but the computation is more complicated. Indeed we replace $\mathcal{E}\left(x^{1}, x^{2}, x^{3}\right)$ by $\mathcal{F}\left(x^{p} \in S_{1} \backslash S_{1} \cap S_{2}, x^{k} \in S_{1} \cap S_{2}, x^{l} \in S_{2} \backslash S_{1} \cap S_{2}\right)$, given by

$$
\begin{aligned}
& \mathcal{F}\left(x^{p} \in S_{1} \backslash S_{1} \cap S_{2}, x^{k} \in S_{1} \cap S_{2}, x^{l} \in S_{2} \backslash S_{1} \cap S_{2}\right) \\
& =\frac{1-\gamma}{\xi} \frac{1-\xi}{\gamma} \sum_{\substack{x^{l} \in S_{2} \backslash S_{1} \cap S_{2} \\
x^{p} \in S_{1} \backslash S_{1} \cap S_{2}}} G\left(x^{l}, x^{p}\right) \\
& +\frac{1-\xi}{\gamma}\left(\sum_{x^{p} \in S_{1} \backslash S_{1} \cap S_{2}} H\left(x^{p}, x^{p}\right)+\sum_{\substack{x^{k} \in S_{1} \cap S_{2} \\
x^{p} \in S_{1} \backslash S_{1} \cap S_{2}}} G\left(x^{k}, x^{p}\right)+\sum_{\substack{x^{p}, x^{p^{\prime}} \in S_{1} \backslash S_{1} \cap S_{2} \\
p \neq p^{\prime}}} G\left(x^{p}, x^{p^{\prime}}\right)\right) \\
& +\frac{1-\gamma}{\xi}\left(\sum_{x^{l} \in S_{2} \backslash S_{1} \cap S_{2}} H\left(x^{l}, x^{l}\right)+\sum_{\substack{x^{l} \in S_{2} \backslash S_{1} \cap S_{2} \\
x^{k} \in S_{1} \cap S_{2}}} G\left(x^{l}, x^{k}\right)+\sum_{\substack{x^{l}, x^{l^{\prime}} \in S_{2} \backslash S_{1} \cap S_{2} \\
l \neq l^{\prime}}} G\left(x^{l}, x^{l^{\prime}}\right)\right) \\
& +(2-\gamma-\xi)\left(\sum_{x^{k} \in S_{1} \cap S_{2}} H\left(x^{k}, x^{k}\right)+\sum_{x^{k}, x^{k^{\prime}} \in S_{1} \cap S_{2}} G\left(x^{k}, x^{k^{\prime}}\right)\right) .
\end{aligned}
$$

2. Theorem 1.5 can be also extended by assuming that $\left(x^{p} \in S_{1} \backslash S_{1} \cap S_{2}, x^{k} \in S_{1} \cap\right.$ $\left.S_{2}, x^{l} \in S_{2} \backslash S_{1} \cap S_{2}\right)$ is a nondegenerate critical point of $\mathcal{F}$.

3. Theorem 1.6 is hold if $S_{1} \backslash S_{1} \cap S_{2} \neq \emptyset$ and $S_{2} \backslash S_{1} \cap S_{2} \neq \emptyset$ and the condition (1.6) will be replaced for all $x^{k} \in S_{1} \cap S_{2}$ by

$$
\frac{1}{\gamma} \sum_{x^{p} \in S_{1} \backslash S_{1} \cap S_{2}} G\left(x^{k}, x^{p}\right)=\frac{1}{\xi} \sum_{x^{l} \in S_{2} \backslash S_{1} \cap S_{2}} G\left(x^{k}, x^{l}\right)
$$

and

$$
\frac{1}{\gamma} \sum_{x^{p} \in S_{1} \backslash S_{1} \cap S_{2}} \nabla G\left(\cdot, x^{p}\right)\left(x^{k}\right)=\frac{1}{\xi} \sum_{x^{l} \in S_{2} \backslash S_{1} \cap S_{2}} \nabla G\left(\cdot, x^{l}\right)\left(x^{k}\right) .
$$

4. To have a readable and clear proof, we considered the choice of $S_{1}=\left\{x^{1}, x^{2}\right\}$ and $S_{2}=\left\{x^{2}, x^{3}\right\}$, which is already supposed in Theorems 1.4, 1.5 and 1.6 .

5. Our functional presents the same terms as those of the functional introduced by Baraket-Pacard [6], the only difference here is that these terms are weighted by certain weights not equals.

6. The conditions of Theorem 1.6 are certainly not valid on all domain $\Omega$ of $\mathbb{R}^{4}$. It is thought that a certain symmetry of the domain must be imposed for the condition (1.6) to be verified.

\section{Proof of Theorem 1.4}

We first give the green identity for the bilaplacian operator:

$$
\int_{\Omega}\left(\Delta^{2} u\right) \cdot v-\left(\Delta^{2} v\right) \cdot u=\int_{\partial \Omega}\left(\frac{\partial \Delta u}{\partial \nu} \cdot v-\Delta u \frac{\partial v}{\partial \nu}+\frac{\partial u}{\partial \nu} \cdot \Delta v-u \cdot \frac{\partial \Delta v}{\partial \nu}\right) d \sigma .
$$


2.1. Behavior of solution around $x^{2}$

We multiply the equation $\Delta^{2} u_{1}=\rho^{4} e^{\gamma u_{1}+(1-\gamma) u_{2}}$ by $\nabla\left(\gamma u_{1}+(1-\gamma) u_{2}\right)$ and then integrating over $B_{2}=B\left(x^{2}, \eta\right)$ where $\eta$ fixed small enough, we obtain a pohozaev type identity

$$
\gamma \int_{B_{2}}\left(\Delta^{2} u_{1}\right) \nabla u_{1}+(1-\gamma) \int_{B_{2}}\left(\Delta^{2} u_{1}\right) \nabla u_{2}=\rho^{4} \int_{\partial B_{2}}\left(e^{\gamma u_{1}+(1-\gamma) u_{2}}-1\right) \nu d \sigma
$$

Using the Green's formula we obtain

$$
\begin{aligned}
\int_{B_{2}}\left(\Delta^{2} u_{1}\right) \nabla u_{1}= & -\int_{B_{2}}\left(\Delta u_{1}\right) \nabla\left(\Delta\left(u_{1}\right)\right)-\int_{B_{2}} \nabla\left(\nabla\left(\Delta u_{1}\right) \cdot \nabla u_{1}\right) \\
& +\int_{\partial B_{2}} \nabla\left(\Delta u_{1}\right) \cdot \nu \nabla u_{1} d \sigma+\int_{\partial B_{2}} \nabla u_{1} \cdot \nu \nabla\left(\Delta u_{1}\right) d \sigma \\
= & -\frac{1}{2} \int_{\partial B_{2}}\left(\Delta u_{1}\right)^{2} \nu d \sigma-\int_{\partial B_{2}}\left(\nabla\left(\Delta u_{1}\right) \cdot \nabla u_{1}\right) \nu d \sigma \\
& +\int_{\partial B_{2}} \nabla\left(\Delta u_{1}\right) \cdot \nu \nabla u_{1} d \sigma+\int_{\partial B_{2}} \nabla u_{1} \cdot \nu \nabla\left(\Delta u_{1}\right) d \sigma
\end{aligned}
$$

Similarly we multiply the equation $\Delta^{2} u_{2}=\rho^{4} e^{\xi u_{2}+(1-\xi) u_{1}}$ by $\nabla\left(\xi u_{2}+(1-\xi) u_{1}\right)$ and then integrating over $B_{2}=B\left(x^{2}, \eta\right)$ we obtain a pohozaev type identity

$$
\xi \int_{B_{2}}\left(\Delta^{2} u_{2}\right) \nabla u_{2}+(1-\xi) \int_{B_{2}}\left(\Delta^{2} u_{2}\right) \nabla u_{1}=\rho^{4} \int_{\partial B_{2}}\left(e^{\xi u_{2}+(1-\xi) u_{1}}-1\right) \nu d \sigma .
$$

Using the Green's formula we obtain

$$
\begin{aligned}
\int_{B_{2}}\left(\Delta^{2} u_{2}\right) \nabla u_{2}= & -\int_{B_{2}}\left(\Delta u_{2}\right) \nabla\left(\Delta\left(u_{2}\right)\right)-\int_{B_{2}} \nabla\left(\nabla\left(\Delta u_{2}\right) \cdot \nabla u_{2}\right) \\
& +\int_{\partial B_{2}} \nabla\left(\Delta u_{2}\right) \cdot \nu \nabla u_{2} d \sigma+\int_{\partial B_{2}} \nabla u_{2} \cdot \nu \nabla\left(\Delta u_{2}\right) d \sigma \\
= & -\frac{1}{2} \int_{\partial B_{2}}\left(\Delta u_{2}\right)^{2} \nu d \sigma-\int_{\partial B_{2}}\left(\nabla\left(\Delta u_{2}\right) \cdot \nabla u_{2}\right) \nu d \sigma \\
& +\int_{\partial B_{2}} \nabla\left(\Delta u_{2}\right) \cdot \nu \nabla u_{2} d \sigma+\int_{\partial B_{2}} \nabla u_{2} \cdot \nu \nabla\left(\Delta u_{2}\right) d \sigma
\end{aligned}
$$

Making use of the identity

$$
\begin{aligned}
& \int_{B_{2}} \Delta^{2} u_{2} \nabla u_{1}+\int_{B_{2}} \Delta^{2} u_{1} \nabla u_{2} \\
= & -\int_{B_{2}} \nabla\left(\Delta u_{2} \cdot \Delta u_{1}\right)+\int_{\partial B_{2}} \frac{\partial\left(\Delta u_{2}\right)}{\partial \nu} \nabla u_{1} d \sigma+\int_{\partial B_{2}} \frac{\partial\left(\Delta u_{1}\right)}{\partial \nu} \nabla u_{2} d \sigma \\
= & -\int_{\partial B_{2}}\left(\Delta u_{2} \cdot \Delta u_{1}\right) \nu d \sigma+\int_{\partial B_{2}} \frac{\partial\left(\Delta u_{2}\right)}{\partial \nu} \nabla u_{1} d \sigma+\int_{\partial B_{2}} \frac{\partial\left(\Delta u_{1}\right)}{\partial \nu} \nabla u_{2} d \sigma,
\end{aligned}
$$


then by combination of 2.1 and 2.2 we obtain

$$
\begin{aligned}
& \gamma(1-\xi) \int_{\partial B_{2}}\left[\frac{-1}{2}\left(\Delta u_{1}\right)^{2} \nu-\left(\nabla\left(\Delta u_{1}\right) \cdot \nabla u_{1}\right) \nu+\nabla\left(\Delta u_{1}\right) \cdot \nu \nabla u_{1}+\nabla u_{1} \cdot \nu \nabla\left(\Delta u_{1}\right)\right] d \sigma \\
& +\xi(1-\gamma) \int_{\partial B_{2}}\left[\frac{-1}{2}\left(\Delta u_{2}\right)^{2} \nu-\left(\nabla\left(\Delta u_{2}\right) \cdot \nabla u_{2}\right) \nu+\nabla\left(\Delta u_{2}\right) \cdot \nu \nabla u_{2}+\nabla u_{2} \cdot \nu \nabla\left(\Delta u_{2}\right)\right] d \sigma \\
& +(1-\gamma)(1-\xi)\left[-\int_{\partial B_{2}}\left(\Delta u_{2} \cdot \Delta u_{1}\right) \nu d \sigma+\int_{\partial B_{2}} \frac{\partial\left(\Delta u_{2}\right)}{\partial \nu} \nabla u_{1} d \sigma+\int_{\partial B_{2}} \frac{\partial\left(\Delta u_{1}\right)}{\partial \nu} \nabla u_{2} d \sigma\right] \\
& =\rho^{4}(1-\xi) \int_{\partial B_{2}}\left(e^{\gamma u_{1}+(1-\gamma) u_{2}}-1\right) \nu d \sigma+\rho^{4}(1-\gamma) \int_{\partial B_{2}}\left(e^{\xi u_{2}+(1-\xi) u_{1}}-1\right) \nu d \sigma .
\end{aligned}
$$

In the desire to construct solutions of the system that blow-up in the point $x^{2}$ this means that, if $\rho$ tends to zero,

$$
u_{1} \rightarrow u_{1}^{*}(x)=G\left(x, x^{2}\right)+\frac{1}{\gamma} G\left(x, x^{1}\right) \quad \text { and } \quad u_{2} \rightarrow u_{2}^{*}(x)=G\left(x, x^{2}\right)+\frac{1}{\xi} G\left(x, x^{3}\right) .
$$

Since we have $G\left(x, x^{2}\right)=-8 \ln \left|x-x^{2}\right|+H\left(x, x^{2}\right)$, where $H$ is a smooth function in $\Omega$, then

$$
\begin{aligned}
u_{1}^{*}(x) & =G\left(x, x^{2}\right)+\frac{1}{\gamma} G\left(x, x^{1}\right)=-8 \ln \left|x-x^{2}\right|+H\left(x, x^{2}\right)+\frac{1}{\gamma} G\left(x, x^{1}\right) \\
& =-8 \ln \left|x-x^{2}\right|+R\left(x, x^{2}\right)
\end{aligned}
$$

and

$$
\begin{aligned}
u_{2}^{*}(x) & =G\left(x, x^{2}\right)+\frac{1}{\xi} G\left(x, x^{3}\right)=-8 \ln \left|x-x^{2}\right|+H\left(x, x^{2}\right)+\frac{1}{\xi} G\left(x, x^{3}\right) \\
& =-8 \ln \left|x-x^{2}\right|+K\left(x, x^{2}\right) .
\end{aligned}
$$

Thanks to the fact that the solutions of the system (1.1) are regular on $\Omega \backslash\left\{x^{1}, x^{2}, x^{3}\right\}$ and by inserting the profile of the limits of the solutions in the identity 2.3 when $\rho \rightarrow 0$ and $\eta$ fixed small enough, we obtain

$$
\lim _{\rho \rightarrow 0} \rho^{4}(1-\xi) \int_{\partial B_{2}}\left(e^{\gamma u_{1}+(1-\gamma) u_{2}}-1\right) \nu d \sigma+\rho^{4}(1-\gamma) \int_{\partial B_{2}}\left(e^{\xi u_{2}+(1-\xi) u_{1}}-1\right) \nu d \sigma=0
$$

then

$$
\begin{aligned}
& \gamma(1-\xi) \int_{\partial B_{2}}\left[\frac{-1}{2}\left(\Delta u_{1}^{*}\right)^{2} \nu-\left(\nabla\left(\Delta u_{1}^{*}\right) \cdot \nabla u_{1}^{*}\right) \nu+\nabla\left(\Delta u_{1}^{*}\right) \cdot \nu \nabla u_{1}^{*}+\nabla u_{1}^{*} \cdot \nu \nabla\left(\Delta u_{1}^{*}\right)\right] d \sigma \\
+ & \xi(1-\gamma) \int_{\partial B_{2}}\left[\frac{-1}{2}\left(\Delta u_{2}^{*}\right)^{2} \nu-\left(\nabla\left(\Delta u_{2}^{*}\right) \cdot \nabla u_{2}^{*}\right) \nu+\nabla\left(\Delta u_{2}^{*}\right) \cdot \nu \nabla u_{2}^{*}+\nabla u_{2}^{*} \cdot \nu \nabla\left(\Delta u_{2}^{*}\right)\right] d \sigma \\
+ & (1-\gamma)(1-\xi)\left[-\int_{\partial B_{2}}\left(\Delta u_{2}^{*} \cdot \Delta u_{1}^{*}\right) \nu d \sigma+\int_{\partial B_{2}} \frac{\partial\left(\Delta u_{2}^{*}\right)}{\partial \nu} \nabla u_{1}^{*} d \sigma+\int_{\partial B_{2}} \frac{\partial\left(\Delta u_{1}^{*}\right)}{\partial \nu} \nabla u_{2}^{*} d \sigma\right]=0 .
\end{aligned}
$$


We set

$$
\begin{aligned}
I_{l h s}= & \gamma(1-\xi) \int_{\partial B_{2}}\left[\frac{-1}{2}\left(\Delta u_{1}^{*}\right)^{2} \nu-\left(\nabla\left(\Delta u_{1}^{*}\right) \cdot \nabla u_{1}^{*}\right) \nu+\nabla\left(\Delta u_{1}^{*}\right) \cdot \nu \nabla u_{1}^{*}+\nabla u_{1}^{*} \cdot \nu \nabla\left(\Delta u_{1}^{*}\right)\right] d \sigma \\
& +\xi(1-\gamma) \int_{\partial B_{2}}\left[\frac{-1}{2}\left(\Delta u_{2}^{*}\right)^{2} \nu-\left(\nabla\left(\Delta u_{2}^{*}\right) \cdot \nabla u_{2}^{*}\right) \nu+\nabla\left(\Delta u_{2}^{*}\right) \cdot \nu \nabla u_{2}^{*}+\nabla u_{2}^{*} \cdot \nu \nabla\left(\Delta u_{2}^{*}\right)\right] d \sigma \\
& +(1-\gamma)(1-\xi)\left[-\int_{\partial B_{2}}\left(\Delta u_{2}^{*} \cdot \Delta u_{1}^{*}\right) \nu d \sigma+\int_{\partial B_{2}} \frac{\partial\left(\Delta u_{2}^{*}\right)}{\partial \nu} \nabla u_{1}^{*} d \sigma+\int_{\partial B_{2}} \frac{\partial\left(\Delta u_{1}^{*}\right)}{\partial \nu} \nabla u_{2}^{*} d \sigma\right],
\end{aligned}
$$

by computation, we prove that

$$
\begin{aligned}
I_{l h s}= & -\frac{8}{\eta} \gamma(1-\xi) \int_{\partial B_{2}} \nabla \Delta R\left(x, x^{2}\right) d \sigma-\frac{8}{\eta} \xi(1-\gamma) \int_{\partial B_{2}} \nabla \Delta K\left(x, x^{2}\right) d \sigma \\
& +\frac{8}{\eta}(1-\xi)(1-\gamma) \int_{\partial B_{2}}\left(\left(\nabla \Delta K\left(x, x^{2}\right)+\nabla \Delta R\left(x, x^{2}\right)\right) \cdot \nu\right) \nu d \sigma \\
& +\frac{16}{\eta^{2}}\left[(1-\xi) \int_{\partial B_{2}} \Delta R\left(x, x^{2}\right) \nu d \sigma+(1-\gamma) \int_{\partial B_{2}} \Delta K\left(x, x^{2}\right) \nu d \sigma\right] \\
& +\frac{32}{\eta^{3}}\left[(1-\xi) \int_{\partial B_{2}} \nabla R\left(x, x^{2}\right) d \sigma+(1-\gamma) \int_{\partial B_{2}} \nabla K\left(x, x^{2}\right) d \sigma\right]+O(\eta) .
\end{aligned}
$$

Then we have

$$
\begin{aligned}
& -8 \eta \gamma(1-\xi) \int_{\partial B_{2}} \nabla \Delta R\left(x, x^{2}\right) d \sigma-8 \eta \xi(1-\gamma) \int_{\partial B_{2}} \nabla \Delta K\left(x, x^{2}\right) d \sigma \\
& +8 \eta(1-\xi)(1-\gamma) \int_{\partial B_{2}}\left(\left(\nabla \Delta K\left(x, x^{2}\right)+\nabla \Delta R\left(x, x^{2}\right)\right) \cdot \nu\right) \nu d \sigma \\
& +16\left[(1-\xi) \int_{\partial B_{2}} \Delta R\left(x, x^{2}\right) \nu d \sigma+(1-\gamma) \int_{\partial B_{2}} \Delta K\left(x, x^{2}\right) \nu d \sigma\right] \\
& +\frac{32}{\eta}\left[(1-\xi) \int_{\partial B_{2}} \nabla R\left(x, x^{2}\right) d \sigma+(1-\gamma) \int_{\partial B_{2}} \nabla K\left(x, x^{2}\right) d \sigma\right]=O\left(\eta^{3}\right) .
\end{aligned}
$$

Writing $\nabla R\left(x, x^{2}\right)=\nabla R\left(x^{2}, x^{2}\right)+O(\eta)$ and $\nabla K\left(x, x^{2}\right)=\nabla K\left(x^{2}, x^{2}\right)+O(\eta)$ we obtain

$$
(1-\xi) \nabla R\left(x^{2}, x^{2}\right)+(1-\gamma) \nabla K\left(x^{2}, x^{2}\right)=O\left(\eta^{3}\right),
$$

which means that $x^{2}$ is a critical point of the functional

$$
\mathcal{E}_{2}: x \longmapsto \frac{1-\xi}{\gamma(2-\gamma-\xi)} G\left(x, x^{1}\right)+H\left(x, x^{2}\right)+\frac{1-\gamma}{\xi(2-\gamma-\xi)} G\left(x, x^{3}\right)
$$

2.2. Behavior of solution around $x^{1}$ and $x^{3}$

We multiply the equation $\Delta^{2} u_{1}=\rho^{4} e^{\gamma u_{1}+(1-\gamma) u_{2}}$ by $\nabla\left(\gamma u_{1}+(1-\gamma) u_{2}\right)$ and then integrating over $B_{1}=B\left(x^{1}, \eta\right)$ we obtain a Pohozaev type identity

$$
\gamma \int_{B_{1}}\left(\Delta^{2} u_{1}\right) \nabla u_{1}+(1-\gamma) \int_{B_{1}}\left(\Delta^{2} u_{1}\right) \nabla u_{2}=\rho^{4} \int_{\partial B_{1}}\left(e^{\gamma u_{1}+(1-\gamma) u_{2}}-1\right) \nu d \sigma
$$


Using the Green's formula we obtain

$$
\begin{aligned}
\int_{B_{1}}\left(\Delta^{2} u_{1}\right) \nabla u_{1}= & -\int_{B_{1}}\left(\Delta u_{1}\right) \nabla\left(\Delta\left(u_{1}\right)\right)-\int_{B_{1}} \nabla\left(\nabla\left(\Delta u_{1}\right) \cdot \nabla u_{1}\right) \\
& +\int_{\partial B_{1}} \nabla\left(\Delta u_{1}\right) \cdot \nu \nabla u_{1} d \sigma+\int_{\partial B_{1}} \nabla u_{1} \cdot \nu \nabla\left(\Delta u_{1}\right) d \sigma \\
= & -\frac{1}{2} \int_{\partial B_{1}}\left(\Delta u_{1}\right)^{2} \nu d \sigma-\int_{\partial B_{1}}\left(\nabla\left(\Delta u_{1}\right) \cdot \nabla u_{1}\right) \nu d \sigma \\
& +\int_{\partial B_{1}} \nabla\left(\Delta u_{1}\right) \cdot \nu \nabla u_{1} d \sigma+\int_{\partial B_{1}} \nabla u_{1} \cdot \nu \nabla\left(\Delta u_{1}\right) d \sigma
\end{aligned}
$$

Similarly we multiply the equation $\Delta^{2} u_{2}=\rho^{4} e^{\xi u_{2}+(1-\xi) u_{1}}$ by $\nabla\left(\xi u_{2}+(1-\xi) u_{1}\right)$ and then integrating over $B_{1}=B\left(x^{1}, \eta\right)$ we obtain a Pohozaev type identity

$$
\xi \int_{B_{1}}\left(\Delta^{2} u_{2}\right) \nabla u_{2}+(1-\xi) \int_{B_{1}}\left(\Delta^{2} u_{2}\right) \nabla u_{1}=\rho^{4} \int_{\partial B_{1}}\left(e^{\xi u_{2}+(1-\xi) u_{1}}-1\right) \nu d \sigma .
$$

Using the Green's formula we obtain

$$
\begin{aligned}
\int_{B_{1}}\left(\Delta^{2} u_{2}\right) \nabla u_{2}= & -\int_{B_{1}}\left(\Delta u_{2}\right) \nabla\left(\Delta\left(u_{2}\right)\right)-\int_{B_{1}} \nabla\left(\nabla\left(\Delta u_{2}\right) \cdot \nabla u_{2}\right) \\
& +\int_{\partial B_{1}} \nabla\left(\Delta u_{2}\right) \cdot \nu \nabla u_{2} d \sigma+\int_{\partial B_{1}} \nabla u_{2} \cdot \nu \nabla\left(\Delta u_{2}\right) d \sigma \\
= & -\frac{1}{2} \int_{\partial B_{1}}\left(\Delta u_{2}\right)^{2} \nu d \sigma-\int_{\partial B_{1}}\left(\nabla\left(\Delta u_{2}\right) \cdot \nabla u_{2}\right) \nu d \sigma \\
& +\int_{\partial B_{1}} \nabla\left(\Delta u_{2}\right) \cdot \nu \nabla u_{2} d \sigma+\int_{\partial B_{1}} \nabla u_{2} \cdot \nu \nabla\left(\Delta u_{2}\right) d \sigma
\end{aligned}
$$

Making use of the identity

$$
\begin{aligned}
& \int_{B_{1}} \Delta^{2} u_{2} \nabla u_{1}+\int_{B_{1}} \Delta^{2} u_{1} \nabla u_{2} \\
= & -\int_{B_{1}} \nabla\left(\Delta u_{2} \cdot \Delta u_{1}\right)+\int_{\partial B_{1}} \frac{\partial\left(\Delta u_{2}\right)}{\partial \nu} \nabla u_{1} d \sigma+\int_{\partial B_{1}} \frac{\partial\left(\Delta u_{1}\right)}{\partial \nu} \nabla u_{2} d \sigma \\
= & -\int_{\partial B_{1}}\left(\Delta u_{2} \cdot \Delta u_{1}\right) \nu d \sigma+\int_{\partial B_{1}} \frac{\partial\left(\Delta u_{2}\right)}{\partial \nu} \nabla u_{1} d \sigma+\int_{\partial B_{1}} \frac{\partial\left(\Delta u_{1}\right)}{\partial \nu} \nabla u_{2} d \sigma,
\end{aligned}
$$

then by combination of (2.5) and (2.6) we obtain

$$
\begin{aligned}
& \gamma(1-\xi) \int_{\partial B_{1}}\left[\frac{-1}{2}\left(\Delta u_{1}\right)^{2} \nu-\left(\nabla\left(\Delta u_{1}\right) \cdot \nabla u_{1}\right) \nu+\nabla\left(\Delta u_{1}\right) \cdot \nu \nabla u_{1}+\nabla u_{1} \cdot \nu \nabla\left(\Delta u_{1}\right)\right] d \sigma \\
& +\xi(1-\gamma) \int_{\partial B_{1}}\left[\frac{-1}{2}\left(\Delta u_{2}\right)^{2} \nu-\left(\nabla\left(\Delta u_{2}\right) \cdot \nabla u_{2}\right) \nu+\nabla\left(\Delta u_{2}\right) \cdot \nu \nabla u_{2}+\nabla u_{2} \cdot \nu \nabla\left(\Delta u_{2}\right)\right] d \sigma \\
& +(1-\gamma)(1-\xi)\left[-\int_{\partial B_{1}}\left(\Delta u_{2} \cdot \Delta u_{1}\right) \nu d \sigma+\int_{\partial B_{1}} \frac{\partial\left(\Delta u_{2}\right)}{\partial \nu} \nabla u_{1} d \sigma+\int_{\partial B_{1}} \frac{\partial\left(\Delta u_{1}\right)}{\partial \nu} \nabla u_{2} d \sigma\right] \\
& =\rho^{4}(1-\xi) \int_{\partial B_{1}}\left(e^{\gamma u_{1}+(1-\gamma) u_{2}}-1\right) \nu d \sigma+\rho^{4}(1-\gamma) \int_{\partial B_{1}}\left(e^{\xi u_{2}+(1-\xi) u_{1}}-1\right) \nu d \sigma .
\end{aligned}
$$


In the desire to construct solutions of the system that blow-up at the point $x^{1}$ this means that, if $\rho$ tends to zero,

$$
u_{1} \rightarrow u_{1}^{*}(x)=G\left(x, x^{2}\right)+\frac{1}{\gamma} G\left(x, x^{1}\right) \quad \text { and } \quad u_{2} \rightarrow u_{2}^{*}(x)=G\left(x, x^{2}\right)+\frac{1}{\xi} G\left(x, x^{3}\right) .
$$

Since we have $G\left(x, x^{1}\right)=-8 \ln \left|x-x^{1}\right|+H\left(x, x^{1}\right)$, where $H$ is a smooth function in $\Omega$, then

$$
\begin{aligned}
u_{1}^{*}(x) & =G\left(x, x^{2}\right)+\frac{1}{\gamma} G\left(x, x^{1}\right)=-\frac{8}{\gamma} \ln \left|x-x^{1}\right|+\frac{1}{\gamma} H\left(x, x^{1}\right)+G\left(x, x^{2}\right) \\
& =-\frac{8}{\gamma} \ln \left|x-x^{1}\right|+S\left(x, x^{1}\right)
\end{aligned}
$$

and

$$
u_{2}^{*}(x)=G\left(x, x^{2}\right)+\frac{1}{\xi} G\left(x, x^{3}\right)=T\left(x, x^{2}, x^{3}\right) .
$$

Thanks to the fact that the solutions of the system (1.1) are regular on $\Omega \backslash\left\{x^{1}, x^{2}, x^{3}\right\}$ and by inserting the profile of the limits of the solutions in the identity 2.3 when $\rho \rightarrow 0$ and $\eta$ fixed small enough, we obtain

$$
\lim _{\rho \rightarrow 0} \rho^{4}(1-\xi) \int_{\partial B_{1}}\left(e^{\gamma u_{1}+(1-\gamma) u_{2}}-1\right) \nu d \sigma+\rho^{4}(1-\gamma) \int_{\partial B_{2}}\left(e^{\xi u_{2}+(1-\xi) u_{1}}-1\right) \nu d \sigma=0,
$$

then

$$
\begin{aligned}
& \gamma(1-\xi) \int_{\partial B_{1}}\left[\frac{-1}{2}\left(\Delta u_{1}^{*}\right)^{2} \nu-\left(\nabla\left(\Delta u_{1}^{*}\right) \cdot \nabla u_{1}^{*}\right) \nu+\nabla\left(\Delta u_{1}^{*}\right) \cdot \nu \nabla u_{1}^{*}+\nabla u_{1}^{*} \cdot \nu \nabla\left(\Delta u_{1}^{*}\right)\right] d \sigma \\
+ & \xi(1-\gamma) \int_{\partial B_{1}}\left[\frac{-1}{2}\left(\Delta u_{2}^{*}\right)^{2} \nu-\left(\nabla\left(\Delta u_{2}^{*}\right) \cdot \nabla u_{2}^{*}\right) \nu+\nabla\left(\Delta u_{2}^{*}\right) \cdot \nu \nabla u_{2}^{*}+\nabla u_{2}^{*} \cdot \nu \nabla\left(\Delta u_{2}^{*}\right)\right] d \sigma \\
+ & (1-\gamma)(1-\xi)\left[-\int_{\partial B_{1}}\left(\Delta u_{2}^{*} \cdot \Delta u_{1}^{*}\right) \nu d \sigma+\int_{\partial B_{1}} \frac{\partial\left(\Delta u_{2}^{*}\right)}{\partial \nu} \nabla u_{1}^{*} d \sigma+\int_{\partial B_{1}} \frac{\partial\left(\Delta u_{1}^{*}\right)}{\partial \nu} \nabla u_{2}^{*} d \sigma\right]=0 .
\end{aligned}
$$

We set

$$
\begin{aligned}
I_{l h s}= & \gamma(1-\xi) \int_{\partial B_{1}}\left[\frac{-1}{2}\left(\Delta u_{1}^{*}\right)^{2} \nu-\left(\nabla\left(\Delta u_{1}^{*}\right) \cdot \nabla u_{1}^{*}\right) \nu+\nabla\left(\Delta u_{1}^{*}\right) \cdot \nu \nabla u_{1}^{*}+\nabla u_{1}^{*} \cdot \nu \nabla\left(\Delta u_{1}^{*}\right)\right] d \sigma \\
& +\xi(1-\gamma) \int_{\partial B_{1}}\left[\frac{-1}{2}\left(\Delta u_{2}^{*}\right)^{2} \nu-\left(\nabla\left(\Delta u_{2}^{*}\right) \cdot \nabla u_{2}^{*}\right) \nu+\nabla\left(\Delta u_{2}^{*}\right) \cdot \nu \nabla u_{2}^{*}+\nabla u_{2}^{*} \cdot \nu \nabla\left(\Delta u_{2}^{*}\right)\right] d \sigma \\
& +(1-\gamma)(1-\xi)\left[-\int_{\partial B_{1}}\left(\Delta u_{2}^{*} \cdot \Delta u_{1}^{*}\right) \nu d \sigma+\int_{\partial B_{1}} \frac{\partial\left(\Delta u_{2}^{*}\right)}{\partial \nu} \nabla u_{1}^{*} d \sigma+\int_{\partial B_{1}} \frac{\partial\left(\Delta u_{1}^{*}\right)}{\partial \nu} \nabla u_{2}^{*} d \sigma\right],
\end{aligned}
$$

by computation, we prove that

$$
\begin{aligned}
I_{l h s}= & -\frac{8}{\eta}(1-\xi) \int_{\partial B_{1}} \nabla \Delta S\left(x, x^{1}\right) d \sigma+\frac{8}{\gamma \eta}(1-\gamma)(1-\xi) \int_{\partial B_{1}}\left(\frac{\partial \Delta T\left(x, x^{2}, x^{3}\right)}{\partial \nu}\right) \nu d \sigma \\
& +\frac{16}{\eta^{2}}\left[(1-\xi) \int_{\partial B_{1}} \Delta S\left(x, x^{1}\right) \nu d \sigma+\frac{(1-\gamma)(1-\xi)}{\gamma} \int_{\partial B_{1}} \Delta T\left(x, x^{2}, x^{3}\right) \nu d \sigma\right] \\
& +\frac{32}{\eta^{3}}\left[(1-\xi) \int_{\partial B_{1}} \nabla S\left(x, x^{1}\right) d \sigma+\frac{(1-\gamma)(1-\xi)}{\gamma} \int_{\partial B_{1}} \nabla T\left(x, x^{2}, x^{3}\right) d \sigma\right]+O(\eta) .
\end{aligned}
$$


Then we have

$$
\begin{aligned}
& -8 \eta(1-\xi) \int_{\partial B_{1}} \nabla \Delta S\left(x, x^{1}\right) d \sigma+\frac{8 \eta}{\gamma}(1-\gamma)(1-\xi) \int_{\partial B_{1}}\left(\frac{\partial \Delta T\left(x, x^{2}, x^{3}\right)}{\partial \nu}\right) \nu d \sigma \\
& +16\left[(1-\xi) \int_{\partial B_{1}} \Delta S\left(x, x^{1}\right) \nu d \sigma+\frac{(1-\gamma)(1-\xi)}{\gamma} \int_{\partial B_{1}} \Delta T\left(x, x^{2}, x^{3}\right) \nu d \sigma\right] \\
& +\frac{32}{\eta}\left[(1-\xi) \int_{\partial B_{1}} \nabla S\left(x, x^{1}\right) d \sigma+\frac{(1-\gamma)(1-\xi)}{\gamma} \int_{\partial B_{1}} \nabla T\left(x, x^{2}, x^{3}\right) d \sigma\right]=O\left(\eta^{3}\right) .
\end{aligned}
$$

Writing $\nabla S\left(x, x^{1}\right)=\nabla S\left(x^{1}, x^{1}\right)+O(\eta)$ and $\nabla T\left(x, x^{2}, x^{3}\right)=\nabla T\left(x^{1}, x^{2}, x^{3}\right)+O(\eta)$ we obtain

$$
(1-\xi) \nabla S\left(x^{1}, x^{1}\right)+\frac{(1-\gamma)(1-\xi)}{\gamma} \nabla T\left(x^{1}, x^{2}, x^{3}\right)=O\left(\eta^{3}\right)
$$

which means that $x^{1}$ is a critical point of the functional

$$
\mathcal{E}_{1}: x \longmapsto H\left(\cdot, x^{1}\right)+G\left(\cdot, x^{2}\right)+\frac{1-\gamma}{\xi} G\left(\cdot, x^{3}\right)
$$

In $B_{3}=B\left(x^{3}, \eta\right)$, we proceed similarly as in $B_{1}=B\left(x^{1}, \eta\right)$ and respect the changes we obtain that $x^{3}$ is a critical point of the functional

$$
\mathcal{E}_{3}: x \longmapsto H\left(\cdot, x^{3}\right)+G\left(\cdot, x^{2}\right)+\frac{1-\xi}{\gamma} G\left(\cdot, x^{1}\right) .
$$

Finally by combination of (2.4), 2.7) and (2.8) we conclude that the point $\left(x^{1}, x^{2}, x^{3}\right)$ is a critical point of the functional $\mathcal{E}$ defined by

$$
\begin{aligned}
\mathcal{E}\left(x^{1}, x^{2}, x^{3}\right)= & \frac{1-\xi}{\gamma} H\left(x^{1}, x^{1}\right)+(2-\gamma-\xi) H\left(x^{2}, x^{2}\right)+\frac{1-\gamma}{\xi} H\left(x^{3}, x^{3}\right) \\
& +\frac{1-\xi}{\gamma} G\left(x^{1}, x^{2}\right)+\frac{(1-\xi)(1-\gamma)}{\gamma \xi} G\left(x^{1}, x^{3}\right)+\frac{1-\gamma}{\xi} G\left(x^{3}, x^{2}\right) .
\end{aligned}
$$

\section{Proof of Theorem 1.5}

\subsection{Construction of the approximate solution}

We denote by $\varepsilon$ the smallest positive parameter satisfying

$$
\rho^{4}=\frac{384 \varepsilon^{4}}{\left(1+\varepsilon^{2}\right)^{4}}
$$

Let

$$
u_{\varepsilon}(x):=4 \ln \left(1+\varepsilon^{2}\right)-4 \ln \left(\varepsilon^{2}+|x|^{2}\right),
$$

which is a solution of

$$
\Delta^{2} u=\rho^{4} e^{u} \quad \text { in } \mathbb{R}^{4} .
$$


Hence for all $\tau>0$ the function

$$
u_{\varepsilon, \tau}(x):=4 \ln \left(1+\varepsilon^{2}\right)+4 \ln \tau-4 \ln \left(\varepsilon^{2}+|\tau x|^{2}\right)
$$

is also a solution to 3.1 .

\subsubsection{A linearized operator}

First we introduce some definitions and notations.

Definition 3.1. Given $k \in \mathbb{N}, \alpha \in(0,1), \mu \in \mathbb{R}$ and $|x|=r$, we define the Hölder weighted space $\mathcal{C}_{\mu}^{k, \alpha}\left(\mathbb{R}^{4}\right)$ as the space of functions $w \in \mathcal{C}_{\text {loc }}^{k, \alpha}\left(\mathbb{R}^{4}\right)$ for which the following norm

$$
\|u\|_{\mathcal{C}_{\mu}^{k, \alpha}\left(\mathbb{R}^{4}\right)}=\|u\|_{\mathcal{C}^{k, \alpha}\left(\bar{B}_{1}(0)\right)}+\sup _{r \geq 1}\left(\left(1+r^{2}\right)^{-\mu / 2}\|u(r \cdot)\|_{\mathcal{C}^{k, \alpha}\left(\bar{B}_{1}(0)-B_{1 / 2}(0)\right)}\right)
$$

is finite. Similarly, for given $\bar{r} \geq 1$, let $\mathcal{C}_{\mu}^{k, \alpha}\left(B_{\bar{r}}(0)\right)$ be the space of functions in $\mathcal{C}^{k, \alpha}\left(B_{\bar{r}}(0)\right)$ for which the following norm

$$
\|u\|_{\mathcal{C}_{\mu}^{k, \alpha}\left(B_{\bar{r}}(0)\right)}=\|u\|_{\mathcal{C}^{k, \alpha}\left(B_{1}(0)\right)}+\sup _{1 \leq r \leq \bar{r}}\left(r^{-\mu}\|u(r \cdot)\|_{\mathcal{C}^{k, \alpha}\left(\bar{B}_{1}(0)-B_{1 / 2}(0)\right)}\right)
$$

is finite. Finally, set $B_{r}^{*}\left(x^{i}\right)=B_{r}\left(x^{i}\right)-\left\{x^{i}\right\}$, let $\mathcal{C}_{\mu}^{k, \alpha}\left(\bar{B}_{1}^{*}(0)\right)$ be the space of functions in $\mathcal{C}_{\text {loc }}^{k, \alpha}\left(\bar{B}_{1}^{*}(0)\right)$ for which the following norm

$$
\|u\|_{\mathcal{C}_{\mu}^{k, \alpha}\left(\bar{B}_{1}^{*}(0)\right)}=\sup _{r \leq 1 / 2}\left(r^{-\mu}\|u(r \cdot)\|_{\mathcal{C}^{k, \alpha}\left(\bar{B}_{2}(0)-B_{1}(0)\right)}\right)
$$

is finite.

We define the linear elliptic operator $\mathbb{L}$ by

$$
\mathbb{L}:=\Delta^{2}-\frac{384}{\left(1+r^{2}\right)^{4}},
$$

which is the linearized operator of $\Delta^{2} u-\rho^{4} e^{u}=0$ about the radial symmetric solution $u_{\varepsilon=1, \tau=1}$ defined by 3.2 . When $k \geq 2$, we let $\left[\mathcal{C}_{\mu}^{k, \alpha}(\bar{\Omega})\right]_{0}$ to be the subspace of functions $w \in \mathcal{C}_{\mu}^{k, \alpha}(\bar{\Omega})$ satisfying $\Delta w=w=0$ on $\partial \Omega$.

For all $\varepsilon, \tau_{i}>0, i=1,2,3$ and $\gamma, \xi \in(0,1)$, we define

$$
r_{\varepsilon}:=\max \left(\varepsilon^{1 / 2}, \varepsilon^{(\gamma+\xi-1) / \gamma}, \varepsilon^{(\gamma+\xi-1) / \xi}\right) \quad \text { and } \quad R_{\varepsilon}^{i}:=\tau_{i} \frac{r_{\varepsilon}}{\varepsilon} .
$$

Proposition 3.2. 4 All bounded solutions of $\mathbb{L} w=0$ on $\mathbb{R}^{4}$ are linear combination of

$$
\phi_{0}(x)=4 \frac{1-|x|^{2}}{1+|x|^{2}} \quad \text { and } \quad \phi_{i}(x)=\frac{8 x_{i}}{1+|x|^{2}} \quad \text { for } i=1, \ldots, 4 \text {. }
$$

Moreover, for $\mu>1, \mu \notin \mathbb{Z}$, the operator $\mathbb{L}: \mathcal{C}_{\mu}^{4, \alpha}\left(\mathbb{R}^{4}\right) \rightarrow \mathcal{C}_{\mu-4}^{0, \alpha}\left(\mathbb{R}^{4}\right)$ is surjective. 
In the following, we denote by $\mathcal{G}_{\mu}$ to be a right inverse of $\mathbb{L}$. Similarly, using the fact that any bounded bi-harmonic solution on $\mathbb{R}^{4}$ is constant, we claim

Proposition 3.3. Let $\delta>0, \delta \notin \mathbb{Z}$ then $\Delta^{2}$ is surjective from $\mathcal{C}_{\delta}^{4, \alpha}\left(\mathbb{R}^{4}\right)$ to $\mathcal{C}_{\delta-4}^{0, \alpha}\left(\mathbb{R}^{4}\right)$.

We denote by $\mathcal{K}_{\delta}: \mathcal{C}_{\delta-4}^{0, \alpha}\left(\mathbb{R}^{4}\right) \rightarrow \mathcal{C}_{\delta}^{4, \alpha}\left(\mathbb{R}^{4}\right)$ a right inverse of $\Delta^{2}$ for $\delta>0, \delta \notin \mathbb{Z}$.

Finally, we consider punctured domains. Given $\widetilde{x}^{1}, \widetilde{x}^{2}, \widetilde{x}^{3}$ three distinct points in $\Omega$, we define $\widetilde{\boldsymbol{x}}:=\left(\widetilde{x}^{1}, \widetilde{x}^{2}, \widetilde{x}^{3}\right)$ and $\bar{\Omega}^{*}(\widetilde{\boldsymbol{x}}):=\bar{\Omega}-\left\{\widetilde{x}^{1}, \widetilde{x}^{2}, \widetilde{x}^{3}\right\}$. Let $r_{0}>0$ be small such that $\bar{B}_{r_{0}}\left(\widetilde{x}^{i}\right)$ are disjoint and included in $\Omega$. For all $r \in\left(0, r_{0}\right)$, we define

$$
\bar{\Omega}_{r}(\widetilde{\boldsymbol{x}}):=\bar{\Omega}-\bigcup_{i=1}^{3} B_{r}\left(\widetilde{x}^{i}\right)
$$

Definition 3.4. Let $k \in \mathbb{R}, \alpha \in(0,1)$ and $\nu \in \mathbb{R}$, we introduce the Hölder weighted space $\mathcal{C}_{\nu}^{k, \alpha}\left(\bar{\Omega}^{*}(\widetilde{\boldsymbol{x}})\right)$ as the space of functions $w \in \mathcal{C}_{\text {loc }}^{k, \alpha}\left(\bar{\Omega}^{*}(\widetilde{\boldsymbol{x}})\right)$ such that

$$
\|w\|_{\mathcal{C}_{\nu}^{k, \alpha}\left(\bar{\Omega}^{*}(\widetilde{\boldsymbol{x}})\right)}:=\|w\|_{\mathcal{C}^{k, \alpha}\left(\bar{\Omega}_{r_{0} / 2}(\widetilde{\boldsymbol{x}})\right)}+\sum_{i=1}^{3} \sup _{0<r \leq r_{0} / 2}\left(r^{-\nu}\left\|w\left(\widetilde{x}^{i}+r \cdot\right)\right\|_{\mathcal{C}^{k, \alpha}\left(\bar{B}_{2}(0)-B_{1}(0)\right)}\right)
$$

is finite. Furthermore, for $k \geq 2$, let $\left[\mathcal{C}_{\nu}^{k, \alpha}\left(\bar{\Omega}^{*}(\widetilde{\boldsymbol{x}})\right)\right]_{0}$ to be the set of $w \in \mathcal{C}_{\nu}^{k, \alpha}\left(\bar{\Omega}^{*}(\widetilde{\boldsymbol{x}})\right)$ satisfying $\Delta w=w=0$ on $\partial \Omega$.

We recall the following result.

Proposition 3.5. 12 Let $\nu<0, \nu \notin \mathbb{Z}$ then $\Delta^{2}$ is surjective from $\left[\mathcal{C}_{\nu}^{4, \alpha}\left(\bar{\Omega}^{*}(\widetilde{\boldsymbol{x}})\right)\right]_{0}$ to $\mathcal{C}_{\nu-4}^{0, \alpha}\left(\bar{\Omega}^{*}(\widetilde{\boldsymbol{x}})\right)$

We denote by $\widetilde{\mathcal{G}}_{\nu}: \mathcal{C}_{\nu-4}^{0, \alpha}\left(\bar{\Omega}^{*}(\widetilde{\boldsymbol{x}})\right) \rightarrow\left[\mathcal{C}_{\nu}^{4, \alpha}\left(\bar{\Omega}^{*}(\widetilde{\boldsymbol{x}})\right)\right]_{0}$ a right inverse of $\Delta^{2}$ for $\nu<0$, $\nu \notin \mathbb{Z}$.

\subsubsection{Ansatz and first estimates}

For all $\sigma \geq 1$, we denote by $\xi_{\mu, \sigma}: \mathcal{C}_{\mu}^{0, \alpha}\left(\bar{B}_{\sigma}(0)\right) \rightarrow \mathcal{C}_{\mu}^{0, \alpha}\left(\mathbb{R}^{4}\right)$ the extension operator defined by

$$
\begin{cases}\xi_{\mu, \sigma}(f)(x) \equiv f(x) & \text { for }|x| \leq \sigma \\ \xi_{\mu, \sigma}(f)(x)=\chi\left(\frac{|x|}{\sigma}\right) f\left(\sigma \frac{x}{|x|}\right) & \text { for }|x| \geq \sigma\end{cases}
$$

Here $\chi$ is a cut-off function over $\mathbb{R}_{+}$, which is equal to 1 for $t \leq 1$ and equal to 0 for $t \geq 2$.

It is easy to check that there exists a constant $c=\bar{c}(\mu)>0$, independent of $\sigma$ such that

$$
\left\|\xi_{\mu, \sigma}(w)\right\|_{\mathcal{C}_{\mu}^{0, \alpha}\left(\mathbb{R}^{4}\right)} \leq \bar{c}\|w\|_{\mathcal{C}_{\mu}^{0, \alpha}\left(\bar{B}_{\sigma}(0)\right)}
$$


Now we define an ansatz for solution of (1.1):

$$
\widetilde{u}_{1}(x)= \begin{cases}\frac{1}{\gamma} u_{\varepsilon, \tau_{1}}\left(x-x^{1}\right)-\frac{1-\gamma}{\gamma} G\left(x, x^{2}\right)-\frac{1-\gamma}{\gamma \xi} G\left(x, x^{3}\right)-\frac{\ln \gamma}{\gamma}, & x \in B_{r_{\varepsilon}}\left(x^{1}\right), \\ u_{\varepsilon, \tau_{2}}\left(x-x^{2}\right), & x \in B_{r_{\varepsilon}}\left(x^{2}\right), \\ \frac{1}{\gamma} G\left(x, x^{1}\right)+G\left(x, x^{2}\right), & x \in \Omega \backslash \bigcup_{i=1}^{2} B_{r_{\varepsilon}}\left(x^{i}\right)\end{cases}
$$

and

$$
\widetilde{u}_{2}(x)= \begin{cases}\frac{1}{\xi} u_{\varepsilon, \tau_{3}}\left(x-x^{3}\right)-\frac{1-\xi}{\xi} G\left(x, x^{2}\right)-\frac{1-\xi}{\gamma \xi} G\left(x, x^{1}\right)-\frac{\ln \xi}{\xi}, & x \in B_{r_{\varepsilon}}\left(x^{3}\right), \\ u_{\varepsilon, \tau_{2}}\left(x-x^{2}\right), & x \in B_{r_{\varepsilon}}\left(x^{2}\right), \\ \frac{1}{\xi} G\left(x, x^{3}\right)+G\left(x, x^{2}\right), & x \in \Omega \backslash \bigcup_{i=2}^{3} B_{r_{\varepsilon}}\left(x^{i}\right) .\end{cases}
$$

Therefore, in $B_{r_{\varepsilon}}\left(x^{1}\right)$, there holds

$$
\begin{aligned}
& \Delta^{2} \widetilde{u}_{1}-\rho^{4} e^{\gamma \widetilde{u}_{1}+(1-\gamma) \widetilde{u}_{2}}=0 \\
& \Delta^{2} \widetilde{u}_{2}-\rho^{4} e^{\xi \widetilde{u}_{2}+(1-\xi) \widetilde{u}_{1}}=\frac{-384 \varepsilon^{4} \tau_{1}^{4 \frac{1-\xi}{\gamma}}\left(1+\varepsilon^{2}\right)^{4 \frac{1-\gamma-\xi}{\gamma}}}{\gamma^{\frac{1-\xi}{\gamma}}\left(\varepsilon^{2}+\tau_{1}^{2}\left|x-x^{1}\right|^{2}\right)^{4 \frac{1-\xi}{\gamma}}} e^{\frac{\gamma+\xi-1}{\gamma} G\left(x, x^{2}\right)+\frac{\gamma+\xi-1}{\gamma \xi} G\left(x, x^{3}\right)} .
\end{aligned}
$$

Then, for $r=\left|x-x^{1}\right|$ and $0<\delta<(\gamma+\xi-1) / \gamma$, we have

$$
\begin{aligned}
\left\|\Delta^{2} \widetilde{u}_{2}-\rho^{4} e^{\xi \widetilde{u}_{2}+(1-\xi) \widetilde{u}_{1}}\right\|_{\mathcal{C}_{\delta-4}^{0, \alpha}\left(B_{r_{\varepsilon}}\left(x^{1}\right)\right)} & \leq C \sup _{r<r_{\varepsilon}} \frac{\tau_{1}^{4 \frac{1-\xi}{\gamma}} \varepsilon^{4}}{\gamma^{\frac{1-\xi}{\gamma}}\left(1+\varepsilon^{2}\right)^{4-4 \frac{1-\xi}{\gamma}}} \frac{r^{4-\delta}}{\varepsilon^{8 \frac{1-\xi}{\gamma}}\left(1+\left(\frac{\tau_{1}}{\varepsilon} r\right)^{2}\right)^{4 \frac{1-\xi}{\gamma}}} \\
& \leq C \sup _{r<R_{\varepsilon}^{1}} \frac{\varepsilon^{8-8 \frac{1-\xi}{\gamma}-\delta}}{\left(1+\varepsilon^{2}\right)^{4-4 \frac{1-\xi}{\gamma}}} \frac{r^{4-\delta}}{\left(1+r^{2}\right)^{4 \frac{1-\xi}{\gamma}}} \\
& \leq C \sup _{r<R_{\varepsilon}^{1}} \varepsilon^{8-8 \frac{1-\xi}{\gamma}-\delta} S(r),
\end{aligned}
$$

where $S(r)=\frac{r^{4-\delta}}{\left(1+r^{2}\right)^{4(1-\xi) / \gamma}}$.

If $4-\delta-8(1-\xi) / \gamma \leq 0$, then $S$ is bounded on $\mathbb{R}_{+}$, hence

$$
\left\|\Delta^{2} \widetilde{u}_{2}-\rho^{4} e^{\xi \widetilde{u}_{2}+(1-\xi) \widetilde{u}_{1}}\right\|_{\mathcal{C}_{\delta-4}^{0, \alpha}\left(B_{r_{\varepsilon}}\left(x^{1}\right)\right)} \leq C \varepsilon^{8-8 \frac{1-\xi}{\gamma}-\delta} \leq C r_{\varepsilon}^{2}
$$

If $4-\delta-8(1-\xi) / \gamma>0, \sup _{\left[0, r_{\varepsilon} / \varepsilon\right)} S(r)=S\left(\frac{r_{\varepsilon}}{\varepsilon}\right)$, then

$$
\left\|\Delta^{2} \widetilde{u}_{2}-\rho^{4} e^{\xi \widetilde{u}_{2}+(1-\xi) \widetilde{u}_{1}}\right\|_{\mathcal{C}_{\delta-4}^{0, \alpha}\left(B_{r_{\varepsilon}}\left(x^{1}\right)\right)} \leq C r_{\varepsilon}^{2} \quad \text { as } \varepsilon^{8-8 \frac{1-\xi}{\gamma}-\delta} S\left(\frac{r_{\varepsilon}}{\varepsilon}\right) \leq C r_{\varepsilon}^{2} .
$$

Similarly in $B_{r_{\varepsilon}}\left(x^{3}\right)$, there holds

$$
\begin{aligned}
& \Delta^{2} \widetilde{u}_{1}-\rho^{4} e^{\gamma \widetilde{u}_{1}+(1-\gamma) \widetilde{u}_{2}}=\frac{-384 \varepsilon^{4} \tau_{3}^{4 \frac{1-\gamma}{\xi}}\left(1+\varepsilon^{2}\right)^{4 \frac{1-\gamma-\xi}{\xi}}}{\xi^{\frac{1-\gamma}{\xi}}\left(\varepsilon^{2}+\tau_{3}^{2}\left|x-x^{3}\right|^{2}\right)^{4 \frac{1-\gamma}{\xi}}} e^{\frac{\gamma+\xi-1}{\xi} G\left(x, x^{2}\right)+\frac{\gamma+\xi-1}{\gamma \xi} G\left(x, x^{1}\right)}, \\
& \Delta^{2} \widetilde{u}_{2}-\rho^{4} e^{\xi \widetilde{u}_{2}+(1-\xi) \widetilde{u}_{1}}=0 .
\end{aligned}
$$


Then, for $r=\left|x-x^{3}\right|$ and $0<\delta<(\gamma+\xi-1) / \xi$, we have the same estimates

$$
\left\|\Delta^{2} \widetilde{u}_{1}-\rho^{4} e^{\gamma \widetilde{u}_{1}+(1-\gamma) \widetilde{u}_{2}}\right\|_{\mathcal{C}_{\delta-4}^{0, \alpha}\left(B_{r_{\varepsilon}}\left(x^{3}\right)\right)} \leq C r_{\varepsilon}^{2}
$$

Finally, in $B_{r_{\varepsilon}}\left(x^{2}\right)$, we have an exact solution of the system.

\subsubsection{Bi-harmonic extensions}

Next, we will study the properties of interior and exterior bi-harmonic extensions.

Given $(\varphi, \psi),(\widetilde{\varphi}, \widetilde{\psi}) \in \mathcal{C}^{4, \alpha}\left(S^{3}\right) \times \mathcal{C}^{2, \alpha}\left(S^{3}\right)$, we define respectively $H^{\text {int }}=H^{\text {int }}(\varphi, \psi ; \cdot)=$ $H_{\varphi, \psi}^{\text {int }}$ and $H^{\text {ext }}=H^{\operatorname{ext}}(\widetilde{\varphi}, \widetilde{\psi} ; \cdot)=H_{\widetilde{\varphi}, \widetilde{\psi}}^{\text {ext }}$ to be the solution of

$$
\left\{\begin{array} { l l } 
{ \Delta ^ { 2 } H ^ { \text { int } } = 0 } & { \text { in } B _ { 1 } ( 0 ) , } \\
{ H ^ { \text { int } } = \varphi } & { \text { on } \partial B _ { 1 } ( 0 ) , } \\
{ \Delta H ^ { \text { int } } = \psi } & { \text { on } \partial B _ { 1 } ( 0 ) , }
\end{array} \text { and } \quad \left\{\begin{array}{ll}
\Delta^{2} H^{\text {ext }}=0 & \text { in } \mathbb{R}^{4}-B_{1}(0), \\
H^{\text {ext }}=\widetilde{\varphi} & \text { on } \partial B_{1}(0) \\
\Delta H^{\text {ext }}=\widetilde{\psi} & \text { on } \partial B_{1}(0)
\end{array}\right.\right.
$$

which decays at infinity. We will also use

Definition 3.6. Given $k \in \mathbb{N}, \alpha \in(0,1)$ and $\nu \in \mathbb{R}$, we define the space $\mathcal{C}_{\nu}^{k, \alpha}\left(\mathbb{R}^{4}-B_{1}(0)\right)$ as the space of functions $w \in \mathcal{C}_{\text {loc }}^{k, \alpha}\left(\mathbb{R}^{4}-B_{1}(0)\right)$ for which the following norm

$$
\|w\|_{\mathcal{C}_{\nu}^{k, \alpha}\left(\mathbb{R}^{4}-B_{1}(0)\right)}=\sup _{r \geq 1}\left(r^{-\nu}\|w(r \cdot)\|_{\mathcal{C}_{\nu}^{k, \alpha}\left(\bar{B}_{2}(0)-B_{1}(0)\right)}\right)
$$

is finite.

We denote by $e_{1}, \ldots, e_{4}$ the coordinate functions on $S^{3}$.

Lemma 3.7. 2] Assume that

$$
\int_{S^{3}}(8 \varphi-\psi) d v_{S^{3}}=0 \quad \text { and } \quad \int_{S^{3}}(12 \varphi-\psi) e_{\ell} d v_{S^{3}}=0 \quad \text { for } \ell=1, \ldots, 4 .
$$

Then there exists $c>0$ such that

$$
\left\|H_{\varphi, \psi}^{\mathrm{int}}\right\|_{\mathcal{C}_{2}^{4, \alpha}\left(\bar{B}_{1}^{*}(0)\right)} \leq c\left(\|\varphi\|_{\mathcal{C}^{4, \alpha}\left(S^{3}\right)}+\|\psi\|_{\mathcal{C}^{2, \alpha}\left(S^{3}\right)}\right) .
$$

Similarly, there exists $c>0$ such that if

$$
\int_{S^{3}} \widetilde{\psi} d v_{S^{3}}=0
$$

then

$$
\left\|H_{\widetilde{\varphi}, \widetilde{\psi}}^{\mathrm{ext}}\right\|_{\mathcal{C}_{-1}^{4, \alpha}\left(\mathbb{R}^{4}-B_{1}(0)\right)} \leq c\left(\|\widetilde{\varphi}\|_{\mathcal{C}^{4, \alpha}\left(S^{3}\right)}+\|\widetilde{\psi}\|_{\mathcal{C}^{2, \alpha}\left(S^{3}\right)}\right) .
$$

If $F \subset L^{2}\left(S^{3}\right)$ be a subspace $S^{3}$, we denote $F^{\perp}$ to be the subspace of $F$ which are $L^{2}\left(S^{3}\right)$-orthogonal to the functions $1, e_{1}, \ldots, e_{4}$. We will need the following result. 
Lemma 3.8. 2] The mapping

$$
\begin{aligned}
& \mathcal{P}: \quad \mathcal{C}^{4, \alpha}\left(S^{3}\right)^{\perp} \times \mathcal{C}^{2, \alpha}\left(S^{3}\right)^{\perp} \quad \longrightarrow \quad \mathcal{C}^{3, \alpha}\left(S^{3}\right)^{\perp} \times \mathcal{C}^{1, \alpha}\left(S^{3}\right)^{\perp} \\
& (\varphi, \psi) \quad \longmapsto\left(\partial_{r}\left(H_{\varphi, \psi}^{\mathrm{int}}-H_{\varphi, \psi}^{\mathrm{ext}}\right), \partial_{r}\left(\Delta H_{\varphi, \psi}^{\mathrm{int}}-\Delta H_{\varphi, \psi}^{\mathrm{ext}}\right)\right)
\end{aligned}
$$

is an isomorphism.

3.2. The nonlinear interior problem

Here, we are interested to study the system

$$
\Delta^{2} u_{1}=\rho^{4} e^{\gamma u_{1}+(1-\gamma) u_{2}}, \quad \Delta^{2} u_{2}=\rho^{4} e^{\xi u_{2}+(1-\xi) u_{1}} .
$$

Using the following transformations

$$
\begin{aligned}
& \begin{cases}v_{1}(x)=u_{1}\left(\frac{\varepsilon}{\tau_{1}} x\right)+\frac{8}{\gamma} \ln \varepsilon-\frac{4}{\gamma} \ln \left(\frac{\tau_{1}\left(1+\varepsilon^{2}\right)}{2}\right) & \text { in } B_{r_{\varepsilon}}\left(x^{1}\right), \\
v_{2}(x)=u_{2}\left(\frac{\varepsilon}{\tau_{1}} x\right) & \text { in } B_{r_{\varepsilon}}\left(x^{1}\right),\end{cases} \\
& \begin{cases}v_{1}(x)=u_{1}\left(\frac{\varepsilon}{\tau_{2}} x\right)+8 \ln \varepsilon-4 \ln \left(\frac{\tau_{2}\left(1+\varepsilon^{2}\right)}{2}\right) & \text { in } B_{r_{\varepsilon}}\left(x^{2}\right), \\
v_{2}(x)=u_{2}\left(\frac{\varepsilon}{\tau_{2}} x\right)+8 \ln \varepsilon-4 \ln \left(\frac{\tau_{2}\left(1+\varepsilon^{2}\right)}{2}\right) & \text { in } B_{r_{\varepsilon}}\left(x^{2}\right)\end{cases}
\end{aligned}
$$

and

$$
\begin{cases}v_{1}(x)=u_{1}\left(\frac{\varepsilon}{\tau_{3}} x\right) & \text { in } B_{r_{\varepsilon}}\left(x^{3}\right), \\ v_{2}(x)=u_{2}\left(\frac{\varepsilon}{\tau_{3}} x\right)+\frac{8}{\xi} \ln \varepsilon-\frac{4}{\xi} \ln \left(\frac{\tau_{3}\left(1+\varepsilon^{2}\right)}{2}\right) & \text { in } B_{r_{\varepsilon}}\left(x^{3}\right) .\end{cases}
$$

So the previous systems can be written as

$$
\begin{gathered}
\begin{cases}\Delta^{2} v_{1}=24 e^{\gamma v_{1}+(1-\gamma) v_{2}} & \text { in } B_{R_{\varepsilon}^{1}}\left(x^{1}\right), \\
\Delta^{2} v_{2}=24 C_{1, \varepsilon}^{4 \frac{\gamma+\xi-1}{\gamma}} \varepsilon^{8 \frac{\gamma+\xi-1}{\gamma}} e^{\xi v_{2}+(1-\xi) v_{1}} & \text { in } B_{R_{\varepsilon}^{1}}\left(x^{1}\right),\end{cases} \\
\begin{cases}\Delta^{2} v_{1}=24 e^{\gamma v_{1}+(1-\gamma) v_{2}} & \text { in } B_{R_{\varepsilon}^{2}}\left(x^{2}\right), \\
\Delta^{2} v_{2}=24 e^{\xi v_{2}+(1-\xi) v_{1}} & \text { in } B_{R_{\varepsilon}^{2}}\left(x^{2}\right)\end{cases}
\end{gathered}
$$

and

$$
\begin{cases}\Delta^{2} v_{1}=24 C_{3, \varepsilon}^{4 \frac{\gamma+\xi-1}{\xi}} \varepsilon^{8 \frac{\gamma+\xi-1}{\xi}} e^{\gamma v_{1}+(1-\gamma) v_{2}} & \text { in } B_{R_{\varepsilon}^{3}}\left(x^{3}\right), \\ \Delta^{2} v_{2}=24 e^{\xi v_{2}+(1-\xi) v_{1}} & \text { in } B_{R_{\varepsilon}^{3}}\left(x^{3}\right),\end{cases}
$$

where $C_{i, \varepsilon}=\frac{2}{\tau_{i}\left(1+\varepsilon^{2}\right)}$ for $i=1,3$. Here $\tau_{i}>0$ is a constant which will be fixed later.

Given $\varphi^{i}:=\left(\varphi_{1}^{i}, \varphi_{2}^{i}\right) \in\left(\mathcal{C}^{4, \alpha}\left(S^{3}\right)\right)^{2}$ and $\psi^{i}:=\left(\psi_{1}^{i}, \psi_{2}^{i}\right) \in\left(\mathcal{C}^{2, \alpha}\left(S^{3}\right)\right)^{2}$ such that $\left(\varphi_{1}^{i}, \psi_{1}^{i}\right)$ and $\left(\varphi_{2}^{i}, \psi_{2}^{i}\right)$ satisfy $(3.5)$. We denote by $\bar{u}=u_{\varepsilon=1, \tau_{i}=1}$, we write for $x \in B_{R_{\varepsilon}^{1}}\left(x^{1}\right)$ the 
following system

$$
\begin{aligned}
v_{1}(x)= & \frac{1}{\gamma} \bar{u}\left(x-x^{1}\right)-\frac{1-\gamma}{\gamma} G\left(\frac{\varepsilon x}{\tau_{1}}, x^{2}\right)-\frac{1-\gamma}{\gamma \xi} G\left(\frac{\varepsilon x}{\tau_{1}}, x^{3}\right) \\
& -\frac{\ln \gamma}{\gamma}+H^{\operatorname{int}}\left(\varphi_{1}^{1}, \psi_{1}^{1} ; \frac{x-x^{1}}{R_{\varepsilon}^{1}}\right)+h_{1}^{1}(x), \\
v_{2}(x)= & \frac{1}{\xi} G\left(\frac{\varepsilon x}{\tau_{1}}, x^{3}\right)+G\left(\frac{\varepsilon x}{\tau_{1}}, x^{2}\right)+H^{\text {int }}\left(\varphi_{2}^{1}, \psi_{2}^{1} ; \frac{x-x^{1}}{R_{\varepsilon}^{1}}\right)+h_{2}^{1}(x) .
\end{aligned}
$$

Using the fact that $H^{\text {int }}$ is bi-harmonic and that $e^{\bar{u}\left(x-x^{1}\right)}=\frac{16}{\left(1+\left|x-x^{1}\right|^{2}\right)^{4}}$, we see that this amounts to solve the system

$$
\begin{aligned}
\mathbb{L} h_{1}^{1}= & \frac{384}{\gamma\left(1+r^{2}\right)^{4}}\left(e^{\gamma\left(h_{1}^{1}+H_{\varphi_{1}^{1}, \psi_{1}^{1}}^{\mathrm{int}}\right)+(1-\gamma)\left(h_{2}^{1}+H_{\varphi_{2}^{1}, \psi_{2}^{1}}^{\mathrm{int}}\right)}-\gamma h_{1}^{1}-1\right), \\
\Delta^{2} h_{2}^{1}= & \frac{24 C_{1, \varepsilon}^{4 \frac{\gamma+\xi-1}{\gamma}} 16^{\frac{1-\xi}{\gamma}} \varepsilon^{8 \frac{\gamma+\xi-1}{\gamma}}}{\gamma^{\frac{1-\xi}{\gamma}}\left(1+r^{2}\right)^{4 \frac{1-\xi}{\gamma}}} \\
& \times e^{\frac{\gamma+\xi-1}{\gamma} G\left(\frac{\varepsilon x}{\tau_{1}}, x^{2}\right)+\frac{\gamma+\xi-1}{\gamma \xi} G\left(\frac{\varepsilon x}{\tau_{1}}, x^{3}\right)+\xi\left(h_{2}^{1}+H_{\varphi_{2}^{1}, \psi_{2}^{1}}^{\mathrm{int}}\right)+(1-\xi)\left(h_{1}^{1}+H_{\varphi_{1}^{1}, \psi_{1}^{1}}^{\mathrm{int}}\right)} .
\end{aligned}
$$

We denote by

$$
\mathbb{L} h_{1}^{1}=\mathcal{T}_{1}\left(h_{1}^{1}, h_{2}^{1}\right) \quad \text { and } \quad \Delta^{2} h_{2}^{1}=\mathcal{T}_{2}\left(h_{1}^{1}, h_{2}^{1}\right) .
$$

Fix $\mu \in(1,2)$ and $\delta \in\left(0, \min \left\{\left(\frac{\gamma+\xi-1}{\gamma}\right),\left(\frac{\gamma+\xi-1}{\xi}\right)\right\}\right)$. To find a solution of $(3.11)$, it is enough to find a fixed point $\left(h_{1}^{1}, h_{2}^{1}\right)$ in a small ball of $\mathcal{C}_{\mu}^{4, \alpha}\left(\mathbb{R}^{4}\right) \times \mathcal{C}_{\delta}^{4, \alpha}\left(\mathbb{R}^{4}\right)$ solutions of

$$
\begin{aligned}
& h_{1}^{1}=\mathcal{G}_{\mu} \circ \xi_{\mu, R_{\varepsilon}^{1}} \circ \mathcal{T}_{1}\left(h_{1}^{1}, h_{2}^{1}\right)=\mathcal{N}_{1}\left(h_{1}^{1}, h_{2}^{1}\right), \\
& h_{2}^{1}=\mathcal{K}_{\delta} \circ \xi_{\delta, R_{\varepsilon}^{1}} \circ \mathcal{T}_{2}\left(h_{1}^{1}, h_{2}^{1}\right)=\mathcal{M}_{1}\left(h_{1}^{1}, h_{2}^{1}\right) .
\end{aligned}
$$

Here $\xi_{\mu, R_{\varepsilon}^{1}}$ is defined in $(3.3), \mathcal{G}_{\mu}$ and $\mathcal{K}_{\delta}$ are defined after Propositions 3.2 and 3.3 , respectively.

Given $\kappa>0$ (whose value will be fixed later on), we further assume that the functions $\varphi_{j}^{1}$ and $\psi_{j}^{1}$ satisfy

$$
\left\|\varphi_{j}^{1}\right\|_{\mathcal{C}^{4, \alpha}\left(S^{3}\right)} \leq \kappa r_{\varepsilon}^{2} \quad \text { and } \quad\left\|\psi_{j}^{1}\right\|_{\mathcal{C}^{2, \alpha}\left(S^{3}\right)} \leq \kappa r_{\varepsilon}^{2} \quad \text { for } j=1,2 .
$$

Then we have the following result.

Lemma 3.9. Let $\varphi^{1}:=\left(\varphi_{1}^{1}, \varphi_{2}^{1}\right) \in\left(\mathcal{C}^{4, \alpha}\left(S^{3}\right)\right)^{2}$ and $\psi^{1}:=\left(\psi_{1}^{1}, \psi_{2}^{1}\right) \in\left(\mathcal{C}^{2, \alpha}\left(S^{3}\right)\right)^{2}$ such that $\left(\varphi_{1}^{1}, \psi_{1}^{1}\right)$ and $\left(\varphi_{2}^{1}, \psi_{2}^{1}\right)$ satisfy (3.5) and (3.13). Given $\kappa>0$, there exist $\varepsilon_{\kappa}>0$, $c_{\kappa}>0$ and $\gamma_{0} \in(0,1)$ such that for all $\varepsilon \in\left(0, \varepsilon_{\kappa}\right), \gamma \in\left(\gamma_{0}, 1\right), \mu \in(1,2)$ and $\delta \in$ $\left(0, \min \left\{\left(\frac{\gamma+\xi-1}{\gamma}\right),\left(\frac{\gamma+\xi-1}{\xi}\right)\right\}\right)$. We have

$$
\begin{gathered}
\left\|\mathcal{N}_{1}(0,0)\right\|_{\mathcal{C}_{\mu}^{4, \alpha}\left(\mathbb{R}^{4}\right)} \leq c_{\kappa} r_{\varepsilon}^{2}, \quad\left\|\mathcal{M}_{1}(0,0)\right\|_{\mathcal{C}_{\delta}^{4, \alpha}\left(\mathbb{R}^{4}\right)} \leq c_{\kappa} r_{\varepsilon}^{2}, \\
\left\|\mathcal{N}_{1}\left(h_{1}^{1}, h_{2}^{1}\right)-\mathcal{N}_{1}\left(k_{1}^{1}, k_{2}^{1}\right)\right\|_{\mathcal{C}_{\mu}^{4, \alpha}\left(\mathbb{R}^{4}\right)} \leq c_{\kappa} r_{\varepsilon}^{2}\left\|h_{1}^{1}-k_{1}^{1}\right\|_{\mathcal{C}_{\mu}^{4, \alpha}\left(\mathbb{R}^{4}\right)}+c_{\kappa}(1-\gamma)\left\|h_{2}^{1}-k_{2}^{1}\right\|_{\mathcal{C}_{\delta}^{4, \alpha}\left(\mathbb{R}^{4}\right)}
\end{gathered}
$$


and

$$
\left\|\mathcal{M}_{1}\left(h_{1}^{1}, h_{2}^{1}\right)-\mathcal{M}_{1}\left(k_{1}^{1}, k_{2}^{1}\right)\right\|_{\mathcal{C}_{\delta}^{4, \alpha}\left(\mathbb{R}^{4}\right)} \leq c_{\kappa} r_{\varepsilon}^{2}\left\|\left(h_{1}^{1}, h_{2}^{1}\right)-\left(k_{1}^{1}, k_{2}^{1}\right)\right\|_{\mathcal{C}_{\mu}^{4, \alpha}\left(\mathbb{R}^{4}\right) \times \mathcal{C}_{\delta}^{4, \alpha}\left(\mathbb{R}^{4}\right)},
$$

provided $\left(h_{1}^{1}, h_{2}^{1}\right),\left(k_{1}^{1}, k_{2}^{1}\right) \in \mathcal{C}_{\mu}^{4, \alpha}\left(\mathbb{R}^{4}\right) \times \mathcal{C}_{\delta}^{4, \alpha}\left(\mathbb{R}^{4}\right)$ satisfying

$$
\left\|\left(h_{1}^{1}, h_{2}^{1}\right)\right\|_{\mathcal{C}_{\mu}^{4, \alpha}\left(\mathbb{R}^{4}\right) \times \mathcal{C}_{\delta}^{4, \alpha}\left(\mathbb{R}^{4}\right)} \leq 2 c_{\kappa} r_{\varepsilon}^{2}, \quad\left\|\left(k_{1}^{1}, k_{2}^{1}\right)\right\|_{\mathcal{C}_{\mu}^{4, \alpha}\left(\mathbb{R}^{4}\right) \times \mathcal{C}_{\delta}^{4, \alpha}\left(\mathbb{R}^{4}\right)} \leq 2 c_{\kappa} r_{\varepsilon}^{2}
$$

Proof. The proof of the first and the second estimates follows from the asymptotic behavior of $H^{\text {int }}$ together with the assumption on the norms of $\varphi_{j}^{1}$ and $\psi_{j}^{1}$ given by 3.13 and it follows from the estimate of $H^{\text {int }}$, given by Lemma 3.7, that

$$
\left\|H_{\varphi_{j}^{1}, \psi_{j}^{1}}^{\mathrm{int}}\left(\frac{r}{R_{\varepsilon}^{1}} \cdot\right)\right\|_{\mathcal{C}^{4, \alpha}\left(\bar{B}_{2}(0)-B_{1}(0)\right)} \leq C r^{2}\left(R_{\varepsilon}^{1}\right)^{-2}\left(\left\|\varphi_{j}^{1}\right\|_{\mathcal{C}^{4, \alpha}\left(S^{3}\right)}+\left\|\psi_{j}^{1}\right\|_{\mathcal{C}^{2, \alpha}\left(S^{3}\right)}\right)
$$

for all $r \leq R_{\varepsilon}^{1} / 2$. Then by 3.13 , we get

$$
\left\|H_{\varphi_{j}^{1}, \psi_{j}^{1}}^{\mathrm{int}}\left(\frac{r}{R_{\varepsilon}^{1}}\right)\right\|_{\mathcal{C}^{4, \alpha}\left(\bar{B}_{2}(0)-B_{1}(0)\right)} \leq c_{\kappa} \varepsilon^{2} r^{2} .
$$

On the other hand,

$$
\begin{aligned}
\sup _{r \leq R_{\varepsilon}^{1}} r^{4-\mu}\left|\mathcal{T}_{1}(0,0)\right| & \leq \sup _{r \leq R_{\varepsilon}^{1}} \frac{384 r^{4-\mu}}{\left(1+r^{2}\right)^{4}} \frac{1}{\gamma} \mid e^{\gamma H_{\varphi_{1}^{1}, \psi_{1}^{1}}^{\mathrm{int}}+(1-\gamma) H_{\varphi_{2}^{1}, \psi_{2}^{1}}^{\mathrm{int}}-1 \mid} \\
& \leq \sup _{r \leq R_{\varepsilon}^{1}} \frac{384 r^{4-\mu}}{\left(1+r^{2}\right)^{4}} \frac{1}{\gamma}\left(\gamma r^{2}\left\|H_{\varphi_{1}^{1}, \psi_{1}^{1}}^{\mathrm{int}}\right\|_{\mathcal{C}_{2}^{4, \alpha}}+(1-\gamma) r^{2}\left\|H_{\varphi_{2}^{1}, \psi_{2}^{1}}^{\mathrm{int}}\right\|_{\mathcal{C}_{2}^{4, \alpha}}\right) .
\end{aligned}
$$

Making use of Proposition 3.2 together with (3.4), for $\mu \in(1,2)$, we get that there exists $c_{\kappa}>0$ such that

$$
\left\|\mathcal{N}_{1}(0,0)\right\|_{\mathcal{C}_{\mu}^{4, \alpha}\left(\mathbb{R}^{4}\right)} \leq c_{\kappa} r_{\varepsilon}^{2}
$$

For the second estimate, we have

$$
\begin{aligned}
& \sup _{r \leq R_{\varepsilon}^{1}} r^{4-\delta}\left|\mathcal{T}_{2}(0,0)\right| \leq c \sup _{r \leq R_{\varepsilon}^{1}} C_{1, \varepsilon}^{4 \frac{\gamma+\xi-1}{\gamma}} \varepsilon^{8 \frac{\gamma+\xi-1}{\gamma}} r^{4-\delta}\left(\frac{16}{\left(1+r^{2}\right)^{4}}\right)^{\frac{1-\xi}{\gamma}} \\
& \times e^{\frac{\gamma+\xi-1}{\gamma} G\left(\frac{\varepsilon x}{\tau_{1}}, x^{2}\right)+\frac{\gamma+\xi-1}{\gamma \xi} G\left(\frac{\varepsilon x}{\tau_{1}}, x^{3}\right)+\xi H_{\varphi_{2}^{1}, \psi_{2}^{1}}^{\mathrm{int}}+(1-\xi) H_{\varphi_{1}^{1}, \psi_{1}^{1}}^{\mathrm{int}}} \\
& \leq c \sup _{r \leq R_{\varepsilon}^{1}} \varepsilon^{8 \frac{\gamma+\xi-1}{\gamma}} r^{4-\delta}\left(\frac{16}{\left(1+r^{2}\right)^{4}}\right)^{\frac{1-\xi}{\gamma}} \\
& \times\left(\xi r^{2}\left\|H_{\varphi_{2}^{1}, \psi_{2}^{1}}^{\mathrm{int}}\right\|_{\mathcal{C}_{2}^{4, \alpha}}+(1-\xi) r^{2}\left\|H_{\varphi_{1}^{1}, \psi_{1}^{1}}^{\mathrm{int}}\right\|_{\mathcal{C}_{2}^{4, \alpha}}+1\right) .
\end{aligned}
$$

Using the same argument as above, we get $\left\|\mathcal{M}_{1}(0,0)\right\|_{\mathcal{C}_{\delta}^{4, \alpha}\left(\mathbb{R}^{4}\right)} \leq c_{\kappa} r_{\varepsilon}^{2}$. 
To derive the third estimate, for $\left(h_{1}^{1}, h_{2}^{1}\right),\left(k_{1}^{1}, k_{2}^{1}\right)$ verifying (3.14), we have

$$
\begin{aligned}
& \sup _{r \leq R_{\varepsilon}^{1}} r^{4-\mu}\left|\mathcal{T}_{1}\left(h_{1}^{1}, h_{2}^{1}\right)-\mathcal{T}_{1}\left(k_{1}^{1}, k_{2}^{1}\right)\right| \\
\leq & \sup _{r \leq R_{\varepsilon}^{1}} \frac{384 r^{4-\mu}}{\left(1+r^{2}\right)^{4}} \frac{1}{\gamma} \mid\left(e^{\left.\gamma h_{1}^{1}+\gamma H_{\varphi_{1}^{1}, \psi_{1}^{1}}^{\mathrm{int}}+(1-\gamma) h_{2}^{1}+(1-\gamma) H_{\varphi_{2}^{1}, \psi_{2}^{1}}^{\mathrm{int}}-\gamma h_{1}^{1}-1\right)}\right. \\
& \quad-\left(e^{\left.\gamma k_{1}^{1}+\gamma H_{\varphi_{1}^{1}, \psi_{1}^{1}}^{\mathrm{int}}+(1-\gamma) k_{2}^{1}+(1-\gamma) H_{\varphi_{2}^{1}, \psi_{2}^{1}}^{\mathrm{int}}-\gamma k_{1}^{1}-1\right) \mid}\right. \\
\leq & c \sup _{r \leq R_{\varepsilon}^{1}} \frac{384 r^{4-\mu}}{\left(1+r^{2}\right)^{4}} \frac{1}{\gamma}\left[\gamma^{2}\left(\left(h_{1}^{1}\right)^{2}-\left(k_{1}^{1}\right)^{2}\right)+(1-\gamma)\left|h_{2}^{1}-k_{2}^{1}\right|\right] \\
\leq & c \sup _{r \leq R_{\varepsilon}^{1}} \frac{384 r^{4-\mu}}{\left(1+r^{2}\right)^{4}} \frac{1}{\gamma}\left[\gamma^{2} r^{2 \mu}\left(\left\|h_{1}^{1}\right\|_{\mathcal{C}_{\mu}^{4, \alpha}}+\left\|k_{1}^{1}\right\|_{\mathcal{C}_{\mu}^{4, \alpha}}\right)\left\|h_{1}^{1}-k_{1}^{1}\right\|_{\mathcal{C}_{\mu}^{4, \alpha}}+(1-\gamma) r^{\delta}\left\|h_{2}^{1}-k_{2}^{1}\right\|_{\mathcal{C}_{\delta}^{4, \alpha}}\right] .
\end{aligned}
$$

We conclude that

$$
\left\|\mathcal{N}_{1}\left(h_{1}^{1}, h_{2}^{1}\right)-\mathcal{N}_{1}\left(k_{1}^{1}, k_{2}^{1}\right)\right\|_{\mathcal{C}_{\mu}^{4, \alpha}\left(\mathbb{R}^{4}\right)} \leq c_{\kappa} r_{\varepsilon}^{2}\left\|h_{1}^{1}-k_{1}^{1}\right\|_{\mathcal{C}_{\mu}^{4, \alpha}\left(\mathbb{R}^{4}\right)}+c_{\kappa}(1-\gamma)\left\|h_{2}^{1}-k_{2}^{1}\right\|_{\mathcal{C}_{\delta}^{4, \alpha}\left(\mathbb{R}^{4}\right)} .
$$

On the other hand, we have

$$
\begin{aligned}
& \sup _{r \leq R_{\varepsilon}^{1}} r^{4-\delta}\left|\mathcal{T}_{2}\left(h_{1}^{1}, h_{2}^{1}\right)-\mathcal{T}_{2}\left(k_{1}^{1}, k_{2}^{1}\right)\right| \\
& \leq \sup _{r \leq R_{\varepsilon}^{1}} 24 C_{1, \varepsilon}^{4 \frac{\gamma+\xi-1}{\gamma}} \gamma^{-\frac{1-\xi}{\gamma}} \varepsilon^{8 \frac{\gamma+\xi-1}{\gamma}}\left(\frac{16}{\left(1+r^{2}\right)^{4}}\right)^{\frac{1-\xi}{\gamma}} r^{4-\delta} e^{\frac{\gamma+\xi-1}{\gamma} G\left(\frac{\varepsilon x}{\tau_{1}}, x^{2}\right)+\frac{\gamma+\xi-1}{\gamma \xi} G\left(\frac{\varepsilon x}{\tau_{1}}, x^{3}\right)} \\
& \times\left|e^{\xi h_{2}^{1}+\xi H_{\varphi_{2}^{1}, \psi_{2}^{1}}^{\mathrm{int}}+(1-\xi) h_{1}^{1}+(1-\xi) H_{\varphi_{1}^{1}, \psi_{1}^{1}}^{\mathrm{int}}}-e^{\xi k_{2}^{1}+\xi H_{\varphi_{2}^{1}, \psi_{2}^{1}}^{\mathrm{int}}+(1-\xi) k_{1}^{1}+(1-\xi) H_{\varphi_{1}^{1}, \psi_{1}^{1}}^{\mathrm{int}}}\right| \\
& \leq c \sup _{r \leq R_{\varepsilon}^{1}} 24 C_{1, \varepsilon}^{4 \frac{\gamma+\xi-1}{\gamma}} \gamma^{-\frac{1-\xi}{\gamma}} \varepsilon^{8 \frac{\gamma+\xi-1}{\gamma}}\left(\frac{16}{\left(1+r^{2}\right)^{4}}\right)^{\frac{1-\xi}{\gamma}} r^{4-\delta}\left[\xi\left|h_{2}^{1}-k_{2}^{1}\right|+(1-\xi)\left|h_{1}^{1}-k_{1}^{1}\right|\right] \\
& \leq c \sup _{r \leq R_{\varepsilon}^{1}} 24 C_{1, \varepsilon}^{4 \frac{\gamma+\xi-1}{\gamma}} \gamma^{-\frac{1-\xi}{\gamma}} \varepsilon^{8 \frac{\gamma+\xi-1}{\gamma}}\left(\frac{16}{\left(1+r^{2}\right)^{4}}\right)^{\frac{1-\xi}{\gamma}} \\
& \times r^{4-\delta}\left[\xi r^{\delta}\left\|h_{2}^{1}-k_{2}^{1}\right\|_{\mathcal{C}_{\delta}^{4, \alpha}}+(1-\xi) r^{\mu}\left\|h_{1}^{1}-k_{1}^{1}\right\|_{\mathcal{C}_{\mu}^{4, \alpha}}\right] .
\end{aligned}
$$

We conclude that

$$
\left\|\mathcal{M}_{1}\left(h_{1}^{1}, h_{2}^{1}\right)-\mathcal{M}_{1}\left(k_{1}^{1}, k_{2}^{1}\right)\right\|_{\mathcal{C}_{\delta}^{4, \alpha}\left(\mathbb{R}^{4}\right)} \leq c_{\kappa} r_{\varepsilon}^{2}\left\|\left(h_{1}^{1}, h_{2}^{1}\right)-\left(k_{1}^{1}, k_{2}^{1}\right)\right\|_{\mathcal{C}_{\mu}^{4, \alpha}\left(\mathbb{R}^{4}\right) \times \mathcal{C}_{\delta}^{4, \alpha}\left(\mathbb{R}^{4}\right)}
$$

Reducing $\varepsilon_{\kappa}$ if necessary, we can assume that $c_{\kappa} r_{\varepsilon}^{2}<1 / 2$ for all $\varepsilon \in\left(0, \varepsilon_{\kappa}\right)$. There exists also $\gamma_{0} \in(0,1)$ such that $c_{\kappa}(1-\gamma) \leq 1 / 2$ for all $\gamma \in\left(\gamma_{0}, 1\right)$. Therefore (3.15) and (3.16) are enough to show that

$$
\left(h_{1}^{1}, h_{2}^{1}\right) \mapsto\left(\mathcal{N}_{1}\left(h_{1}^{1}, h_{2}^{1}\right), \mathcal{M}_{1}\left(h_{1}^{1}, h_{2}^{1}\right)\right)
$$

is a contraction from the ball

$$
\left\{\left(h_{1}^{1}, h_{2}^{1}\right) \in \mathcal{C}_{\mu}^{4, \alpha}\left(\mathbb{R}^{4}\right) \times \mathcal{C}_{\delta}^{4, \alpha}\left(\mathbb{R}^{4}\right):\left\|\left(h_{1}^{1}, h_{2}^{1}\right)\right\|_{\mathcal{C}_{\mu}^{4, \alpha}\left(\mathbb{R}^{4}\right) \times \mathcal{C}_{\delta}^{4, \alpha}\left(\mathbb{R}^{4}\right)} \leq 2 c_{\kappa} r_{\varepsilon}^{2}\right\}
$$


into itself. Then applying a contraction mapping argument, we obtain the following proposition.

Proposition 3.10. Given $\kappa>0, \mu \in(1,2)$ and $\delta \in\left(0, \min \left\{\left(\frac{\gamma+\xi-1}{\gamma}\right),\left(\frac{\gamma+\xi-1}{\xi}\right)\right\}\right)$, there exist $\varepsilon_{\kappa}>0, c_{\kappa}>0$ and $\gamma_{0} \in(0,1)$ such that for all $\varepsilon \in\left(0, \varepsilon_{\kappa}\right), \gamma \in\left(\gamma_{0}, 1\right)$, for all $\tau_{1}$ in some fixed compact subset of $\left[\tau_{1}^{-}, \tau_{1}^{+}\right] \subset(0, \infty)$ and for $\varphi_{j}^{1}$ and $\psi_{j}^{1}$ satisfying (3.5) and (3.13), there exists a unique $\left(h_{1}^{1}, h_{2}^{1}\right)\left(:=\left(h_{1, \varepsilon, \tau_{1}, \varphi_{1}^{1}, \psi_{1}^{1}}, h_{2, \varepsilon, \tau_{1}, \varphi_{2}^{1}, \psi_{2}^{1}}\right)\right)$ solution of 3.12 such that

$$
\left\|\left(h_{1}^{1}, h_{2}^{1}\right)\right\|_{C_{\mu}^{4, \alpha}\left(\mathbb{R}^{4}\right) \times C_{\delta}^{4, \alpha}\left(\mathbb{R}^{4}\right)} \leq 2 c_{\kappa} r_{\varepsilon}^{2} .
$$

Hence

$$
\begin{aligned}
v_{1}(x):= & \frac{1}{\gamma} \bar{u}\left(x-x^{1}\right)-\frac{1-\gamma}{\gamma} G\left(\frac{\varepsilon x}{\tau_{1}}, x^{2}\right)-\frac{1-\gamma}{\gamma \xi} G\left(\frac{\varepsilon x}{\tau_{1}}, x^{3}\right) \\
& -\frac{\ln \gamma}{\gamma}+h_{1}^{1}(x)+H^{\operatorname{int}}\left(\varphi_{1}^{1}, \psi_{1}^{1} ; \frac{x-x^{1}}{R_{\varepsilon}^{1}}\right), \\
v_{2}(x):= & \frac{1}{\xi} G\left(\frac{\varepsilon x}{\tau_{1}}, x^{3}\right)+G\left(\frac{\varepsilon x}{\tau_{1}}, x^{2}\right)+h_{2}^{1}(x)+H^{\text {int }}\left(\varphi_{2}^{1}, \psi_{2}^{1} ; \frac{x-x^{1}}{R_{\varepsilon}^{1}}\right)
\end{aligned}
$$

solves 3.8 in $B_{R_{\varepsilon}^{1}}\left(x^{1}\right)$.

In $B_{R_{\varepsilon}^{3}}\left(x^{3}\right)$, following the same arguments as the first case by reversing the roles of the functions $u_{1}$ and $u_{2}$ and by respecting the changes of the coefficients we can prove that there exists $\left(h_{1}^{3}, h_{2}^{3}\right) \in \mathcal{C}_{\delta}^{4, \alpha}\left(\mathbb{R}^{4}\right) \times \mathcal{C}_{\mu}^{4, \alpha}\left(\mathbb{R}^{4}\right)$ such that

$$
\left\|\left(h_{1}^{3}, h_{2}^{3}\right)\right\|_{\mathcal{C}_{\delta}^{4, \alpha}\left(\mathbb{R}^{4}\right) \times \mathcal{C}_{\mu}^{4, \alpha}\left(\mathbb{R}^{4}\right)} \leq 2 c_{\kappa} r_{\varepsilon}^{2} .
$$

Furthermore $\left(h_{1}^{3}, h_{2}^{3}\right)$ solves the equations

$$
\begin{aligned}
\Delta^{2} h_{1}^{3}= & \frac{24 C_{3, \varepsilon}^{4 \frac{\gamma+\xi-1}{\xi}} 16^{\frac{1-\gamma}{\xi}} \varepsilon^{8 \frac{\gamma+\xi-1}{\xi}}}{\xi^{\frac{1-\gamma}{\xi}}\left(1+r^{2}\right)^{4 \frac{1-\gamma}{\xi}}} \\
& \times e^{\frac{\gamma+\xi-1}{\xi} G\left(\frac{\varepsilon x}{\tau_{3}}, x^{2}\right)+\frac{\gamma+\xi-1}{\gamma \xi} G\left(\frac{\varepsilon x}{\tau_{3}}, x^{1}\right)+\gamma\left(h_{1}^{3}+H_{\varphi_{1}^{3}, \psi_{1}^{3}}^{\mathrm{int}}\right)+(1-\gamma)\left(h_{2}^{3}+H_{\varphi_{2}^{3}, \psi_{2}^{3}}^{\mathrm{int}}\right)}, \\
\mathbb{L} h_{2}^{3}= & \frac{384}{\xi\left(1+r^{2}\right)^{4}}\left[e^{\xi\left(h_{2}^{3}+H_{\varphi_{2}^{3}, \psi_{2}^{3}}^{\mathrm{int}}\right)+(1-\xi)\left(h_{1}^{3}+H_{\varphi_{1}^{3}, \psi_{1}^{3}}^{\mathrm{int}}\right)}-\xi h_{2}^{3}-1\right] .
\end{aligned}
$$

Given $\kappa>0$ (whose value will be fixed later on), we further assume that the functions $\varphi_{j}^{3}$ and $\psi_{j}^{3}$ satisfy

$$
\left\|\varphi_{j}^{3}\right\|_{\mathcal{C}^{4, \alpha}\left(S^{3}\right)} \leq \kappa r_{\varepsilon}^{2} \quad \text { and } \quad\left\|\psi_{j}^{3}\right\|_{\mathcal{C}^{2, \alpha}\left(S^{3}\right)} \leq \kappa r_{\varepsilon}^{2} \quad \text { for } j=1,2
$$

Then we have the following proposition. 
Proposition 3.11. Given $\kappa>0, \mu \in(1,2)$ and $\delta \in\left(0, \min \left\{\left(\frac{\gamma+\xi-1}{\gamma}\right),\left(\frac{\gamma+\xi-1}{\xi}\right)\right\}\right)$, there exist $\varepsilon_{\kappa}>0, c_{\kappa}>0$ and $\xi_{0} \in(0,1)$ such that for all $\varepsilon \in\left(0, \varepsilon_{\kappa}\right), \xi \in\left(\xi_{0}, 1\right)$, for all $\tau_{3}$ in some fixed compact subset of $\left[\tau_{3}^{-}, \tau_{3}^{+}\right] \subset(0, \infty)$ and for $\varphi_{j}^{3}$ and $\psi_{j}^{3}$ satisfying (3.5) and

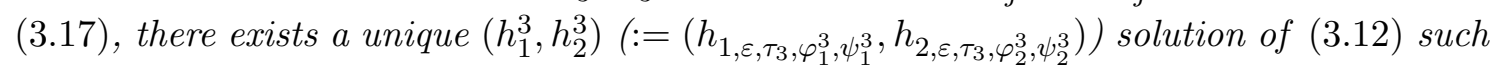
that

$$
\left\|\left(h_{1}^{3}, h_{2}^{3}\right)\right\|_{C_{\delta}^{4, \alpha}\left(\mathbb{R}^{4}\right) \times C_{\mu}^{4, \alpha}\left(\mathbb{R}^{4}\right)} \leq 2 c_{\kappa} r_{\varepsilon}^{2} .
$$

Hence

$$
\begin{aligned}
v_{1}(x):= & \frac{1}{\gamma} G\left(\frac{\varepsilon x}{\tau_{3}}, x^{1}\right)+G\left(\frac{\varepsilon x}{\tau_{3}}, x^{2}\right)+h_{1}^{3}(x)+H^{\text {int }}\left(\varphi_{1}^{3}, \psi_{1}^{3}, \frac{x-x^{3}}{R_{\varepsilon}^{3}}\right), \\
v_{2}(x):= & \frac{1}{\xi} \bar{u}\left(x-x^{3}\right)-\frac{1-\xi}{\xi} G\left(\frac{\varepsilon x}{\tau_{3}}, x^{2}\right)-\frac{1-\xi}{\gamma \xi} G\left(\frac{\varepsilon x}{\tau_{3}}, x^{1}\right) \\
& -\frac{\ln \xi}{\xi}+h_{2}^{3}(x)+H^{\text {int }}\left(\varphi_{2}^{3}, \psi_{2}^{3}, \frac{x-x^{3}}{R_{\varepsilon}^{3}}\right)
\end{aligned}
$$

solves 3.10 in $B_{R_{\varepsilon}^{3}}\left(x^{3}\right)$.

In $B_{R_{\varepsilon}^{2}}\left(x^{2}\right)$, we look for a solution of 3.9 of the form

$$
\begin{aligned}
& v_{1}(x)=\bar{u}\left(x-x^{2}\right)+H^{\mathrm{int}}\left(\varphi_{1}^{2}, \psi_{1}^{2} ; \frac{x-x^{2}}{R_{\varepsilon}^{2}}\right)+h_{1}^{2}(x), \\
& v_{2}(x)=\bar{u}\left(x-x^{2}\right)+H^{\mathrm{int}}\left(\varphi_{2}^{2}, \psi_{2}^{2} ; \frac{x-x^{2}}{R_{\varepsilon}^{2}}\right)+h_{2}^{2}(x) .
\end{aligned}
$$

This amounts to solve the equations

$$
\begin{aligned}
& \mathbb{L} h_{1}^{2}=\frac{384}{\left(1+r^{2}\right)^{4}}\left[e^{\gamma\left(h_{1}^{2}+H_{\varphi_{1}^{2}, \psi_{1}^{2}}^{\mathrm{int}}\right)+(1-\gamma)\left(h_{2}^{2}+H_{\varphi_{2}^{2}, \psi_{2}^{2}}^{\mathrm{int}}\right)}-h_{1}^{2}-1\right], \\
& \mathbb{L} h_{2}^{2}=\frac{384}{\left(1+r^{2}\right)^{4}}\left[e^{\xi\left(h_{2}^{2}+H_{\varphi_{2}^{2}, \psi_{2}^{2}}^{\mathrm{int}}\right)+(1-\xi)\left(h_{1}^{2}+H_{\varphi_{1}^{2}, \psi_{1}^{2}}^{\mathrm{int}}\right)}-h_{2}^{2}-1\right] .
\end{aligned}
$$

We denote by

$$
\mathbb{L} h_{1}^{2}=\mathcal{T}_{3}\left(h_{1}^{2}, h_{2}^{2}\right) \quad \text { and } \quad \mathbb{L} h_{2}^{2}=\mathcal{T}_{4}\left(h_{1}^{2}, h_{2}^{2}\right) .
$$

To find a solution of (3.18), it is enough to find a fixed point $\left(h_{1}^{2}, h_{2}^{2}\right)$ in a small ball of $\mathcal{C}_{\mu}^{4, \alpha}\left(\mathbb{R}^{4}\right) \times \mathcal{C}_{\mu}^{4, \alpha}\left(\mathbb{R}^{4}\right)$, solutions of

$$
\begin{aligned}
& h_{1}^{2}=\mathcal{G}_{\mu} \circ \xi_{\mu, R_{\varepsilon}^{2}} \circ \mathcal{T}_{3}\left(h_{1}^{2}, h_{2}^{2}\right)=\mathcal{N}_{2}\left(h_{1}^{2}, h_{2}^{2}\right), \\
& h_{2}^{2}=\mathcal{G}_{\mu} \circ \xi_{\mu, R_{\varepsilon}^{2}} \circ \mathcal{T}_{4}\left(h_{1}^{2}, h_{2}^{2}\right)=\mathcal{M}_{2}\left(h_{1}^{2}, h_{2}^{2}\right) .
\end{aligned}
$$

Given $\kappa>0$ (whose value will be fixed later on), we further assume that the functions $\varphi_{j}^{2}$ and $\psi_{j}^{2}$ satisfy

$$
\left\|\varphi_{j}^{2}\right\|_{\mathcal{C}^{4, \alpha}\left(S^{3}\right)} \leq \kappa r_{\varepsilon}^{2} \quad \text { and } \quad\left\|\psi_{j}^{2}\right\|_{\mathcal{C}^{2, \alpha}\left(S^{3}\right)} \leq \kappa r_{\varepsilon}^{2} \quad \text { for } j=1,2 .
$$

Then, we have the following result. 
Lemma 3.12. Let $\mu \in(1,2), \gamma_{0}$ and $\xi_{0} \in(0,1)$. Given $\kappa>0$, there exist $\varepsilon_{\kappa}>0$ and $c_{\kappa}>0$ such that for all $\varepsilon \in\left(0, \varepsilon_{\kappa}\right), \gamma \in\left(\gamma_{0}, 1\right)$ and $\xi \in\left(\xi_{0}, 1\right)$. We have

$$
\begin{gathered}
\left\|\mathcal{N}_{2}(0,0)\right\|_{\mathcal{C}_{\mu}^{4, \alpha}\left(\mathbb{R}^{4}\right)} \leq c_{\kappa} r_{\varepsilon}^{2}, \quad\left\|\mathcal{M}_{2}(0,0)\right\|_{\mathcal{C}_{\mu}^{4, \alpha}\left(\mathbb{R}^{4}\right)} \leq c_{\kappa} r_{\varepsilon}^{2} \\
\left\|\mathcal{N}_{2}\left(h_{1}^{2}, h_{2}^{2}\right)-\mathcal{N}_{2}\left(k_{1}^{2}, k_{2}^{2}\right)\right\|_{\mathcal{C}_{\mu}^{4, \alpha}\left(\mathbb{R}^{4}\right)} \leq c_{\kappa}(1-\gamma)\left\|\left(h_{1}^{2}, h_{2}^{2}\right)-\left(k_{1}^{2}, k_{2}^{2}\right)\right\|_{\mathcal{C}_{\mu}^{4, \alpha}\left(\mathbb{R}^{4}\right) \times \mathcal{C}_{\mu}^{4, \alpha}\left(\mathbb{R}^{4}\right)} \\
\left\|\mathcal{M}_{2}\left(h_{1}^{2}, h_{2}^{2}\right)-\mathcal{M}_{2}\left(k_{1}^{2}, k_{2}^{2}\right)\right\|_{\mathcal{C}_{\mu}^{4, \alpha}\left(\mathbb{R}^{4}\right)} \leq c_{\kappa}(1-\xi)\left\|\left(h_{1}^{2}, h_{2}^{2}\right)-\left(k_{1}^{2}, k_{2}^{2}\right)\right\|_{\mathcal{C}_{\mu}^{4, \alpha}\left(\mathbb{R}^{4}\right) \times \mathcal{C}_{\mu}^{4, \alpha}\left(\mathbb{R}^{4}\right)},
\end{gathered}
$$

provided $\left(h_{1}^{2}, h_{2}^{2}\right),\left(k_{1}^{2}, k_{2}^{2}\right)$ in $\mathcal{C}_{\mu}^{4, \alpha}\left(\mathbb{R}^{4}\right) \times \mathcal{C}_{\mu}^{4, \alpha}\left(\mathbb{R}^{4}\right)$ satisfying

$$
\left\|\left(h_{1}^{2}, h_{2}^{2}\right)\right\|_{\mathcal{C}_{\mu}^{4, \alpha}\left(\mathbb{R}^{4}\right) \times \mathcal{C}_{\mu}^{4, \alpha}\left(\mathbb{R}^{4}\right)} \leq 2 c_{\kappa} r_{\varepsilon}^{2} \quad \text { and } \quad\left\|\left(k_{1}^{2}, k_{2}^{2}\right)\right\|_{\mathcal{C}_{\mu}^{4, \alpha}\left(\mathbb{R}^{4}\right) \times \mathcal{C}_{\mu}^{4, \alpha}\left(\mathbb{R}^{4}\right)} \leq 2 c_{\kappa} r_{\varepsilon}^{2}
$$

Proof. The proof of the first and the second estimates follows from the asymptotic behavior of $H^{\text {int }}$ together with the assumption on the norms of $\varphi_{j}^{2}$ and $\psi_{j}^{2}$ given by 3.20 and it follows from the estimate of $H^{\text {int }}$, given by Lemma 3.7, that

$$
\left\|H_{\varphi_{j}^{2}, \psi_{j}^{2}}^{\mathrm{int}}\left(\frac{r}{R_{\varepsilon}^{2}} \cdot\right)\right\|_{\mathcal{C}^{4, \alpha}\left(\bar{B}_{2}(0)-B_{1}(0)\right)} \leq C r^{2}\left(R_{\varepsilon}^{2}\right)^{-2}\left(\left\|\varphi_{j}^{2}\right\|_{\mathcal{C}^{4, \alpha}\left(S^{3}\right)}+\left\|\psi_{j}^{2}\right\|_{\mathcal{C}^{2, \alpha}\left(S^{3}\right)}\right)
$$

for all $r \leq R_{\varepsilon}^{2} / 2$. Then by 3.20 , we get

$$
\left\|H_{\varphi_{j}^{2}, \psi_{j}^{2}}^{\mathrm{int}}\left(\frac{r}{R_{\varepsilon}^{2}} \cdot\right)\right\|_{\mathcal{C}^{4, \alpha}\left(\bar{B}_{2}(0)-B_{1}(0)\right)} \leq c_{\kappa} \varepsilon^{2} r^{2} .
$$

On the other hand,

$$
\begin{aligned}
\sup _{r \leq R_{\varepsilon}^{2}} r^{4-\mu}\left|\mathcal{T}_{3}(0,0)\right| & \leq \sup _{r \leq R_{\varepsilon}^{2}} \frac{384 r^{4-\mu}}{\left(1+r^{2}\right)^{4}} \mid e^{\gamma H_{\varphi_{1}^{2}, \psi_{1}^{2}}^{\mathrm{int}}+(1-\gamma) H_{\varphi_{2}^{2}, \psi_{2}^{2}}^{\mathrm{int}}-1 \mid} \\
& \leq \sup _{r \leq R_{\varepsilon}^{2}} \frac{384 r^{4-\mu}}{\left(1+r^{2}\right)^{4}}\left(\gamma r^{2}\left\|H_{\varphi_{1}^{2}, \psi_{1}^{2}}^{\mathrm{int}}\right\|_{\mathcal{C}_{2}^{4, \alpha}}+(1-\gamma) r^{2}\left\|H_{\varphi_{2}^{2}, \psi_{2}^{2}}^{\mathrm{int}}\right\|_{\mathcal{C}_{2}^{4, \alpha}}\right) .
\end{aligned}
$$

Making use of Proposition 3.2 together with (3.4), for $\mu \in(1,2)$, we get that there exists $c_{\kappa}>0$ such that

$$
\left\|\mathcal{N}_{2}(0,0)\right\|_{\mathcal{C}_{\mu}^{4, \alpha}\left(\mathbb{R}^{4}\right)} \leq c_{\kappa} r_{\varepsilon}^{2}
$$

For the second estimate, we use the same techniques to prove

$$
\left\|\mathcal{M}_{2}(0,0)\right\|_{\mathcal{C}_{\mu}^{4, \alpha}\left(\mathbb{R}^{4}\right)} \leq c_{\kappa} r_{\varepsilon}^{2}
$$

To derive the third estimate, for $\left(h_{1}^{2}, h_{2}^{2}\right),\left(k_{1}^{2}, k_{2}^{2}\right)$ verifying (3.21), we have

$$
\begin{aligned}
& \sup _{r \leq R_{\varepsilon}^{2}} r^{4-\mu}\left|\mathcal{T}_{3}\left(h_{1}^{2}, h_{2}^{2}\right)-\mathcal{T}_{3}\left(k_{1}^{2}, k_{2}^{2}\right)\right| \\
\leq & \sup _{r \leq R_{\varepsilon}^{2}} \frac{384 r^{4-\mu}}{\left(1+r^{2}\right)^{4}} \mid\left(e^{\left.\gamma h_{1}^{2}+\gamma H_{\varphi_{1}^{2}, \psi_{1}^{2}}^{\mathrm{int}}+(1-\gamma) h_{2}^{2}+(1-\gamma) H_{\varphi_{2}^{2}, \psi_{2}^{2}}^{\mathrm{int}}-h_{1}^{2}\right)}\right.
\end{aligned}
$$




$$
\begin{gathered}
-\left(e^{\left.\gamma k_{1}^{2}+\gamma H_{\varphi_{1}^{2}, \psi_{1}^{2}}^{\mathrm{int}}+(1-\gamma) k_{2}^{2}+(1-\gamma) H_{\varphi_{2}^{2}, \psi_{2}^{2}}^{\mathrm{int}}-k_{1}^{2}\right) \mid}\right. \\
\leq c \sup _{r \leq R_{\varepsilon}^{2}} \frac{384 r^{4-\mu}}{\left(1+r^{2}\right)^{4}}\left|(\gamma-1)\left(h_{1}^{2}-k_{1}^{2}\right)+(1-\gamma)\left(h_{2}^{2}-k_{2}^{2}\right)\right| \\
\leq c \sup _{r \leq R_{\varepsilon}^{2}} \frac{384 r^{4-\mu}}{\left(1+r^{2}\right)^{4}}(1-\gamma)\left[r^{\mu}\left\|h_{1}^{2}-k_{1}^{2}\right\|_{\mathcal{C}_{\mu}^{4, \alpha}}+r^{\mu}\left\|h_{2}^{2}-k_{2}^{2}\right\|_{\mathcal{C}_{\mu}^{4, \alpha}}\right] .
\end{gathered}
$$

We conclude that

$$
\left\|\mathcal{N}_{2}\left(h_{1}^{2}, h_{2}^{2}\right)-\mathcal{N}_{2}\left(k_{1}^{2}, k_{2}^{2}\right)\right\|_{\mathcal{C}_{\mu}^{4, \alpha}\left(\mathbb{R}^{4}\right)} \leq c_{\kappa}(1-\gamma)\left\|\left(h_{1}^{2}, h_{2}^{2}\right)-\left(k_{1}^{2}, k_{2}^{2}\right)\right\|_{\mathcal{C}_{\mu}^{4, \alpha}\left(\mathbb{R}^{4}\right) \times \mathcal{C}_{\mu}^{4, \alpha}\left(\mathbb{R}^{4}\right)}
$$

Similarly, we get

$$
\left\|\mathcal{M}_{2}\left(h_{1}^{2}, h_{2}^{2}\right)-\mathcal{M}_{2}\left(k_{1}^{2}, k_{2}^{2}\right)\right\|_{\mathcal{C}_{\mu}^{4, \alpha}\left(\mathbb{R}^{4}\right)} \leq c_{\kappa}(1-\xi)\left\|\left(h_{1}^{2}, h_{2}^{2}\right)-\left(k_{1}^{2}, k_{2}^{2}\right)\right\|_{\mathcal{C}_{\mu}^{4, \alpha}\left(\mathbb{R}^{4}\right) \times \mathcal{C}_{\mu}^{4, \alpha}\left(\mathbb{R}^{4}\right)}
$$

Then there exist $\gamma_{0}$ and $\xi_{0} \in(0,1)$ such that $c_{\kappa}(1-\gamma) \leq 1 / 2$ and $c_{\kappa}(1-\xi) \leq 1 / 2$ for all $\gamma \in\left(\gamma_{0}, 1\right)$ and $\xi \in\left(\xi_{0}, 1\right)$. Therefore $(3.22)$ and $(3.23)$ are enough to show that

$$
\left(h_{1}^{2}, h_{2}^{2}\right) \mapsto\left(\mathcal{N}_{2}\left(h_{1}^{2}, h_{2}^{2}\right), \mathcal{M}_{2}\left(h_{1}^{2}, h_{2}^{2}\right)\right)
$$

is a contraction from the ball

$$
\left\{\left(h_{1}^{2}, h_{2}^{2}\right) \in \mathcal{C}_{\mu}^{4, \alpha}\left(\mathbb{R}^{4}\right) \times \mathcal{C}_{\mu}^{4, \alpha}\left(\mathbb{R}^{4}\right):\left\|\left(h_{1}^{2}, h_{2}^{2}\right)\right\|_{\mathcal{C}_{\mu}^{4, \alpha}\left(\mathbb{R}^{4}\right) \times \mathcal{C}_{\mu}^{4, \alpha}\left(\mathbb{R}^{4}\right)} \leq 2 c_{\kappa} r_{\varepsilon}^{2}\right\}
$$

into itself. Then applying a contraction mapping argument, we obtain the following proposition.

Proposition 3.13. Given $\kappa>0, \mu \in(1,2), \gamma_{0} \in(0,1)$ and $\xi_{0} \in(0,1)$, there exist $\varepsilon_{\kappa}>0$ and $c_{\kappa}>0$ such that for all $\varepsilon \in\left(0, \varepsilon_{\kappa}\right), \gamma \in\left(\gamma_{0}, 1\right)$ and $\xi \in\left(\xi_{0}, 1\right)$, for all $\tau_{2}$ in some fixed compact subset of $\left[\tau_{2}^{-}, \tau_{2}^{+}\right] \subset(0, \infty)$ and for $\varphi_{j}^{2}$ and $\psi_{j}^{2}$ satisfying (3.5) and (3.20), there exists a unique $\left(h_{1}^{2}, h_{2}^{2}\right)\left(:=\left(h_{1, \varepsilon, \tau_{2}, \varphi_{1}^{2}, \psi_{1}^{2}}, h_{2, \varepsilon, \tau_{2}, \varphi_{2}^{2}, \psi_{2}^{2}}\right)\right)$ solution of 3.19 such that

$$
\left\|\left(h_{1}^{2}, h_{2}^{2}\right)\right\|_{C_{\mu}^{4, \alpha}\left(\mathbb{R}^{4}\right) \times C_{\mu}^{4, \alpha}\left(\mathbb{R}^{4}\right)} \leq 2 c_{\kappa} r_{\varepsilon}^{2} .
$$

Hence

$$
\begin{aligned}
& v_{1}(x):=\bar{u}\left(x-x^{2}\right)+h_{1}^{2}(x)+H^{\mathrm{int}}\left(\varphi_{1}^{2}, \psi_{1}^{2} ; \frac{x-x^{2}}{R_{\varepsilon}^{2}}\right), \\
& v_{2}(x):=\bar{u}\left(x-x^{2}\right)+h_{2}^{2}(x)+H^{\mathrm{int}}\left(\varphi_{2}^{2}, \psi_{2}^{2} ; \frac{x-x^{2}}{R_{\varepsilon}^{2}}\right)
\end{aligned}
$$

solves 3.9 in $B_{R_{\varepsilon}^{2}}\left(x^{2}\right)$.

Remark also that the functions $\left(h_{1}^{i}, h_{2}^{i}\right)\left(:=\left(h_{1, \varepsilon, \tau_{i}, \varphi_{1}^{i}, \psi_{1}^{i}}^{i}, h_{2, \varepsilon, \tau_{i}, \varphi_{2}^{i}, \psi_{2}^{i}}^{i}\right)\right)$, for $i \in\{1,2,3\}$, depend continuously on the parameter $\tau_{i}$. 


\subsection{The nonlinear exterior problem}

Given $\widetilde{\boldsymbol{x}}:=\left(\widetilde{x}^{1}, \widetilde{x}^{2}, \widetilde{x}^{3}\right) \in \Omega^{3}$ close to $\boldsymbol{x}:=\left(x^{1}, x^{2}, x^{3}\right), \boldsymbol{\lambda}:=\left(\lambda_{1}, \lambda_{2}, \lambda_{3}\right) \in \mathbb{R}^{3}$ close to 0 , $\widetilde{\varphi}_{1}:=\left(\widetilde{\varphi}_{1}^{1}, \widetilde{\varphi}_{1}^{2}, \widetilde{\varphi}_{1}^{3}\right) \in\left(\mathcal{C}^{4, \alpha}\left(S^{3}\right)\right)^{3}, \widetilde{\varphi}_{2}:=\left(\widetilde{\varphi}_{2}^{1}, \widetilde{\varphi}_{2}^{2}, \widetilde{\varphi}_{2}^{3}\right) \in\left(\mathcal{C}^{4, \alpha}\left(S^{3}\right)\right)^{3}, \widetilde{\boldsymbol{\psi}}_{1}:=\left(\widetilde{\psi}_{1}^{1}, \widetilde{\psi}_{1}^{2}, \widetilde{\psi}_{1}^{3}\right) \in$ $\left(\mathcal{C}^{2, \alpha}\left(S^{3}\right)\right)^{3}$ and $\widetilde{\boldsymbol{\psi}}_{2}:=\left(\widetilde{\psi}_{2}^{1}, \widetilde{\psi}_{2}^{2}, \widetilde{\psi}_{2}^{3}\right) \in\left(\mathcal{C}^{2, \alpha}\left(S^{3}\right)\right)^{3}$ satisfying (3.6). Let $\widetilde{\boldsymbol{w}}_{1}$ and $\widetilde{\boldsymbol{w}}_{2}$ be defined by

$$
\widetilde{\boldsymbol{w}}_{1}(x):=\frac{1+\lambda_{1}}{\gamma} G\left(x, \widetilde{x}^{1}\right)+\left(1+\lambda_{2}\right) G\left(x, \widetilde{x}^{2}\right)+\sum_{i=1}^{3} \chi_{r_{0}}\left(x-\widetilde{x}^{i}\right) H^{\operatorname{ext}}\left(\widetilde{\varphi}_{1}^{i}, \widetilde{\psi}_{1}^{i} ; \frac{x-\widetilde{x}^{i}}{r_{\varepsilon}}\right)
$$

and

$$
\widetilde{\boldsymbol{w}}_{2}(x):=\frac{1+\lambda_{3}}{\xi} G\left(x, \widetilde{x}^{3}\right)+\left(1+\lambda_{2}\right) G\left(x, \widetilde{x}^{2}\right)+\sum_{i=1}^{3} \chi_{r_{0}}\left(x-\widetilde{x}^{i}\right) H^{\operatorname{ext}}\left(\widetilde{\varphi}_{2}^{i}, \widetilde{\psi}_{2}^{i} ; \frac{x-\widetilde{x}^{i}}{r_{\varepsilon}}\right) .
$$

Here $\chi_{r_{0}}$ is a cut-off function identically equal to 1 in $B_{r_{0} / 2}(0)$ and identically equal to 0 outside $B_{r_{0}}(0)$. We would like to find a solution of the system

$$
\Delta^{2} u_{1}=\rho^{4} e^{\gamma u_{1}+(1-\gamma) u_{2}} \quad \text { and } \quad \Delta^{2} u_{2}=\rho^{4} e^{\xi u_{2}+(1-\xi) u_{1}}
$$

in the domain $\bar{\Omega}_{r_{\varepsilon}}(\widetilde{x})$ with $u_{k}=\widetilde{\boldsymbol{w}}_{k}+\widetilde{v}_{k}$ a perturbation of $\widetilde{\boldsymbol{w}}_{k}, k=1,2$. This amounts to solve in $\bar{\Omega}_{r_{\varepsilon}}(\widetilde{x})$,

$\Delta^{2} \widetilde{v}_{1}=\rho^{4} e^{\gamma\left(\widetilde{\boldsymbol{w}}_{1}+\widetilde{v}_{1}\right)+(1-\gamma)\left(\widetilde{\boldsymbol{w}}_{2}+\widetilde{v}_{2}\right)}-\Delta^{2} \widetilde{\boldsymbol{w}}_{1} \quad$ and $\quad \Delta^{2} \widetilde{v}_{2}=\rho^{4} e^{\xi\left(\widetilde{\boldsymbol{w}}_{2}+\widetilde{v}_{2}\right)+(1-\xi)\left(\widetilde{\boldsymbol{w}}_{1}+\widetilde{v}_{1}\right)}-\Delta^{2} \widetilde{\boldsymbol{w}}_{2}$.

For all $\sigma \in\left(0, r_{0} / 2\right)$ and all $\widetilde{\boldsymbol{x}}=\left(\widetilde{x}^{1}, \widetilde{x}^{2}, \widetilde{x}^{3}\right) \in \Omega^{3}$ such that $\|\boldsymbol{x}-\widetilde{\boldsymbol{x}}\| \leq r_{0} / 2$, where $\boldsymbol{x}=\left(x^{1}, x^{2}, x^{3}\right)$, we denote by $\widetilde{\xi}_{\sigma, \widetilde{\boldsymbol{x}}}: \mathcal{C}_{\nu}^{0, \alpha}\left(\bar{\Omega}_{\sigma}(\widetilde{\boldsymbol{x}})\right) \rightarrow \mathcal{C}_{\nu}^{0, \alpha}\left(\bar{\Omega}^{*}(\widetilde{\boldsymbol{x}})\right)$ the extension operator defined by

$$
\begin{cases}\widetilde{\xi}_{\sigma, \widetilde{\boldsymbol{x}}}(f) \equiv f & \text { in } \bar{\Omega}_{\sigma}(\widetilde{\boldsymbol{x}}), \\ \widetilde{\xi}_{\sigma, \widetilde{\boldsymbol{x}}}(f)\left(\widetilde{x}^{j}+x\right)=\widetilde{\chi}\left(\frac{|x|}{\sigma}\right) f\left(\widetilde{x}^{j}+\sigma \frac{x}{|x|}\right) & \text { in } B_{\sigma}\left(\widetilde{x}^{j}\right)-B_{\sigma / 2}\left(\widetilde{x}^{j}\right), \forall 1 \leq j \leq 3, \\ \widetilde{\xi}_{\sigma, \widetilde{\boldsymbol{x}}}(f) \equiv 0 & \text { in } B_{\sigma / 2}\left(\widetilde{x}^{1}\right) \cup B_{\sigma / 2}\left(\widetilde{x}^{2}\right) \cup B_{\sigma / 2}\left(\widetilde{x}^{3}\right) .\end{cases}
$$

Here $\tilde{\chi}$ is a cut-off function over $\mathbb{R}_{+}$which is equal to 1 for $t \geq 1$ and equal to 0 for $t \leq 1 / 2$. Obviously, there exists a constant $\bar{c}=\bar{c}(\nu)>0$ only depending on $\nu$ such that

$$
\left\|\widetilde{\xi}_{\sigma, \widetilde{\boldsymbol{x}}}(w)\right\|_{\mathcal{C}_{\nu}^{0, \alpha}\left(\bar{\Omega}^{*}(\widetilde{\boldsymbol{x}})\right)} \leq \bar{c}\|w\|_{\mathcal{C}_{\nu}^{0, \alpha}\left(\bar{\Omega}_{\sigma}(\widetilde{\boldsymbol{x}})\right)}
$$

We fix $\nu \in(-1,0)$, to solve $(3.25)$, it is enough to find $\left(\widetilde{v}_{1}, \widetilde{v}_{2}\right) \in\left(\mathcal{C}_{\nu}^{4, \alpha}\left(\bar{\Omega}^{*}(\widetilde{\boldsymbol{x}})\right)\right)^{2}$ solution of

$$
\widetilde{v}_{1}=\widetilde{\mathcal{K}}_{\nu} \circ \widetilde{\xi}_{r_{\varepsilon}, \widetilde{\boldsymbol{x}}} \circ \widetilde{S}_{1}\left(\widetilde{v}_{1}, \widetilde{v}_{2}\right) \quad \text { and } \quad \widetilde{v}_{2}=\widetilde{\mathcal{K}}_{\nu} \circ \widetilde{\xi}_{r_{\varepsilon}, \widetilde{\boldsymbol{x}}} \circ \widetilde{S}_{2}\left(\widetilde{v}_{1}, \widetilde{v}_{2}\right) \text {, }
$$


where

$$
\widetilde{S}_{1}\left(\widetilde{v}_{1}, \widetilde{v}_{2}\right)=\rho^{4} e^{\gamma\left(\widetilde{\boldsymbol{w}}_{1}+\widetilde{v}_{1}\right)+(1-\gamma)\left(\widetilde{\boldsymbol{w}}_{2}+\widetilde{v}_{2}\right)}-\Delta^{2} \widetilde{\boldsymbol{w}}_{1}
$$

and

$$
\widetilde{S}_{2}\left(\widetilde{v}_{1}, \widetilde{v}_{2}\right)=\rho^{4} e^{\xi\left(\widetilde{\boldsymbol{w}}_{2}+\widetilde{v}_{2}\right)+(1-\xi)\left(\widetilde{\boldsymbol{w}}_{1}+\widetilde{v}_{1}\right)}-\Delta^{2} \widetilde{\boldsymbol{w}}_{2}
$$

We denote by

$$
\tilde{\mathcal{N}}\left(\widetilde{v}_{1}, \widetilde{v}_{2}\right)=\widetilde{\mathcal{K}}_{\nu} \circ \widetilde{\xi}_{r_{\varepsilon}, \widetilde{\boldsymbol{x}}} \circ \widetilde{S}_{1}\left(\widetilde{v}_{1}, \widetilde{v}_{2}\right) \quad \text { and } \quad \widetilde{\mathcal{M}}\left(\widetilde{v}_{1}, \widetilde{v}_{2}\right)=\widetilde{\mathcal{K}}_{\nu} \circ \widetilde{\xi}_{r_{\varepsilon}, \widetilde{\boldsymbol{x}}} \circ \widetilde{S}_{2}\left(\widetilde{v}_{1}, \widetilde{v}_{2}\right)
$$

Given $\kappa>0$ (whose value will be fixed later on), we further assume that for $i \in\{1,2,3\}$ and $j \in\{1,2\}$ the functions $\widetilde{\varphi}_{j}^{i}, \widetilde{\psi}_{j}^{i}$, the parameters $\lambda_{i}$ and the point $\widetilde{\boldsymbol{x}}=\left(\widetilde{x}^{1}, \widetilde{x}^{2}, \widetilde{x}^{3}\right)$ satisfy

$$
\begin{gathered}
\left\|\widetilde{\varphi}_{j}^{i}\right\|_{\mathcal{C}^{4, \alpha}\left(S^{3}\right)} \leq \kappa r_{\varepsilon}^{2}, \quad\left\|\widetilde{\psi}_{j}^{i}\right\|_{\mathcal{C}^{2, \alpha}\left(S^{3}\right)} \leq \kappa r_{\varepsilon}^{2}, \\
\left|\lambda_{i}\right| \leq \kappa r_{\varepsilon}^{2}, \quad\left|\widetilde{x}^{i}-x^{i}\right| \leq \kappa r_{\varepsilon}
\end{gathered}
$$

Then the following result holds.

Lemma 3.14. Under the above assumptions, there exists a constant $c_{\kappa}>0$ such that

$$
\begin{gathered}
\|\widetilde{\mathcal{N}}(0,0)\|_{\mathcal{C}_{\nu}^{4, \alpha}\left(\bar{\Omega}^{*}(\widetilde{\boldsymbol{x}})\right)} \leq c_{\kappa} r_{\varepsilon}^{2}, \quad\|\widetilde{\mathcal{M}}(0,0)\|_{\mathcal{C}_{\nu}^{4, \alpha}\left(\bar{\Omega}^{*}(\widetilde{\boldsymbol{x}})\right)} \leq c_{\kappa} r_{\varepsilon}^{2}, \\
\left\|\widetilde{\mathcal{N}}\left(\widetilde{v}_{1}, \widetilde{v}_{2}\right)-\tilde{\mathcal{N}}\left(\widetilde{v}_{1}^{\prime}, \widetilde{v}_{2}^{\prime}\right)\right\|_{\mathcal{C}_{\nu}^{4, \alpha}\left(\bar{\Omega}^{*}(\widetilde{\boldsymbol{x}})\right)} \leq c_{\kappa} r_{\varepsilon}^{2}\left\|\left(\widetilde{v}_{1}, \widetilde{v}_{2}\right)-\left(\widetilde{v}_{1}^{\prime}, \widetilde{v}_{2}^{\prime}\right)\right\|_{\left(\mathcal{C}_{\nu}^{4, \alpha}\left(\bar{\Omega}^{*}(\widetilde{\boldsymbol{x}})\right)\right)^{2}}
\end{gathered}
$$

and

$$
\left\|\widetilde{\mathcal{M}}\left(\widetilde{v}_{1}, \widetilde{v}_{2}\right)-\widetilde{\mathcal{M}}\left(\widetilde{v}_{1}^{\prime}, \widetilde{v}_{2}^{\prime}\right)\right\|_{\mathcal{C}_{\nu}^{4, \alpha}\left(\bar{\Omega}^{*}(\widetilde{\boldsymbol{x}})\right)} \leq c_{\kappa} r_{\varepsilon}^{2}\left\|\left(\widetilde{v}_{1}, \widetilde{v}_{2}\right)-\left(\widetilde{v}_{1}^{\prime}, \widetilde{v}_{2}^{\prime}\right)\right\|_{\left(\mathcal{C}_{\nu}^{4, \alpha}\left(\bar{\Omega}^{*}(\widetilde{\boldsymbol{x}})\right)\right)^{2}},
$$

provided $\left(\widetilde{v}_{1}, \widetilde{v}_{2}, \widetilde{v}_{1}^{\prime}, \widetilde{v}_{2}^{\prime}\right) \in\left(\mathcal{C}_{\nu}^{4, \alpha}\left(\bar{\Omega}^{*}(\widetilde{\boldsymbol{x}})\right)\right)^{4}$ satisfy

$$
\left\|\left(\widetilde{v}_{1}, \widetilde{v}_{2}\right)\right\|_{\left(\mathcal{C}_{\nu}^{4, \alpha}\left(\bar{\Omega}^{*}(\widetilde{\boldsymbol{x}})\right)\right)^{2}} \leq 2 c_{\kappa} r_{\varepsilon}^{2} \quad \text { and } \quad\left\|\left(\widetilde{v}_{1}^{\prime}, \widetilde{v}_{2}^{\prime}\right)\right\|_{\left(\mathcal{C}_{\nu}^{4, \alpha}\left(\bar{\Omega}^{*}(\widetilde{\boldsymbol{x}})\right)\right)^{2}} \leq 2 c_{\kappa} r_{\varepsilon}^{2}
$$

Proof. As for the interior problem, the proof of the two first estimates follows from the asymptotic behavior of $H^{\text {ext }}$ together with the assumption on the norm of boundary data $\widetilde{\varphi}_{j}^{i}$ and $\widetilde{\psi}_{j}^{i}$ given by (3.28). Indeed, let $c_{\kappa}$ be a constant depending only on $\kappa$, by Lemma 3.7 .

$$
\left|H^{\operatorname{ext}}\left(\widetilde{\varphi}_{j}^{i}, \widetilde{\psi}_{j}^{i} ; \frac{x-\widetilde{x}^{i}}{r_{\varepsilon}}\right)\right| \leq c_{\kappa} r_{\varepsilon}^{3} r^{-1} .
$$

On the other hand,

$$
\widetilde{S}_{1}(0,0)=\rho^{4} e^{\gamma \widetilde{\boldsymbol{w}}_{1}+(1-\gamma) \widetilde{\boldsymbol{w}}_{2}}-\Delta^{2} \widetilde{\boldsymbol{w}}_{1} \quad \text { and } \quad \widetilde{S}_{2}(0,0)=\rho^{4} e^{\xi \widetilde{\boldsymbol{w}}_{2}+(1-\xi) \widetilde{\boldsymbol{w}}_{1}}-\Delta^{2} \widetilde{\boldsymbol{w}}_{2}
$$

We will estimate $\widetilde{S}_{1}(0,0)$ in different subregions of $\bar{\Omega}^{*}(\widetilde{\boldsymbol{x}})$. 
- In $B_{r_{0} / 2}\left(\widetilde{x}^{1}\right)-B_{r_{\varepsilon}}\left(\widetilde{x}^{1}\right)$, we have $\chi_{r_{0}}\left(x-\widetilde{x}^{1}\right)=1, \chi_{r_{0}}\left(x-\widetilde{x}^{2}\right)=0, \chi_{r_{0}}\left(x-\widetilde{x}^{3}\right)=0$ and $\Delta^{2} \widetilde{\boldsymbol{w}}_{1}=0$, so that $\left|\widetilde{S}_{1}(0,0)\right|=\rho^{4} e^{\gamma \widetilde{w}_{1}+(1-\gamma) \widetilde{w}_{2}}$. Then

$$
\left|\widetilde{S}_{1}(0,0)\right| \leq c_{\kappa} \varepsilon^{4}\left|x-\widetilde{x}^{1}\right|^{-8\left(1+\lambda_{1}\right)} \leq c_{\kappa} \varepsilon^{4} r^{-8\left(1+\lambda_{1}\right)} \text {. }
$$

Hence, for $\nu \in(-1,0)$ and $\lambda_{1}$ small enough, we get

$$
\left\|\widetilde{S}_{1}(0,0)\right\|_{\mathcal{C}_{\nu-4}^{0, \alpha}\left(B_{r_{0} / 2}\left(\widetilde{x}^{1}\right)\right)} \leq \sup _{r_{\varepsilon} \leq r \leq r_{0} / 2} r^{4-\nu}\left|\widetilde{S}_{1}(0,0)\right| \leq c_{\kappa} \varepsilon^{4} r_{\varepsilon}^{-4}
$$

- In $B_{r_{0}}\left(\widetilde{x}^{1}\right)-B_{r_{0} / 2}\left(\widetilde{x}^{1}\right)$, using the estimate 3.31 , then we have

$$
\begin{aligned}
\left|\widetilde{S}_{1}(0,0)\right| & \leq c_{\kappa} \varepsilon^{4} r^{-8\left(1+\lambda_{1}\right)}+\left|\left[\Delta^{2}, \chi_{r_{0}}\left(x-\widetilde{x}^{1}\right)\right] H^{\operatorname{ext}}\left(\widetilde{\varphi}_{1}^{1}, \widetilde{\psi}_{1}^{1} ; \frac{x-\widetilde{x}^{1}}{r_{\varepsilon}}\right)\right| \\
& \leq c_{\kappa}\left(\varepsilon^{4} r^{-8\left(1+\lambda_{1}\right)}+r^{-1} r_{\varepsilon}^{3}\right),
\end{aligned}
$$

where

$$
\begin{aligned}
{\left[\Delta^{2}, \chi_{r_{0}}\right] w=} & w \Delta^{2} \chi_{r_{0}}+2 \Delta w \Delta \chi_{r_{0}}+4 \nabla(\Delta w) \cdot \nabla \chi_{r_{0}} \\
& +4 \nabla w \cdot \nabla\left(\Delta \chi_{r_{0}}\right)+4 \sum_{i, j=1}^{4} \frac{\partial^{2} \chi_{r_{0}}}{\partial x_{i} \partial x_{j}} \frac{\partial^{2} w}{\partial x_{i} \partial x_{j}}
\end{aligned}
$$

Hence, for $\nu \in(-1,0)$ and $\lambda_{1}$ small enough, we get

$$
\left\|\widetilde{S}_{1}(0,0)\right\|_{\mathcal{C}_{\nu-4}^{0, \alpha}\left(B_{r_{0}}\left(\widetilde{x}^{1}\right)-B_{r_{0} / 2}\left(\widetilde{x}^{1}\right)\right)} \leq \sup _{r_{0} / 2 \leq r \leq r_{0}} r^{4-\nu}\left|\widetilde{S}_{1}(0,0)\right| \leq c_{\kappa} r_{\varepsilon}^{2}
$$

- In $B_{r_{0} / 2}\left(\widetilde{x}^{2}\right)-B_{r_{\varepsilon}}\left(\widetilde{x}^{2}\right)$, we have $\chi_{r_{0}}\left(x-\widetilde{x}^{1}\right)=0, \chi_{r_{0}}\left(x-\widetilde{x}^{2}\right)=1, \chi_{r_{0}}\left(x-\widetilde{x}^{3}\right)=0$ and $\Delta^{2} \widetilde{\boldsymbol{w}}_{1}=0$, so that $\widetilde{S}_{1}(0,0)=\rho^{4} e^{\gamma \widetilde{\boldsymbol{w}}_{1}+(1-\gamma) \widetilde{\boldsymbol{w}}_{2}}$. Then

$$
\left|\widetilde{S}_{1}(0,0)\right| \leq c_{\kappa} \varepsilon^{4}\left|x-\widetilde{x}^{2}\right|^{-8\left(1+\lambda_{2}\right)} \leq c_{\kappa} \varepsilon^{4} r^{-8\left(1+\lambda_{2}\right)} .
$$

Hence, for $\nu \in(-1,0)$ and $\lambda_{2}$ small enough, we get

$$
\left\|\widetilde{S}_{1}(0,0)\right\|_{\mathcal{C}_{\nu-4}^{0, \alpha}\left(B_{r_{0}}\left(\widetilde{x}^{2}\right)\right)} \leq \sup _{r_{\varepsilon} \leq r \leq r_{0} / 2} r^{4-\nu}\left|\widetilde{S}_{1}(0,0)\right| \leq c_{\kappa} r_{\varepsilon}^{2}
$$

- In $B_{r_{0}}\left(\widetilde{x}^{2}\right)-B_{r_{0} / 2}\left(\widetilde{x}^{2}\right)$, using the estimate 3.31 , there holds

$$
\begin{aligned}
\left|\widetilde{S}_{1}(0,0)\right| & \leq c_{\kappa} \varepsilon^{4} r^{-8\left(1+\lambda_{2}\right)}+\left|\left[\Delta^{2}, \chi_{r_{0}}\left(x-\widetilde{x}^{2}\right)\right] H^{\operatorname{ext}}\left(\widetilde{\varphi}_{1}^{2}, \widetilde{\psi}_{1}^{2} ; \frac{x-\widetilde{x}^{2}}{r_{\varepsilon}}\right)\right| \\
& \leq c_{\kappa}\left(\varepsilon^{4} r^{-8\left(1+\lambda_{2}\right)}+r^{-1} r_{\varepsilon}^{3}\right) .
\end{aligned}
$$

Hence, for $\nu \in(-1,0)$ and $\lambda_{2}$ small enough, we get

$$
\left\|\widetilde{S}_{1}(0,0)\right\|_{\mathcal{C}_{\nu-4}^{0, \alpha}\left(B_{r_{0}}\left(\widetilde{x}^{2}\right)\right)} \leq \sup _{r_{0} / 2 \leq r \leq r_{0}} r^{4-\nu}\left|\widetilde{S}_{1}(0,0)\right| \leq c_{\kappa} r_{\varepsilon}^{2}
$$


Similarly, for $\nu \in(-1,0)$ and $\lambda_{3}$ small enough, we can prove the same result for $\widetilde{x}^{3}$.

- In $\Omega-\left(B_{r_{0}}\left(\widetilde{x}^{1}\right) \cup B_{r_{0}}\left(\widetilde{x}^{2}\right) \cup B_{r_{0}}\left(\widetilde{x}^{3}\right)\right)$, we have $\chi_{r_{0}}\left(x-\widetilde{x}^{1}\right)=0, \chi_{r_{0}}\left(x-\widetilde{x}^{2}\right)=0$, $\chi_{r_{0}}\left(x-\widetilde{x}^{3}\right)=0$ and $\Delta^{2} \widetilde{\boldsymbol{w}}_{1}=0$. So for $\nu \in(-1,0)$, we have

$$
\left\|\widetilde{S}_{1}(0,0)\right\|_{\mathcal{C}_{\nu-4}^{0, \alpha}\left(\bar{\Omega}-\bigcup_{i=1}^{3} B_{r_{0}}\left(\widetilde{x}^{i}\right)\right)} \leq \sup _{r \geq r_{0}} r^{4-\nu}\left|\widetilde{S}_{1}(0,0)\right| \leq c_{\kappa} \varepsilon^{4}
$$

We conclude that

$$
\left\|\widetilde{S}_{1}(0,0)\right\|_{\mathcal{C}_{\nu-4}^{0, \alpha}\left(\bar{\Omega}_{r_{0}}(\widetilde{\boldsymbol{x}})\right)} \leq c_{\kappa} r_{\varepsilon}^{4}
$$

Now, we are interested in the second equation of the previous system.

- In $B_{r_{0} / 2}\left(\widetilde{x}^{1}\right)-B_{r_{\varepsilon}}\left(\widetilde{x}^{1}\right)$, we have $\chi_{r_{0}}\left(x-\widetilde{x}^{1}\right)=1, \chi_{r_{0}}\left(x-\widetilde{x}^{2}\right)=0, \chi_{r_{0}}\left(x-\widetilde{x}^{3}\right)=0$ and $\Delta^{2} \widetilde{\boldsymbol{w}}_{1}=0$, so that $\left|\widetilde{S}_{2}(0,0)\right|=\rho^{4} e^{\xi \widetilde{w}_{2}+(1-\xi) \widetilde{w}_{1}}$. Then

$$
\left|\widetilde{S}_{2}(0,0)\right| \leq c_{\kappa} \varepsilon^{4}\left|x-\widetilde{x}^{1}\right|^{-8 \frac{(1-\xi)\left(1+\lambda_{1}\right)}{\gamma}} \leq c_{\kappa} \varepsilon^{4} r^{-8 \frac{(1-\xi)\left(1+\lambda_{1}\right)}{\gamma}} .
$$

Hence, for $\nu \in(-1,0)$ and $\lambda_{1}$ small enough, we get

$$
\left\|\widetilde{S}_{2}(0,0)\right\|_{\mathcal{C}_{\nu-4}^{0, \alpha}\left(B_{r_{0} / 2}\left(\widetilde{x}^{1}\right)\right)} \leq \sup _{r_{\varepsilon} \leq r \leq r_{0} / 2} r^{4-\nu}\left|\widetilde{S}_{2}(0,0)\right| \leq c_{\kappa} r_{\varepsilon}^{2} .
$$

- In $B_{r_{0}}\left(\widetilde{x}^{1}\right)-B_{r_{0} / 2}\left(\widetilde{x}^{1}\right)$, using the estimate 3.31 , then we have

$$
\begin{aligned}
\left|\widetilde{S}_{2}(0,0)\right| & \leq c_{\kappa} \varepsilon^{4} r^{-8 \frac{(1-\xi)\left(1+\lambda_{1}\right)}{\gamma}}+\left|\left[\Delta^{2}, \chi_{r_{0}}\left(x-\widetilde{x}^{1}\right)\right] H^{\operatorname{ext}}\left(\widetilde{\varphi}_{2}^{1}, \widetilde{\psi}_{2}^{1} ; \frac{x-\widetilde{x}^{1}}{r_{\varepsilon}}\right)\right| \\
& \leq c_{\kappa}\left(\varepsilon^{4} r^{-8 \frac{(1-\xi)\left(1+\lambda_{1}\right)}{\gamma}}+r^{-1} r_{\varepsilon}^{3}\right)
\end{aligned}
$$

where

$$
\begin{aligned}
{\left[\Delta^{2}, \chi_{r_{0}}\right] w=} & w \Delta^{2} \chi_{r_{0}}+2 \Delta w \Delta \chi_{r_{0}}+4 \nabla(\Delta w) \cdot \nabla \chi_{r_{0}} \\
& +4 \nabla w \cdot \nabla\left(\Delta \chi_{r_{0}}\right)+4 \sum_{i, j=1}^{4} \frac{\partial^{2} \chi_{r_{0}}}{\partial x_{i} \partial x_{j}} \frac{\partial^{2} w}{\partial x_{i} \partial x_{j}}
\end{aligned}
$$

Hence, for $\nu \in(-1,0)$ and $\lambda_{1}$ small enough, we get

$$
\left\|\widetilde{S}_{2}(0,0)\right\|_{\mathcal{C}_{\nu-4}^{0, \alpha}\left(B_{r_{0}}\left(\widetilde{x}^{1}\right)-B_{r_{0} / 2}\left(\widetilde{x}^{1}\right)\right)} \leq \sup _{r_{0} / 2 \leq r \leq r_{0}} r^{4-\nu}\left|\widetilde{S}_{2}(0,0)\right| \leq c_{\kappa} r_{\varepsilon}^{2}
$$

- In $B_{r_{0} / 2}\left(\widetilde{x}^{2}\right)-B_{r_{\varepsilon}}\left(\widetilde{x}^{2}\right)$, we have $\chi_{r_{0}}\left(x-\widetilde{x}^{1}\right)=0, \chi_{r_{0}}\left(x-\widetilde{x}^{2}\right)=1, \chi_{r_{0}}\left(x-\widetilde{x}^{3}\right)=0$ and $\Delta^{2} \widetilde{\boldsymbol{w}}_{2}=0$, so that $\widetilde{S}_{2}(0,0)=\rho^{4} e^{\xi \widetilde{\boldsymbol{w}}_{2}+(1-\xi) \widetilde{\boldsymbol{w}}_{1}}$. Then

$$
\left|\widetilde{S}_{2}(0,0)\right| \leq c_{\kappa} \varepsilon^{4}\left|x-\widetilde{x}^{2}\right|^{-8\left(1+\lambda_{2}\right)} \leq c_{\kappa} \varepsilon^{4} r^{-8\left(1+\lambda_{2}\right)} .
$$

Hence, for $\nu \in(-1,0)$ and $\lambda_{2}$ small enough, we get

$$
\left\|\widetilde{S}_{2}(0,0)\right\|_{\mathcal{C}_{\nu-4}^{0, \alpha}\left(B_{r_{0}}\left(\widetilde{x}^{2}\right)\right)} \leq \sup _{r_{\varepsilon} \leq r \leq r_{0} / 2} r^{4-\nu}\left|\widetilde{S}_{2}(0,0)\right| \leq c_{\kappa} r_{\varepsilon}^{2}
$$


- In $B_{r_{0}}\left(\widetilde{x}^{2}\right)-B_{r_{0} / 2}\left(\widetilde{x}^{2}\right)$, using the estimate 3.31 , there holds

$$
\begin{aligned}
\left|\widetilde{S}_{2}(0,0)\right| & \leq c_{\kappa} \varepsilon^{4} r^{-8\left(1+\lambda_{2}\right)}+\left|\left[\Delta^{2}, \chi_{r_{0}}\left(x-\widetilde{x}^{2}\right)\right] H^{\mathrm{ext}}\left(\widetilde{\varphi}_{2}^{2}, \widetilde{\psi}_{2}^{2} ; \frac{x-\widetilde{x}^{2}}{r_{\varepsilon}}\right)\right| \\
& \leq c_{\kappa}\left(\varepsilon^{4} r^{-8\left(1+\lambda_{2}\right)}+r^{-1} r_{\varepsilon}^{3}\right) .
\end{aligned}
$$

Hence, for $\nu \in(-1,0)$ and $\lambda_{2}$ small enough, we get

$$
\left\|\widetilde{S}_{2}(0,0)\right\|_{\mathcal{C}_{\nu-4}^{0, \alpha}\left(B_{r_{0}}\left(\widetilde{x}^{2}\right)\right)} \leq \sup _{r_{0} / 2 \leq r \leq r_{0}} r^{4-\nu}\left|\widetilde{S}_{2}(0,0)\right| \leq c_{\kappa} r_{\varepsilon}^{2}
$$

Similarly, for $\nu \in(-1,0)$ and $\lambda_{3}$ small enough, we can prove the same result for $\widetilde{x}^{3}$.

- In $\Omega-\left(B_{r_{0}}\left(\widetilde{x}^{1}\right) \cup B_{r_{0}}\left(\widetilde{x}^{2}\right) \cup B_{r_{0}}\left(\widetilde{x}^{3}\right)\right)$, we have $\chi_{r_{0}}\left(x-\widetilde{x}^{1}\right)=0, \chi_{r_{0}}\left(x-\widetilde{x}^{2}\right)=0$, $\chi_{r_{0}}\left(x-\widetilde{x}^{3}\right)=0$ and $\Delta^{2} \widetilde{\boldsymbol{w}}_{1}=0$. So for $\nu \in(-1,0)$, we have

$$
\left\|\widetilde{S}_{2}(0,0)\right\|_{\mathcal{C}_{\nu-4}^{0, \alpha}\left(\bar{\Omega}-\bigcup_{i=1}^{3} B_{r_{0}}\left(\widetilde{x}^{i}\right)\right)} \leq \sup _{r \geq r_{0}} r^{4-\nu}\left|\widetilde{S}_{2}(0,0)\right| \leq c_{\kappa} \varepsilon^{4} .
$$

Making use of Proposition 3.5 together with (3.26) we conclude that

$$
\|\widetilde{\mathcal{N}}(0,0)\|_{\mathcal{C}_{\nu}^{4, \alpha}\left(\bar{\Omega}^{*}(\widetilde{\boldsymbol{x}})\right)} \leq c_{\kappa} r_{\varepsilon}^{2} \quad \text { and } \quad\|\widetilde{\mathcal{M}}(0,0)\|_{\mathcal{C}_{\nu}^{4, \alpha}\left(\bar{\Omega}^{*}(\widetilde{\boldsymbol{x}})\right)} \leq c_{\kappa} r_{\varepsilon}^{2}
$$

For the proof of the third estimate, let $\widetilde{v}_{1}, \widetilde{v}_{2}, \widetilde{v}_{1}^{\prime}$ and $\widetilde{v}_{2}^{\prime} \in \mathcal{C}_{\nu}^{4, \alpha}\left(\bar{\Omega}^{*}\right)$ satisfy $(3.30)$, we have

$$
\begin{aligned}
\left|\widetilde{S}_{1}\left(\widetilde{v}_{1}, \widetilde{v}_{2}\right)-\widetilde{S}_{1}\left(\widetilde{v}_{1}^{\prime}, \widetilde{v}_{2}^{\prime}\right)\right| & \leq c_{\kappa} \varepsilon^{4} e^{\gamma \widetilde{w}_{1}+(1-\gamma) \widetilde{w}_{2}}\left|e^{\gamma \widetilde{v}_{1}+(1-\gamma) \widetilde{v}_{2}}-e^{\gamma \widetilde{v}_{1}^{\prime}+(1-\gamma) \widetilde{v}_{2}^{\prime}}\right| \\
& \leq c_{\kappa} \varepsilon^{4}\left(\gamma\left|\widetilde{v}_{1}-\widetilde{v}_{1}^{\prime}\right|+(1-\gamma)\left|\widetilde{v}_{2}-\widetilde{v}_{2}^{\prime}\right|\right) .
\end{aligned}
$$

So, for $\lambda_{i}, i=1,2,3$, small enough and using the estimate (3.26), there exists $c_{\kappa}$ (depending on $\kappa$ ) such that

$$
\left\|\tilde{\mathcal{N}}\left(\widetilde{v}_{1}, \widetilde{v}_{2}\right)-\tilde{\mathcal{N}}\left(\widetilde{v}_{1}^{\prime}, \widetilde{v}_{2}^{\prime}\right)\right\|_{\mathcal{C}_{\nu}^{4, \alpha}\left(\bar{\Omega}^{*}(\widetilde{\boldsymbol{x}})\right)} \leq c_{\kappa} r_{\varepsilon}^{2}\left(\left\|\widetilde{v}_{1}-\widetilde{v}_{1}^{\prime}\right\|_{C_{\nu}^{4, \alpha}\left(\bar{\Omega}^{*}(\widetilde{\boldsymbol{x}})\right)}+\left\|\widetilde{v}_{2}-\widetilde{v}_{2}^{\prime}\right\|_{C_{\nu}^{4, \alpha}\left(\bar{\Omega}^{*}(\widetilde{\boldsymbol{x}})\right)}\right) .
$$

Similarly we can use the same arguments to prove

$$
\left\|\widetilde{\mathcal{M}}\left(\widetilde{v}_{1}, \widetilde{v}_{2}\right)-\widetilde{\mathcal{M}}\left(\widetilde{v}_{1}^{\prime}, \widetilde{v}_{2}^{\prime}\right)\right\|_{\mathcal{C}_{\nu}^{4, \alpha}\left(\bar{\Omega}^{*}(\widetilde{\boldsymbol{x}})\right)} \leq c_{\kappa} r_{\varepsilon}^{2}\left(\left\|\widetilde{v}_{1}-\widetilde{v}_{1}^{\prime}\right\|_{C_{\nu}^{4, \alpha}\left(\bar{\Omega}^{*}(\widetilde{\boldsymbol{x}})\right)}+\left\|\widetilde{v}_{2}-\widetilde{v}_{2}^{\prime}\right\|_{C_{\nu}^{4, \alpha}\left(\bar{\Omega}^{*}(\widetilde{\boldsymbol{x}})\right)}\right) .
$$

Reducing $\varepsilon_{\kappa}$ if necessary, we can assume that $c_{\kappa} r_{\varepsilon}^{2} \leq 1 / 2$ for all $\varepsilon \in\left(0, \varepsilon_{\kappa}\right)$. Then, (3.32) and (3.33) are enough to show that

$$
\left(\widetilde{v}_{1}, \widetilde{v}_{2}\right) \mapsto\left(\widetilde{\mathcal{N}}\left(\widetilde{v}_{1}, \widetilde{v}_{2}\right), \widetilde{\mathcal{M}}\left(\widetilde{v}_{1}, \widetilde{v}_{2}\right)\right)
$$

is a contraction from the ball

$$
\left\{\left(\widetilde{v}_{1}, \widetilde{v}_{2}\right) \in\left(C_{\nu}^{4, \alpha}\left(\mathbb{R}^{4}\right)\right)^{2}:\left\|\left(\widetilde{v}_{1}, \widetilde{v}_{2}\right)\right\|_{\left(C_{\nu}^{4, \alpha}\left(\mathbb{R}^{4}\right)\right)^{2}} \leq 2 c_{\kappa} r_{\varepsilon}^{2}\right\}
$$

into itself. Hence there exists a unique fixed point $\left(\widetilde{v}_{1}, \widetilde{v}_{2}\right)$ in this set, which is a solution of (3.27). Applying a fixed point Theorem for contraction mappings, we conclude that 
Proposition 3.15. Given $\kappa>0$, there exists $\varepsilon_{\kappa}>0$ (depending on $\kappa$ ) such that for any $\varepsilon \in\left(0, \varepsilon_{\kappa}\right), \lambda_{i}$ and $\widetilde{x}^{i}$ satisfying (3.29) and functions $\widetilde{\varphi}_{j}^{i}$ and $\widetilde{\psi}_{j}^{i}$ satisfying (3.6) and (3.28),

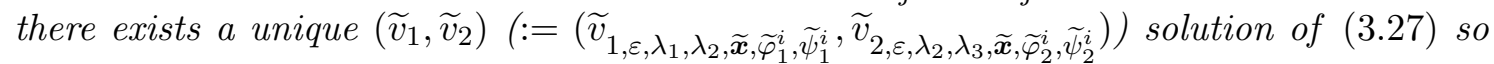
that for $v_{k}(k=1,2)$ defined by

$$
\begin{aligned}
& v_{1}(x):=\frac{1+\lambda_{1}}{\gamma} G\left(x, \widetilde{x}^{1}\right)+\left(1+\lambda_{2}\right) G\left(x, \widetilde{x}^{2}\right)+\sum_{i=1}^{3} \chi_{r_{0}}\left(x-\widetilde{x}^{i}\right) H^{\mathrm{ext}}\left(\widetilde{\varphi}_{1}^{i}, \widetilde{\psi}_{1}^{i} ; \frac{x-\widetilde{x}^{i}}{r_{\varepsilon}}\right)+\widetilde{v}_{1}(x), \\
& v_{2}(x):=\frac{1+\lambda_{3}}{\xi} G\left(x, \widetilde{x}^{3}\right)+\left(1+\lambda_{2}\right) G\left(x, \widetilde{x}^{2}\right)+\sum_{i=1}^{3} \chi_{r_{0}}\left(x-\widetilde{x}^{i}\right) H^{\operatorname{ext}}\left(\widetilde{\varphi}_{2}^{i}, \widetilde{\psi}_{2}^{i} ; \frac{x-\widetilde{x}^{i}}{r_{\varepsilon}}\right)+\widetilde{v}_{2}(x)
\end{aligned}
$$

solves (3.24) in $\bar{\Omega}_{r_{\varepsilon}}(\widetilde{\boldsymbol{x}})$. In addition, we have

$$
\left\|\left(\widetilde{v}_{1}, \widetilde{v}_{2}\right)\right\|_{C_{\nu}^{4, \alpha}\left(\bar{\Omega}^{*}(\widetilde{\boldsymbol{x}})\right)} \leq 2 c_{\kappa} r_{\varepsilon}^{2}
$$

\subsection{The nonlinear Cauchy-data matching}

We will gather the results of the previous sections. Using the previous notations, assume that $\widetilde{\boldsymbol{x}}:=\left(\widetilde{x}^{1}, \widetilde{x}^{2}, \widetilde{x}^{3}\right) \in \Omega^{3}$ are given close to $\boldsymbol{x}:=\left(x^{1}, x^{2}, x^{3}\right)$. Assume also that

$$
\boldsymbol{\tau}:=\left(\tau_{1}, \tau_{2}, \tau_{3}\right) \in\left[\tau_{1}^{-}, \tau_{1}^{+}\right] \times\left[\tau_{2}^{-}, \tau_{2}^{+}\right] \times\left[\tau_{3}^{-}, \tau_{3}^{+}\right] \subset(0, \infty)^{3}
$$

are given (the values of $\tau_{l}^{-}$and $\tau_{l}^{+}$, for $l=1,2,3$ will be fixed later). First, we consider some set of boundary data $\varphi^{i}:=\left(\varphi_{1}^{i}, \varphi_{2}^{i}\right) \in\left(\mathcal{C}^{4, \alpha}\left(S^{3}\right)\right)^{2}$ and $\boldsymbol{\psi}^{i}:=\left(\psi_{1}^{i}, \psi_{2}^{i}\right) \in\left(\mathcal{C}^{2, \alpha}\left(S^{3}\right)\right)^{2}$. Let $\varepsilon \in\left(0, \varepsilon_{\kappa}\right)$ and according to the result of Propositions 3.10, 3.11 and 3.13, we can find, $u_{\text {int }}:=\left(u_{\text {int }, 1}, u_{\text {int }, 2}\right)$ a solution of 3.7 in $B_{r_{\varepsilon}}\left(\widetilde{x}^{1}\right) \cup B_{r_{\varepsilon}}\left(\widetilde{x}^{2}\right) \cup B_{r_{\varepsilon}}\left(\widetilde{x}^{3}\right)$, which can be decomposed as

$$
u_{\mathrm{int}, 1}(x):= \begin{cases}\frac{1}{\gamma} u_{\varepsilon, \tau_{1}}\left(x-\widetilde{x}^{1}\right)-\frac{1-\gamma}{\gamma} G\left(x, \widetilde{x}^{2}\right)-\frac{1-\gamma}{\gamma \xi} G\left(x, \widetilde{x}^{3}\right) & \\ -\frac{\ln \gamma}{\gamma}+H_{\varphi_{1}^{1}, \psi_{1}^{1}}^{\mathrm{int}}\left(\frac{x-\widetilde{x}^{1}}{r_{\varepsilon}}\right)+h_{1}^{1}\left(\frac{R_{\varepsilon}^{1}\left(x-\widetilde{x}^{1}\right)}{r_{\varepsilon}}\right) & \text { in } B_{r_{\varepsilon}}\left(\widetilde{x}^{1}\right), \\ u_{\varepsilon, \tau_{2}}\left(x-\widetilde{x}^{2}\right)+H_{\varphi_{1}^{2}, \psi_{1}^{2}}^{\mathrm{int}}\left(\frac{x-\widetilde{x}^{2}}{r_{\varepsilon}}\right)+h_{1}^{2}\left(\frac{R_{\varepsilon}^{2}\left(x-\widetilde{x}^{2}\right)}{r_{\varepsilon}}\right) & \text { in } B_{r_{\varepsilon}}\left(\widetilde{x}^{2}\right), \\ \frac{1}{\gamma} G\left(x, \widetilde{x}^{1}\right)+G\left(x, \widetilde{x}^{2}\right)+H_{\varphi_{1}^{3}, \psi_{1}^{3}}^{\mathrm{int}}\left(\frac{x-\widetilde{x}^{3}}{r_{\varepsilon}}\right)+h_{1}^{3}\left(\frac{R_{\varepsilon}^{3}\left(x-\widetilde{x}^{3}\right)}{r_{\varepsilon}}\right) & \text { in } B_{r_{\varepsilon}}\left(\widetilde{x}^{3}\right)\end{cases}
$$

and

$$
u_{\mathrm{int}, 2}(x):= \begin{cases}\frac{1}{\xi} G\left(x, \widetilde{x}^{3}\right)+G\left(x, \widetilde{x}^{2}\right)+H_{\varphi_{2}^{1}, \psi_{2}^{1}}^{\mathrm{int}}\left(\frac{x-\widetilde{x}^{1}}{r_{\varepsilon}}\right)+h_{2}^{1}\left(\frac{R_{\varepsilon}^{1}\left(x-\widetilde{x}^{1}\right)}{r_{\varepsilon}}\right) & \text { in } B_{r_{\varepsilon}}\left(\widetilde{x}^{1}\right), \\ u_{\varepsilon, \tau_{2}}\left(x-\widetilde{x}^{2}\right)+H_{\varphi_{2}^{2}, \psi_{2}^{2}}^{\mathrm{int}}\left(\frac{x-\widetilde{x}^{2}}{r_{\varepsilon}}\right)+h_{2}^{2}\left(\frac{R_{\varepsilon}^{2}\left(x-\widetilde{x}^{2}\right)}{r_{\varepsilon}}\right) & \text { in } B_{r_{\varepsilon}}\left(\widetilde{x}^{2}\right), \\ \frac{1}{\xi} u_{\varepsilon, \tau_{3}}\left(x-\widetilde{x}^{3}\right)-\frac{1-\xi}{\xi} G\left(x, \widetilde{x}^{2}\right)-\frac{1-\xi}{\gamma \xi} G\left(x, \widetilde{x}^{1}\right) & \\ -\frac{\ln \xi}{\xi}+H_{\varphi_{2}^{3}, \psi_{2}^{3}}^{\mathrm{int}}\left(\frac{x-\widetilde{x}^{3}}{r_{\varepsilon}}\right)+h_{2}^{3}\left(\frac{R_{\varepsilon}^{3}\left(x-\widetilde{x}^{3}\right)}{r_{\varepsilon}}\right) & \text { in } B_{r_{\varepsilon}}\left(\widetilde{x}^{3}\right),\end{cases}
$$

where for $i \in\{1,2,3\}$ and $j \in\{1,2\}, R_{\varepsilon}^{i}=\tau_{i} \frac{r_{\varepsilon}}{\varepsilon}$ and the functions $h_{j}^{i}$ satisfy

$$
\left\|\left(h_{1}^{1}, h_{2}^{1}\right)\right\|_{\mathcal{C}_{\mu}^{4, \alpha}\left(\mathbb{R}^{4}\right) \times \mathcal{C}_{\delta}^{4, \alpha}\left(\mathbb{R}^{4}\right)} \leq 2 c_{\kappa} r_{\varepsilon}^{2}, \quad\left\|\left(h_{1}^{2}, h_{2}^{2}\right)\right\|_{\left(\mathcal{C}_{\mu}^{4, \alpha}\left(\mathbb{R}^{4}\right)\right)^{2}} \leq 2 c_{\kappa} r_{\varepsilon}^{2}
$$


and

$$
\left\|\left(h_{1}^{3}, h_{2}^{3}\right)\right\|_{\mathcal{C}_{\delta}^{4, \alpha}\left(\mathbb{R}^{4}\right) \times \mathcal{C}_{\mu}^{4, \alpha}\left(\mathbb{R}^{4}\right)} \leq 2 c_{\kappa} r_{\varepsilon}^{2} .
$$

Similarly, given some boundary data $\widetilde{\varphi}_{j}^{i} \in C^{4, \alpha}\left(S^{3}\right), \widetilde{\psi}_{j}^{i} \in C^{2, \alpha}\left(S^{3}\right)$ satisfying (3.6), $\left(\lambda_{1}, \lambda_{2}, \lambda_{3}\right) \in \mathbb{R}^{3}$ satisfying (3.29), provided $\varepsilon \in\left(0, \varepsilon_{\kappa}\right)$, by Proposition 3.15, we find a solution $u_{\text {ext }}:=\left(u_{\text {ext }, 1}, u_{\text {ext }, 2}\right)$ of $(3.7)$ in $\left.\bar{\Omega} \backslash\left(B_{r_{\varepsilon}}\left(\widetilde{x}^{1}\right) \cup B_{r_{\varepsilon}}\left(\widetilde{x}^{2}\right)\right) \cup B_{r_{\varepsilon}}\left(\widetilde{x}^{3}\right)\right)$ which can be decomposed as

$$
\begin{aligned}
u_{\mathrm{ext}, 1}(x):= & \frac{1+\lambda_{1}}{\gamma} G\left(x, \widetilde{x}^{1}\right)+\left(1+\lambda_{2}\right) G\left(x, \widetilde{x}^{2}\right) \\
& +\sum_{i=1}^{3} \chi_{r_{0}}\left(x-\widetilde{x}^{i}\right) H^{\operatorname{ext}}\left(\widetilde{\varphi}_{1}^{i}, \widetilde{\psi}_{1}^{i} ; \frac{x-\widetilde{x}^{i}}{r_{\varepsilon}}\right)+\widetilde{v}_{1}(x), \\
u_{\mathrm{ext}, 2}(x):= & \frac{1+\lambda_{3}}{\xi} G\left(x, \widetilde{x}^{3}\right)+\left(1+\lambda_{2}\right) G\left(x, \widetilde{x}^{2}\right) \\
& +\sum_{i=1}^{3} \chi_{r_{0}}\left(x-\widetilde{x}^{i}\right) H^{\operatorname{ext}}\left(\widetilde{\varphi}_{2}^{i}, \widetilde{\psi}_{2}^{i} ; \frac{x-\widetilde{x}^{i}}{r_{\varepsilon}}\right)+\widetilde{v}_{2}(x),
\end{aligned}
$$

with $\widetilde{v}_{1}, \widetilde{v}_{2} \in C_{\nu}^{4, \alpha}\left(\bar{\Omega}^{*}(\widetilde{\boldsymbol{x}})\right)$ satisfying

$$
\left\|\left(\widetilde{v}_{1}, \widetilde{v}_{2}\right)\right\|_{\left(C_{\nu}^{4, \alpha}\left(\bar{\Omega}^{*}(\widetilde{\boldsymbol{x}})\right)\right)^{2}} \leq 2 c_{\kappa} r_{\varepsilon}^{2} .
$$

It remains to determine the parameters and the boundary data in such a way that the function equal to $u_{\text {int }}$ in $B_{r_{\varepsilon}}\left(\widetilde{x}^{1}\right) \cup B_{r_{\varepsilon}}\left(\widetilde{x}^{2}\right) \cup B_{r_{\varepsilon}}\left(\widetilde{x}^{3}\right)$ and equal to $u_{\text {ext }}$ in $\bar{\Omega}_{r_{\varepsilon}}(\widetilde{\boldsymbol{x}})$ is a smooth function. This amounts to find the boundary data and the parameters so that, for each $j=1,2$,

$$
\begin{array}{rlrl}
u_{\mathrm{int}, j} & =u_{\mathrm{ext}, j}, & \partial_{r} u_{\mathrm{int}, j} & =\partial_{r} u_{\mathrm{ext}, j}, \\
\Delta u_{\mathrm{int}, j} & =\Delta u_{\mathrm{ext}, j}, \quad \partial_{r} \Delta u_{\mathrm{int}, j}=\partial_{r} \Delta u_{\mathrm{ext}, j}
\end{array}
$$

on $\partial B_{r_{\varepsilon}}\left(\widetilde{x}^{1}\right), \partial B_{r_{\varepsilon}}\left(\widetilde{x}^{2}\right)$ and $\partial B_{r_{\varepsilon}}\left(\widetilde{x}^{3}\right)$.

Suppose that (3.34) is verified, this provides that for each $\varepsilon$ small enough $u_{\varepsilon} \in \mathcal{C}^{4, \alpha}$ (which is obtained by patching together the functions $u_{\text {int }}$ and the function $u_{\text {ext }}$ ), a weak solution of our system and elliptic regularity theory implies that this solution is in fact smooth. That will complete the proof since, as $\varepsilon$ tends to 0 , the sequence of solutions we have obtain satisfies the required singular limit behaviors.

Before we proceed, the following remarks are due. First it will be convenient to observe that the function $u_{\varepsilon, \tau_{i}}$ can be expanded as

$$
u_{\varepsilon, \tau_{i}}(x)=-4 \ln \tau_{i}-8 \ln |x|+\mathcal{O}\left(\frac{\varepsilon^{2} \tau_{i}^{-2}}{|x|^{2}}\right) \quad \text { on } \partial B_{r_{\varepsilon}}(0) .
$$


- On $\partial B_{r_{\varepsilon}}\left(\widetilde{x}^{1}\right)$, we have

$$
\begin{aligned}
& \left(u_{\text {int }, 1}-u_{\text {ext }, 1}\right)(x) \\
= & -\frac{4}{\gamma} \ln \tau_{1}+\frac{8 \lambda_{1}}{\gamma} \ln \left|x-\widetilde{x}^{1}\right|-\frac{1-\gamma}{\gamma \xi} G\left(x, \widetilde{x}^{3}\right)-\frac{\ln \gamma}{\gamma} \\
& +h_{1}^{1}\left(R_{\varepsilon}^{1} \frac{x-\widetilde{x}^{1}}{r_{\varepsilon}}\right)+H^{\operatorname{int}}\left(\varphi_{1}^{1}, \psi_{1}^{1} ; \frac{x-\widetilde{x}^{1}}{r_{\varepsilon}}\right)-H^{\operatorname{ext}}\left(\widetilde{\varphi}_{1}^{1}, \widetilde{\psi}_{1}^{1} ; \frac{x-\widetilde{x}^{1}}{r_{\varepsilon}}\right) \\
& -\frac{1+\lambda_{1}}{\gamma} H\left(x, \widetilde{x}^{1}\right)-\left(1+\lambda_{2}+\frac{1-\gamma}{\gamma}\right) G\left(x, \widetilde{x}^{2}\right)+\mathcal{O}\left(\frac{\varepsilon^{2} \tau_{1}^{-2}}{\left|x-\widetilde{x}^{1}\right|^{2}}\right)+\mathcal{O}\left(r_{\varepsilon}^{2}\right) .
\end{aligned}
$$

Next, even though all functions are defined on $\partial B_{r_{\varepsilon}}\left(\widetilde{x}^{1}\right)$ in $(3.34)$, it will be more convenient to solve on $S^{3}$ the following set of equations

$$
\begin{aligned}
& \left(u_{\mathrm{int}, 1}-u_{\mathrm{ext}, 1}\right)\left(\widetilde{x}^{1}+r_{\varepsilon} \cdot\right)=0, \quad \partial_{r}\left(u_{\mathrm{int}, 1}-u_{\mathrm{ext}, 1}\right)\left(\widetilde{x}^{1}+r_{\varepsilon} \cdot\right)=0, \\
& \Delta\left(u_{\text {int }, 1}-u_{\text {ext }, 1}\right)\left(\widetilde{x}^{1}+r_{\varepsilon} \cdot\right)=0, \quad \partial_{r} \Delta\left(u_{\text {int }, 1}-u_{\text {ext }, 1}\right)\left(\widetilde{x}^{1}+r_{\varepsilon} \cdot\right)=0 .
\end{aligned}
$$

Since the boundary data are chosen to satisfy 3.5 or 3.6 . We decompose

$$
\begin{array}{ll}
\varphi_{1}^{1}=\varphi_{1,0}^{1}+\varphi_{1,1}^{1}+\varphi_{1}^{1, \perp}, & \psi_{1}^{1}=8 \varphi_{1,0}^{1}+12 \varphi_{1,1}^{1}+\psi_{1}^{1, \perp}, \\
\widetilde{\varphi}_{1}^{1}=\widetilde{\varphi}_{1,0}^{1}+\widetilde{\varphi}_{1,1}^{1}+\widetilde{\varphi}_{1}^{1, \perp}, & \widetilde{\psi}_{1}^{1}=\widetilde{\psi}_{1,1}^{1}+\widetilde{\psi}_{1}^{1, \perp},
\end{array}
$$

where $\varphi_{1,0}^{1}, \widetilde{\varphi}_{1,0}^{1} \in \mathbb{E}_{0}=\mathbb{R}$ are constant on $S^{3}, \varphi_{1,1}^{1}, \widetilde{\varphi}_{1,1}^{1}, \widetilde{\psi}_{1,1}^{1}$ belong to $\mathbb{E}_{1}=\operatorname{Span}\left\{e_{1}, e_{2}\right.$, $\left.e_{3}, e_{4}\right\}$ and $\varphi_{1}^{1, \perp}, \widetilde{\varphi}_{1}^{1, \perp}, \psi_{1}^{1, \perp}, \widetilde{\psi}_{1}^{1, \perp}$ are $L^{2}\left(S^{3}\right)$ orthogonal to $\mathbb{E}_{0}$ and $\mathbb{E}_{1}$.

Using (3.35), we have for $x \in S^{3}$,

$$
\begin{aligned}
& \left(u_{\text {int }, 1}-u_{\text {ext }, 1}\right)\left(\widetilde{x}^{1}+r_{\varepsilon} x\right) \\
= & -\frac{4}{\gamma} \ln \tau_{1}+\frac{8 \lambda_{1}}{\gamma} \ln \left(r_{\varepsilon}|x|\right)-\frac{1}{\gamma}\left(H\left(\widetilde{x}^{1}, \widetilde{x}^{1}\right)+G\left(\widetilde{x}^{1}, \widetilde{x}^{2}\right)+\frac{1-\gamma}{\xi} G\left(\widetilde{x}^{1}, \widetilde{x}^{3}\right)\right) \\
& +H^{\operatorname{int}}\left(\varphi_{1}^{1}, \psi_{1}^{1} ; x\right)-H^{\operatorname{ext}}\left(\widetilde{\varphi}_{1}^{1}, \widetilde{\psi}_{1}^{1} ; x\right)-\frac{\ln \gamma}{\gamma} \\
& -\frac{\lambda_{1}}{\gamma} H\left(\widetilde{x}^{1}, \widetilde{x}^{1}\right)-\lambda_{2} G\left(\widetilde{x}^{1}, \widetilde{x}^{2}\right)+\mathcal{O}\left(r_{\varepsilon}^{2}\right) .
\end{aligned}
$$

Then, the projection of the equations $(3.36)$ over $\mathbb{E}_{0}$ will yield

$$
\begin{aligned}
-4 \ln \tau_{1}+8 \lambda_{1} \ln r_{\varepsilon}-\ln \gamma+\gamma \varphi_{1,0}^{1}-\gamma \widetilde{\varphi}_{1,0}^{1}-\mathcal{E}_{1}\left(\widetilde{x}^{1}, \widetilde{\boldsymbol{x}}\right)+\mathcal{O}\left(r_{\varepsilon}^{2}\right) & =0 \\
8 \lambda_{1}+2 \gamma \varphi_{1,0}^{1}+2 \gamma \widetilde{\varphi}_{1,0}^{1}+\mathcal{O}\left(r_{\varepsilon}^{2}\right) & =0 \\
16 \lambda_{1}+8 \gamma \varphi_{1,0}^{1}+\mathcal{O}\left(r_{\varepsilon}^{2}\right) & =0 \\
-32 \lambda_{1}+\mathcal{O}\left(r_{\varepsilon}^{2}\right) & =0
\end{aligned}
$$

where

$$
\mathcal{E}_{1}(\cdot, \widetilde{\boldsymbol{x}}):=H\left(\cdot, \widetilde{x}^{1}\right)+G\left(\cdot, \widetilde{x}^{2}\right)+\frac{1-\gamma}{\xi} G\left(\cdot, \widetilde{x}^{3}\right) .
$$


The system 3.37 can be simply written as $\lambda_{1}=\mathcal{O}\left(r_{\varepsilon}^{2}\right), \quad \varphi_{1,0}^{1}=\mathcal{O}\left(r_{\varepsilon}^{2}\right), \quad \widetilde{\varphi}_{1,0}^{1}=\mathcal{O}\left(r_{\varepsilon}^{2}\right) \quad$ and $\quad \frac{1}{\ln r_{\varepsilon}}\left[4 \ln \tau_{1}+\ln \gamma+\mathcal{E}_{1}\left(\widetilde{x}^{1}, \widetilde{\boldsymbol{x}}\right)\right]=\mathcal{O}\left(r_{\varepsilon}^{2}\right)$.

We are now in a position to define $\tau_{1}^{-}$and $\tau_{1}^{+}$. In fact, according to the above analysis, as $\varepsilon$ tends to 0 , we expect that $\widetilde{x}^{i}$ will converge to $x^{i}$ for $i \in\{1,2,3\}$ and $\tau_{1}$ will converge to $\tau_{1}^{*}$ satisfying

$$
4 \ln \tau_{1}^{*}=-\ln \gamma-\mathcal{E}_{1}\left(x^{1}, \boldsymbol{x}\right)
$$

Hence it is enough to choose $\tau_{1}^{-}$and $\tau_{1}^{+}$in such a way that

$$
4 \ln \left(\tau_{1}^{-}\right)<-\ln \gamma-\mathcal{E}_{1}\left(x^{1}, \boldsymbol{x}\right)<4 \ln \left(\tau_{1}^{+}\right)
$$

Consider now the projection of $(3.36)$ over $\mathbb{E}_{1}$. Given a smooth function $f$ defined in $\Omega$, we identify its gradient $\nabla f=\left(\partial_{x_{1}} f, \ldots, \partial_{x_{4}} f\right)$ with the element of $\mathbb{E}_{1}$,

$$
\bar{\nabla} f=\sum_{i=1}^{4} \partial_{x_{i}} f e_{i}
$$

Keeping these notations in mind, we obtain the system of equations

$$
\begin{aligned}
\varphi_{1,1}^{1}-\widetilde{\varphi}_{1,1}^{1}-\bar{\nabla} \mathcal{E}_{1}\left(\widetilde{x}^{1}, \widetilde{\boldsymbol{x}}\right)+\mathcal{O}\left(r_{\varepsilon}^{2}\right) & =0 \\
3 \varphi_{1,1}^{1}+3 \widetilde{\varphi}_{1,1}^{1}+\frac{1}{2} \widetilde{\psi}_{1,1}^{1}-\bar{\nabla} \mathcal{E}_{1}\left(\widetilde{x}^{1}, \widetilde{\boldsymbol{x}}\right)+\mathcal{O}\left(r_{\varepsilon}^{2}\right) & =0 \\
15 \varphi_{1,1}^{1}-3 \widetilde{\varphi}_{1,1}^{1}-\widetilde{\psi}_{1,1}^{1}+\mathcal{O}\left(r_{\varepsilon}^{2}\right) & =0 \\
15 \varphi_{1,1}^{1}+15 \widetilde{\varphi}_{1,1}^{1}+\frac{18}{4} \widetilde{\psi}_{1,1}^{1}+\mathcal{O}\left(r_{\varepsilon}^{2}\right) & =0
\end{aligned}
$$

which can be simplified as follows

$$
\varphi_{1,1}^{1}=\mathcal{O}\left(r_{\varepsilon}^{2}\right), \quad \widetilde{\varphi}_{1,1}^{1}=\mathcal{O}\left(r_{\varepsilon}^{2}\right), \quad \widetilde{\psi}_{1,1}^{1}=\mathcal{O}\left(r_{\varepsilon}^{2}\right) \quad \text { and } \quad \bar{\nabla} \mathcal{E}_{1}\left(\widetilde{x}^{1}, \widetilde{\boldsymbol{x}}\right)=\mathcal{O}\left(r_{\varepsilon}^{2}\right)
$$

Finally, we consider the projection onto $L^{2}\left(S^{3}\right)^{\perp}$. This yields the system

$$
\begin{aligned}
& \varphi_{1}^{1, \perp}-\widetilde{\varphi}_{1}^{1, \perp}+\mathcal{O}\left(r_{\varepsilon}^{2}\right)=0, \partial_{r}\left(H_{\varphi_{1}^{1, \perp}, \psi_{1}^{1, \perp}}^{\mathrm{int}}-H_{\widetilde{\varphi}_{1}^{1, \perp}, \widetilde{\psi}_{1}^{1, \perp}}^{\mathrm{ext}}\right)+\mathcal{O}\left(r_{\varepsilon}^{2}\right)=0 \\
& \psi_{1}^{1, \perp}-\widetilde{\psi}_{1}^{1, \perp}+\mathcal{O}\left(r_{\varepsilon}^{2}\right)=0, \quad \partial_{r} \Delta\left(H_{\varphi_{1}^{1, \perp}, \psi_{1}^{1, \perp}}^{\mathrm{int}}-H_{\widetilde{\varphi}_{1}^{1, \perp}, \widetilde{\psi}_{1}^{1, \perp}}^{\mathrm{ext}}\right)+\mathcal{O}\left(r_{\varepsilon}^{2}\right)=0 .
\end{aligned}
$$

Thanks to the result of Lemma 3.8, this last system can be rewritten as

$$
\varphi_{1}^{1, \perp}=\mathcal{O}\left(r_{\varepsilon}^{2}\right), \quad \widetilde{\varphi}_{1}^{1, \perp}=\mathcal{O}\left(r_{\varepsilon}^{2}\right), \quad \psi_{1}^{1, \perp}=\mathcal{O}\left(r_{\varepsilon}^{2}\right) \quad \text { and } \quad \widetilde{\psi}_{1}^{1, \perp}=\mathcal{O}\left(r_{\varepsilon}^{2}\right) .
$$

If we define the parameter $t_{1} \in \mathbb{R}$ by

$$
t_{1}=\frac{1}{\ln r_{\varepsilon}}\left[4 \ln \tau_{1}+\ln \gamma+\mathcal{E}_{1}\left(\widetilde{x}^{1}, \widetilde{\boldsymbol{x}}\right)\right],
$$


then the systems found by projecting 3.36 gather in this equality

$$
T_{1, \varepsilon}^{1}=\left(t_{1}, \lambda_{1}, \varphi_{1,0}^{1}, \widetilde{\varphi}_{1,0}^{1}, \varphi_{1,1}^{1}, \widetilde{\varphi}_{1,1}^{1}, \widetilde{\psi}_{1,1}^{1}, \bar{\nabla} \mathcal{E}_{1}\left(\widetilde{x}^{1}, \widetilde{\boldsymbol{x}}\right), \varphi_{1}^{1, \perp}, \widetilde{\varphi}_{1}^{1, \perp}, \psi_{1}^{1, \perp}, \widetilde{\psi}_{1}^{1, \perp}\right)=\mathcal{O}\left(r_{\varepsilon}^{2}\right)
$$

As usual, the terms $\mathcal{O}\left(r_{\varepsilon}^{2}\right)$ depend nonlinearly on all the variables on the left side, but is bounded (in the appropriate norm) by a constant (independent of $\varepsilon$ and $\kappa$ ) times $r_{\varepsilon}^{2}$, provided $\varepsilon \in\left(0, \varepsilon_{\kappa}\right)$.

- On $\partial B_{r_{\varepsilon}}\left(\widetilde{x}^{1}\right)$, we have

$$
\begin{aligned}
\left(u_{\mathrm{int}, 2}-u_{\mathrm{ext}, 2}\right)(x)= & -\frac{\lambda_{3}}{\xi} G\left(x, \widetilde{x}^{3}\right)+G\left(x, \widetilde{x}^{2}\right)+h_{2}^{1}\left(R_{\varepsilon}^{1} \frac{x-\widetilde{x}^{1}}{r_{\varepsilon}}\right)+H^{\mathrm{int}}\left(\varphi_{2}^{1}, \psi_{2}^{1} ; \frac{x-\widetilde{x}^{1}}{r_{\varepsilon}}\right) \\
& -\left(1+\lambda_{2}\right) G\left(x, \widetilde{x}^{2}\right)-H^{\operatorname{ext}}\left(\widetilde{\varphi}_{2}^{1}, \widetilde{\psi}_{2}^{1} ; \frac{x-\widetilde{x}^{1}}{r_{\varepsilon}}\right)+\mathcal{O}\left(r_{\varepsilon}^{2}\right) .
\end{aligned}
$$

In the same manner as above, we will solve on $S^{3}$ the following system

$$
\begin{aligned}
& \left(u_{\text {int }, 2}-u_{\text {ext }, 2}\right)\left(\widetilde{x}^{1}+r_{\varepsilon} \cdot\right)=0, \quad \partial_{r}\left(u_{\text {int }, 2}-u_{\text {ext }, 2}\right)\left(\widetilde{x}^{1}+r_{\varepsilon} \cdot\right)=0, \\
& \Delta\left(u_{\text {int }, 2}-u_{\text {ext }, 2}\right)\left(\widetilde{x}^{1}+r_{\varepsilon} \cdot\right)=0, \quad \partial_{r} \Delta\left(u_{\text {int }, 2}-u_{\text {ext }, 2}\right)\left(\widetilde{x}^{1}+r_{\varepsilon} \cdot\right)=0 .
\end{aligned}
$$

We decompose

$$
\begin{array}{ll}
\varphi_{2}^{1}=\varphi_{2,0}^{1}+\varphi_{2,1}^{1}+\varphi_{2}^{1, \perp}, & \psi_{2}^{1}=8 \varphi_{2,0}^{1}+12 \varphi_{2,1}^{1}+\psi_{2}^{1, \perp}, \\
\widetilde{\varphi}_{2}^{1}=\widetilde{\varphi}_{2,0}^{1}+\widetilde{\varphi}_{2,1}^{1}+\widetilde{\varphi}_{2}^{1, \perp}, & \widetilde{\psi}_{2}^{1}=\widetilde{\psi}_{2,1}^{1}+\widetilde{\psi}_{2}^{1, \perp}
\end{array}
$$

with $\varphi_{2,0}^{1}, \widetilde{\varphi}_{2,0}^{1} \in \mathbb{E}_{0}, \varphi_{2,1}^{1}, \widetilde{\varphi}_{2,1}^{1}, \widetilde{\psi}_{2,1}^{1} \in \mathbb{E}_{1}$ and $\varphi_{2}^{1, \perp}, \widetilde{\varphi}_{2}^{1, \perp}, \psi_{2}^{1, \perp}, \widetilde{\psi}_{2}^{1, \perp}$ belong to $\left(L^{2}\left(S^{3}\right)\right)^{\perp}$.

Projecting the set of equations $(3.39)$ over $\mathbb{E}_{0}$, we get

$$
\varphi_{2,0}^{1}-\widetilde{\varphi}_{2,0}^{1}+\mathcal{O}\left(r_{\varepsilon}^{2}\right)=0, \quad 2 \varphi_{2,0}^{1}+2 \widetilde{\varphi}_{2,0}^{1}+\mathcal{O}\left(r_{\varepsilon}^{2}\right)=0, \quad 8 \varphi_{2,0}^{1}+\mathcal{O}\left(r_{\varepsilon}^{2}\right)=0
$$

From the $L^{2}$-projection of 3.39 over $\mathbb{E}_{1}$, we obtain the system of equations

$$
\begin{array}{rr}
\varphi_{2,1}^{1}-\widetilde{\varphi}_{2,1}^{1}+\mathcal{O}\left(r_{\varepsilon}^{2}\right)=0, & 3 \varphi_{2,1}^{1}+3 \widetilde{\varphi}_{2,1}^{1}+\frac{1}{2} \widetilde{\psi}_{2,1}^{1}+\mathcal{O}\left(r_{\varepsilon}^{2}\right)=0, \\
15 \varphi_{2,1}^{1}-3 \widetilde{\varphi}_{2,1}^{1}-\widetilde{\psi}_{2,1}^{1}+\mathcal{O}\left(r_{\varepsilon}^{2}\right)=0, & 15 \varphi_{2,1}^{1}+15 \widetilde{\varphi}_{2,1}^{1}+\frac{18}{4} \widetilde{\psi}_{2,1}^{1}+\mathcal{O}\left(r_{\varepsilon}^{2}\right)=0 .
\end{array}
$$

Finally, we consider the $L^{2}$-projection onto $\left(L^{2}\left(S^{3}\right)\right)^{\perp}$. This yields the system

$$
\begin{aligned}
& \varphi_{2}^{1, \perp}-\widetilde{\varphi}_{2}^{1, \perp}+\mathcal{O}\left(r_{\varepsilon}^{2}\right)=0, \partial_{r}\left(H_{\varphi_{2}^{1, \perp}, \psi_{2}^{1, \perp}}^{\mathrm{int}}-H_{\widetilde{\varphi}_{2}^{1, \perp}, \widetilde{\psi}_{2}^{1, \perp}}^{\mathrm{ext}}\right)+\mathcal{O}\left(r_{\varepsilon}^{2}\right)=0 \\
& \psi_{2}^{1, \perp}-\widetilde{\psi}_{2}^{1, \perp}+\mathcal{O}\left(r_{\varepsilon}^{2}\right)=0, \quad \partial_{r} \Delta\left(H_{\varphi_{2}^{1, \perp}, \psi_{2}^{1, \perp}}^{\mathrm{int}}-H_{\widetilde{\varphi}_{2}^{1, \perp}, \widetilde{\psi}_{2}^{1, \perp}}^{\mathrm{ext}}\right)+\mathcal{O}\left(r_{\varepsilon}^{2}\right)=0 .
\end{aligned}
$$

Using again Lemma 3.8, the above system can be rewritten as

$$
\varphi_{2}^{1, \perp}=\mathcal{O}\left(r_{\varepsilon}^{2}\right), \quad \widetilde{\varphi}_{2}^{1, \perp}=\mathcal{O}\left(r_{\varepsilon}^{2}\right), \quad \psi_{2}^{1, \perp}=\mathcal{O}\left(r_{\varepsilon}^{2}\right) \quad \text { and } \quad \widetilde{\psi}_{2}^{1, \perp}=\mathcal{O}\left(r_{\varepsilon}^{2}\right)
$$


Then the systems found by projecting $(3.39)$ gather in this equality

$$
T_{2, \varepsilon}^{1}=\left(\varphi_{2,0}^{1}, \widetilde{\varphi}_{2,0}^{1}, \varphi_{2,1}^{1}, \widetilde{\varphi}_{2,1}^{1}, \widetilde{\psi}_{2,1}^{1}, \varphi_{2}^{1, \perp}, \widetilde{\varphi}_{2}^{1, \perp}, \psi_{2}^{1, \perp}, \widetilde{\psi}_{2}^{1, \perp}\right)=\mathcal{O}\left(r_{\varepsilon}^{2}\right)
$$

- On $\partial B_{r_{\varepsilon}}\left(\widetilde{x}^{2}\right)$, we have

$$
\begin{aligned}
& (1-\xi)\left(u_{\mathrm{int}, 1}-u_{\mathrm{ext}, 1}\right)(x)+(1-\gamma)\left(u_{\mathrm{int}, 2}-u_{\mathrm{ext}, 2}\right)(x) \\
= & -4(2-\gamma-\xi) \ln \tau_{2}+8(2-\gamma-\xi) \lambda_{2} \ln \left|x-\widetilde{x}^{2}\right| \\
& +(1-\xi) h_{1}^{2}\left(R_{\varepsilon}^{2} \frac{x-\widetilde{x}^{2}}{r_{\varepsilon}}\right)+(1-\gamma) h_{2}^{2}\left(R_{\varepsilon}^{2} \frac{x-\widetilde{x}^{2}}{r_{\varepsilon}}\right) \\
& +(1-\xi) H^{\operatorname{int}}\left(\varphi_{1}^{2}, \psi_{1}^{2} ; \frac{x-\widetilde{x}^{2}}{r_{\varepsilon}}\right)+(1-\gamma) H^{\mathrm{int}}\left(\varphi_{2}^{2}, \psi_{2}^{2} ; \frac{x-\widetilde{x}^{2}}{r_{\varepsilon}}\right) \\
& -(1-\xi) H^{\operatorname{ext}}\left(\widetilde{\varphi}_{1}^{2}, \widetilde{\psi}_{1}^{2} ; \frac{x-\widetilde{x}^{2}}{r_{\varepsilon}}\right)-(1-\gamma) H^{\operatorname{ext}}\left(\widetilde{\varphi}_{2}^{2}, \widetilde{\psi}_{2}^{2} ; \frac{x-\widetilde{x}^{2}}{r_{\varepsilon}}\right) \\
& -\left[(2-\gamma-\xi) H\left(x, \widetilde{x}^{2}\right)+\frac{1-\xi}{\gamma} G\left(x, \widetilde{x}^{1}\right)+\frac{1-\gamma}{\xi} G\left(x, \widetilde{x}^{3}\right)\right]+\mathcal{O}\left(\frac{\varepsilon^{2} \tau_{2}^{-2}}{\left|x-\widetilde{x}^{2}\right|^{2}}\right)+\mathcal{O}\left(r_{\varepsilon}^{2}\right) .
\end{aligned}
$$

We denote by

$$
\begin{gathered}
\varphi^{2}=(1-\xi) \varphi_{1}^{2}+(1-\gamma) \varphi_{2}^{2}, \quad \psi^{2}=(1-\xi) \psi_{1}^{2}+(1-\gamma) \psi_{2}^{2}, \\
\widetilde{\varphi}^{2}=(1-\xi) \widetilde{\varphi}_{1}^{2}+(1-\gamma) \widetilde{\varphi}_{2}^{2}, \quad \widetilde{\psi}^{2}=(1-\xi) \widetilde{\psi}_{1}^{2}+(1-\gamma) \widetilde{\psi}_{2}^{2}, \quad h^{2}=(1-\xi) h_{1}^{2}+(1-\gamma) h_{2}^{2} .
\end{gathered}
$$

Then we have

$$
\begin{aligned}
& (1-\xi)\left(u_{\mathrm{int}, 1}-u_{\mathrm{ext}, 1}\right)(x)+(1-\gamma)\left(u_{\mathrm{int}, 2}-u_{\mathrm{ext}, 2}\right)(x) \\
= & -4(2-\gamma-\xi) \ln \tau_{2}+8(2-\gamma-\xi) \lambda_{2} \ln \left|x-\widetilde{x}^{2}\right| \\
& +h^{2}\left(R_{\varepsilon}^{2} \frac{x-\widetilde{x}^{2}}{r_{\varepsilon}}\right)+H^{\mathrm{int}}\left(\varphi^{2}, \psi^{2} ; \frac{x-\widetilde{x}^{2}}{r_{\varepsilon}}\right)-H^{\operatorname{ext}}\left(\widetilde{\varphi}^{2}, \widetilde{\psi}^{2} ; \frac{x-\widetilde{x}^{2}}{r_{\varepsilon}}\right) \\
& -\left[(2-\gamma-\xi) H\left(x, \widetilde{x}^{2}\right)+\frac{1-\xi}{\gamma} G\left(x, \widetilde{x}^{1}\right)+\frac{1-\gamma}{\xi} G\left(x, \widetilde{x}^{3}\right)\right]+\mathcal{O}\left(\frac{\varepsilon^{2} \tau_{2}^{-2}}{\left|x-\widetilde{x}^{2}\right|^{2}}\right)+\mathcal{O}\left(r_{\varepsilon}^{2}\right) .
\end{aligned}
$$

Next, even though all functions are defined on $\partial B_{r_{\varepsilon}}\left(\widetilde{x}^{2}\right)$ in (3.34), it will be more convenient to solve on $S^{3}$, the following set of equations

$$
\begin{array}{r}
\left((1-\xi)\left(u_{\mathrm{int}, 1}-u_{\mathrm{ext}, 1}\right)+(1-\gamma)\left(u_{\mathrm{int}, 2}-u_{\mathrm{ext}, 2}\right)\right)\left(\widetilde{x}^{2}+r_{\varepsilon} \cdot\right)=0, \\
\partial_{r}\left((1-\xi)\left(u_{\mathrm{int}, 1}-u_{\mathrm{ext}, 1}\right)+(1-\gamma)\left(u_{\mathrm{int}, 2}-u_{\mathrm{ext}, 2}\right)\right)\left(\widetilde{x}^{2}+r_{\varepsilon} \cdot\right)=0, \\
\Delta\left((1-\xi)\left(u_{\mathrm{int}, 1}-u_{\mathrm{ext}, 1}\right)+(1-\gamma)\left(u_{\mathrm{int}, 2}-u_{\mathrm{ext}, 2}\right)\right)\left(\widetilde{x}^{2}+r_{\varepsilon} \cdot\right)=0, \\
\partial_{r} \Delta\left((1-\xi)\left(u_{\mathrm{int}, 1}-u_{\mathrm{ext}, 1}\right)+(1-\gamma)\left(u_{\mathrm{int}, 2}-u_{\mathrm{ext}, 2}\right)\right)\left(\widetilde{x}^{2}+r_{\varepsilon} \cdot\right)=0 .
\end{array}
$$

Since the boundary data are chosen to satisfy 3.5 or 3.6 . We decompose

$$
\begin{array}{lll}
\varphi^{2}=\varphi_{0}^{2}+\varphi_{1}^{2}+\varphi^{2, \perp}, & \psi^{2}=8 \varphi_{0}^{2}+12 \varphi_{1}^{2}+\psi^{2, \perp}, \\
\widetilde{\varphi}^{2}=\widetilde{\varphi}_{0}^{2}+\widetilde{\varphi}_{1}^{2}+\widetilde{\varphi}^{2, \perp}, & \widetilde{\psi}^{2}=\widetilde{\psi}_{1}^{2}+\widetilde{\psi}^{2, \perp},
\end{array}
$$


where $\varphi_{0}^{2}, \widetilde{\varphi}_{0}^{2} \in \mathbb{E}_{0}=\mathbb{R}$ are constant on $S^{3}, \varphi_{1}^{2}, \widetilde{\varphi}_{1}^{2}, \widetilde{\psi}_{1}^{2}$ belong to $\mathbb{E}_{1}=\operatorname{Span}\left\{e_{1}, e_{2}, e_{3}, e_{4}\right\}$ and $\varphi^{2, \perp}, \widetilde{\varphi}^{2, \perp}, \psi^{2, \perp}, \widetilde{\psi}^{2, \perp}$ are $L^{2}\left(S^{3}\right)$ orthogonal to $\mathbb{E}_{0}$ and $\mathbb{E}_{1}$.

We insist that, for $x \in S^{3}$, both equations (3.41) involve the same relation of the parameter $\tau_{2}$ and the appropriate energy $\mathcal{E}_{2}$. Then we have

$$
\begin{aligned}
& (1-\xi)\left(u_{\mathrm{int}, 1}-u_{\mathrm{ext}, 1}\right)(x)+(1-\gamma)\left(u_{\mathrm{int}, 2}-u_{\mathrm{ext}, 2}\right)\left(\widetilde{x}^{2}+r_{\varepsilon} x\right) \\
= & -4(2-\gamma-\xi) \ln \tau_{2}+8(2-\gamma-\xi) \lambda_{2} \ln r_{\varepsilon}|x|+H^{\mathrm{int}}\left(\varphi^{2}, \psi^{2} ; x\right)-H^{\mathrm{ext}}\left(\widetilde{\varphi}^{2}, \widetilde{\psi}^{2} ; x\right) \\
& -\left[(2-\gamma-\xi) H\left(\widetilde{x}^{2}, \widetilde{x}^{2}\right)+\frac{1-\xi}{\gamma} G\left(\widetilde{x}^{2}, \widetilde{x}^{1}\right)+\frac{1-\gamma}{\xi} G\left(\widetilde{x}^{2}, \widetilde{x}^{3}\right)\right]+\mathcal{O}\left(r_{\varepsilon}^{2}\right) .
\end{aligned}
$$

Projecting the set of equations 3.42 over $\mathbb{E}_{0}$, we get

$$
\begin{aligned}
-4(2-\gamma-\xi) \ln \tau_{2}+8(2-\gamma-\xi) \lambda_{2} \ln r_{\varepsilon}+\varphi_{0}^{2}-\widetilde{\varphi}_{0}^{2}-\mathcal{E}_{2}\left(\widetilde{x}^{2}, \widetilde{x}\right)+\mathcal{O}\left(r_{\varepsilon}^{2}\right) & =0 \\
8(2-\gamma-\xi) \lambda_{2}+2 \varphi_{0}^{2}+2 \widetilde{\varphi}_{0}^{2}+\mathcal{O}\left(r_{\varepsilon}^{2}\right) & =0 \\
16(2-\gamma-\xi) \lambda_{2}+8 \varphi_{0}^{2}+\mathcal{O}\left(r_{\varepsilon}^{2}\right) & =0 \\
-32(2-\gamma-\xi) \lambda_{2}+\mathcal{O}\left(r_{\varepsilon}^{2}\right) & =0
\end{aligned}
$$

where

$$
\mathcal{E}_{2}(\cdot, \widetilde{\boldsymbol{x}}):=(2-\gamma-\xi) H\left(\cdot, \widetilde{x}^{2}\right)+\frac{1-\xi}{\gamma} G\left(\cdot, \widetilde{x}^{1}\right)+\frac{1-\gamma}{\xi} G\left(\cdot, \widetilde{x}^{3}\right) .
$$

The system 3.43 can be simply written as

$$
\lambda_{2}=\mathcal{O}\left(r_{\varepsilon}^{2}\right), \quad \varphi_{0}^{2}=\mathcal{O}\left(r_{\varepsilon}^{2}\right), \quad \widetilde{\varphi}_{0}^{2}=\mathcal{O}\left(r_{\varepsilon}^{2}\right) \quad \text { and } \quad \frac{1}{\ln r_{\varepsilon}}\left[4 \ln \tau_{2}+\frac{\mathcal{E}_{2}\left(\widetilde{x}^{2}, \widetilde{\boldsymbol{x}}\right)}{2-\gamma-\xi}\right]=\mathcal{O}\left(r_{\varepsilon}^{2}\right)
$$

We are now in a position to define $\tau_{2}^{-}$and $\tau_{2}^{+}$. In fact, according to the above analysis, as $\varepsilon$ tends to 0 , we expect that $\widetilde{x}^{i}$ will converge to $x^{i}$ for $i \in\{1,2,3\}$ and $\tau_{2}$ will converge to $\tau_{2}^{*}$ satisfying

$$
4 \ln \tau_{2}^{*}=-\frac{\mathcal{E}_{2}\left(x^{2}, \boldsymbol{x}\right)}{2-\gamma-\xi}
$$

Hence it is enough to choose $\tau_{2}^{-}$and $\tau_{2}^{+}$in such a way that

$$
4 \ln \left(\tau_{2}^{-}\right)<-\frac{\mathcal{E}_{2}\left(x^{2}, \boldsymbol{x}\right)}{2-\gamma-\xi}<4 \ln \left(\tau_{2}^{+}\right)
$$

Consider now the projection of $(3.42)$ over $\mathbb{E}_{1}$. Given a smooth function $f$ defined in $\Omega$, we identify its gradient $\nabla f=\left(\partial_{x_{1}} f, \ldots, \partial_{x_{4}} f\right)$ with the element of $\mathbb{E}_{1}$,

$$
\bar{\nabla} f=\sum_{i=1}^{4} \partial_{x_{i}} f e_{i}
$$


Keeping these notations in mind, we obtain the system of equations

$$
\begin{array}{r}
\varphi_{1}^{2}-\widetilde{\varphi}_{1}^{2}-\bar{\nabla} \mathcal{E}_{2}\left(\widetilde{x}^{2}, \widetilde{\boldsymbol{x}}\right)+\mathcal{O}\left(r_{\varepsilon}^{2}\right)=0 \\
3 \varphi_{1}^{2}+3 \widetilde{\varphi}_{1}^{2}+\frac{1}{2} \widetilde{\psi}_{1}^{2}-\bar{\nabla} \mathcal{E}_{2}\left(\widetilde{x}^{2}, \widetilde{\boldsymbol{x}}\right)+\mathcal{O}\left(r_{\varepsilon}^{2}\right)=0 \\
15 \varphi_{1}^{2}-3 \widetilde{\varphi}_{1}^{2}-\widetilde{\psi}_{1}^{2}+\mathcal{O}\left(r_{\varepsilon}^{2}\right)=0 \\
15 \varphi_{1}^{2}+15 \widetilde{\varphi}_{1}^{2}+\frac{18}{4} \widetilde{\psi}_{1}^{2}+\mathcal{O}\left(r_{\varepsilon}^{2}\right)=0
\end{array}
$$

which can be simplified as follows

$$
\varphi_{1}^{2}=\mathcal{O}\left(r_{\varepsilon}^{2}\right), \quad \widetilde{\varphi}_{1}^{2}=\mathcal{O}\left(r_{\varepsilon}^{2}\right), \quad \widetilde{\psi}_{1}^{2}=\mathcal{O}\left(r_{\varepsilon}^{2}\right) \quad \text { and } \quad \bar{\nabla} \mathcal{E}_{2}\left(\widetilde{x}^{2}, \widetilde{\boldsymbol{x}}\right)=\mathcal{O}\left(r_{\varepsilon}^{2}\right)
$$

Finally, we consider the projection onto $L^{2}\left(S^{3}\right)^{\perp}$. This yields the system

$$
\begin{aligned}
& \varphi^{2, \perp}-\widetilde{\varphi}^{2, \perp}+\mathcal{O}\left(r_{\varepsilon}^{2}\right)=0, \partial_{r}\left(H_{\varphi^{2, \perp}, \psi^{2, \perp}}^{\mathrm{int}}-H_{\widetilde{\varphi}^{2, \perp}, \widetilde{\psi}^{2, \perp}}^{\mathrm{ext}}\right)+\mathcal{O}\left(r_{\varepsilon}^{2}\right)=0 \\
& \psi^{2, \perp}-\widetilde{\psi}^{2, \perp}+\mathcal{O}\left(r_{\varepsilon}^{2}\right)=0, \quad \partial_{r} \Delta\left(H_{\varphi^{2, \perp}, \psi^{2, \perp}}^{\mathrm{int}}-H_{\widetilde{\varphi}^{2, \perp}, \widetilde{\psi}^{2, \perp}}^{\mathrm{ext}}\right)+\mathcal{O}\left(r_{\varepsilon}^{2}\right)=0 .
\end{aligned}
$$

Thanks to the result of Lemma 3.8, this last system can be rewritten as

$$
\varphi^{2, \perp}=\mathcal{O}\left(r_{\varepsilon}^{2}\right), \quad \widetilde{\varphi}^{2, \perp}=\mathcal{O}\left(r_{\varepsilon}^{2}\right), \quad \psi^{2, \perp}=\mathcal{O}\left(r_{\varepsilon}^{2}\right) \quad \text { and } \quad \widetilde{\psi}^{2, \perp}=\mathcal{O}\left(r_{\varepsilon}^{2}\right)
$$

If we define the parameter $t_{2} \in \mathbb{R}$ by

$$
t_{2}=\frac{1}{\ln r_{\varepsilon}}\left[4 \ln \tau_{2}+\frac{\mathcal{E}_{2}\left(\widetilde{x}^{2}, \widetilde{\boldsymbol{x}}\right)}{2-\gamma-\xi}\right],
$$

then the systems found by projecting 3.42 gather in this equality

$$
T_{c, \varepsilon}^{2}=\left(t_{2}, \lambda_{2}, \varphi_{0}^{2}, \widetilde{\varphi}_{0}^{2}, \varphi_{1}^{2}, \widetilde{\varphi}_{1}^{2}, \widetilde{\psi}_{1}^{2}, \bar{\nabla} \mathcal{E}_{2}\left(\widetilde{x}^{2}, \widetilde{\boldsymbol{x}}\right), \varphi^{2, \perp}, \widetilde{\varphi}^{2, \perp}, \psi^{2, \perp}, \widetilde{\psi}^{2, \perp}\right)=\mathcal{O}\left(r_{\varepsilon}^{2}\right)
$$

As usual, the terms $\mathcal{O}\left(r_{\varepsilon}^{2}\right)$ depend nonlinearly on all the variables on the left side, but is bounded (in the appropriate norm) by a constant (independent of $\varepsilon$ and $\kappa$ ) times $r_{\varepsilon}^{2}$, provided $\varepsilon \in\left(0, \varepsilon_{\kappa}\right)$.

- On $\partial B_{r_{\varepsilon}}\left(\widetilde{x}^{3}\right)$, we have

$$
\begin{aligned}
\left(u_{\text {int }, 1}-u_{\text {ext }, 1}\right)(x)= & -\frac{\lambda_{1}}{\gamma} G\left(x, \widetilde{x}^{1}\right)-\lambda_{2} G\left(x, \widetilde{x}^{2}\right)+h_{1}^{3}\left(R_{\varepsilon}^{3} \frac{x-\widetilde{x}^{3}}{r_{\varepsilon}}\right) \\
& +H^{\text {int }}\left(\varphi_{1}^{3}, \psi_{1}^{3} ; \frac{x-\widetilde{x}^{3}}{r_{\varepsilon}}\right)-H^{\operatorname{ext}}\left(\widetilde{\varphi}_{1}^{3}, \widetilde{\psi}_{1}^{3} ; \frac{x-\widetilde{x}^{3}}{r_{\varepsilon}}\right)+\mathcal{O}\left(r_{\varepsilon}^{2}\right) .
\end{aligned}
$$

In the same manner as above, we will solve on $S^{3}$ the following system

$$
\begin{aligned}
\left(u_{\mathrm{int}, 1}-u_{\mathrm{ext}, 1}\right)\left(\widetilde{x}^{3}+r_{\varepsilon} \cdot\right) & =0, \quad \partial_{r}\left(u_{\mathrm{int}, 1}-u_{\mathrm{ext}, 1}\right)\left(\widetilde{x}^{3}+r_{\varepsilon} \cdot\right)=0, \\
\Delta\left(u_{\mathrm{int}, 1}-u_{\mathrm{ext}, 1}\right)\left(\widetilde{x}^{3}+r_{\varepsilon} \cdot\right) & =0, \quad \partial_{r} \Delta\left(u_{\mathrm{int}, 1}-u_{\mathrm{ext}, 1}\right)\left(\widetilde{x}^{3}+r_{\varepsilon} \cdot\right)=0 .
\end{aligned}
$$


We decompose

$$
\begin{array}{ll}
\varphi_{1}^{3}=\varphi_{1,0}^{3}+\varphi_{1,1}^{3}+\varphi_{1}^{3, \perp}, & \psi_{1}^{3}=8 \varphi_{1,0}^{3}+12 \varphi_{1,1}^{3}+\psi_{1}^{3, \perp}, \\
\widetilde{\varphi}_{1}^{3}=\widetilde{\varphi}_{1,0}^{3}+\widetilde{\varphi}_{1,1}^{3}+\widetilde{\varphi}_{1}^{3, \perp}, & \widetilde{\psi}_{1}^{3}=\widetilde{\psi}_{1,1}^{3}+\widetilde{\psi}_{1}^{3, \perp}
\end{array}
$$

with $\varphi_{1,0}^{3}, \widetilde{\varphi}_{1,0}^{3} \in \mathbb{E}_{0}, \varphi_{1,1}^{3}, \widetilde{\varphi}_{1,1}^{3}, \widetilde{\psi}_{1,1}^{3} \in \mathbb{E}_{1}$ and $\varphi_{1}^{3, \perp}, \widetilde{\varphi}_{1}^{3, \perp}, \psi_{1}^{3, \perp}, \widetilde{\psi}_{1}^{3, \perp}$ belong to $\left(L^{2}\left(S^{3}\right)\right)^{\perp}$.

Projecting the set of equations 3.45 over $\mathbb{E}_{0}$, we get

$$
\varphi_{1,0}^{3}-\widetilde{\varphi}_{1,0}^{3}+\mathcal{O}\left(r_{\varepsilon}^{2}\right)=0, \quad 2 \varphi_{1,0}^{3}+2 \widetilde{\varphi}_{1,0}^{3}+\mathcal{O}\left(r_{\varepsilon}^{2}\right)=0, \quad 8 \varphi_{1,0}^{3}+\mathcal{O}\left(r_{\varepsilon}^{2}\right)=0
$$

From the $L^{2}$-projection of 3.45 over $\mathbb{E}_{1}$, we obtain the system of equations

$$
\begin{aligned}
& \varphi_{1,1}^{3}-\widetilde{\varphi}_{1,1}^{3}+\mathcal{O}\left(r_{\varepsilon}^{2}\right)=0, 3 \varphi_{1,1}^{3}+3 \widetilde{\varphi}_{1,1}^{3}+\frac{1}{2} \widetilde{\psi}_{1,1}^{3}+\mathcal{O}\left(r_{\varepsilon}^{2}\right)=0, \\
& 15 \varphi_{1,1}^{3}-3 \widetilde{\varphi}_{1,1}^{3}-\widetilde{\psi}_{1,1}^{3}+\mathcal{O}\left(r_{\varepsilon}^{2}\right)=0, \quad 15 \varphi_{1,1}^{3}+15 \widetilde{\varphi}_{1,1}^{3}+\frac{18}{4} \widetilde{\psi}_{1,1}^{3}+\mathcal{O}\left(r_{\varepsilon}^{2}\right)=0 .
\end{aligned}
$$

Finally, we consider the $L^{2}$-projection onto $\left(L^{2}\left(S^{3}\right)\right)^{\perp}$. This yields the system

$$
\begin{aligned}
& \varphi_{1}^{3, \perp}-\widetilde{\varphi}_{1}^{3, \perp}+\mathcal{O}\left(r_{\varepsilon}^{2}\right)=0, \partial_{r}\left(H_{\varphi_{1}^{3, \perp}, \psi_{1}^{3, \perp}}^{\mathrm{int}}-H_{\widetilde{\varphi}_{1}^{3, \perp}, \widetilde{\psi}_{1}^{3, \perp}}^{\mathrm{ext}}\right)+\mathcal{O}\left(r_{\varepsilon}^{2}\right)=0 \\
& \psi_{1}^{3, \perp}-\widetilde{\psi}_{1}^{3, \perp}+\mathcal{O}\left(r_{\varepsilon}^{2}\right)=0, \quad \partial_{r} \Delta\left(H_{\varphi_{1}^{3, \perp}, \psi_{1}^{3, \perp}}^{\mathrm{int}}-H_{\widetilde{\varphi}_{1}^{3, \perp}, \widetilde{\psi}_{1}^{3, \perp}}^{\mathrm{ext}}\right)+\mathcal{O}\left(r_{\varepsilon}^{2}\right)=0 .
\end{aligned}
$$

Using again Lemma 3.8, the above system can be rewritten as

$$
\varphi_{1}^{3, \perp}=\mathcal{O}\left(r_{\varepsilon}^{2}\right), \quad \widetilde{\varphi}_{1}^{3, \perp}=\mathcal{O}\left(r_{\varepsilon}^{2}\right), \quad \psi_{1}^{3, \perp}=\mathcal{O}\left(r_{\varepsilon}^{2}\right) \quad \text { and } \quad \widetilde{\psi}_{1}^{3, \perp}=\mathcal{O}\left(r_{\varepsilon}^{2}\right) .
$$

Then the systems found by projecting (3.45) gather in this equality

$$
T_{1, \varepsilon}^{3}=\left(\varphi_{1,0}^{3}, \widetilde{\varphi}_{1,0}^{3}, \varphi_{1,1}^{3}, \widetilde{\varphi}_{1,1}^{3}, \widetilde{\psi}_{1,1}^{3}, \varphi_{1}^{3, \perp}, \widetilde{\varphi}_{1}^{3, \perp}, \psi_{1}^{3, \perp}, \widetilde{\psi}_{1}^{3, \perp}\right)=\mathcal{O}\left(r_{\varepsilon}^{2}\right)
$$

- On $\partial B_{r_{\varepsilon}}\left(\widetilde{x}^{3}\right)$, we have

$$
\begin{aligned}
& \left(u_{\mathrm{int}, 2}-u_{\mathrm{ext}, 2}\right)(x) \\
= & -\frac{4}{\xi} \ln \tau_{3}+\frac{8 \lambda_{3}}{\xi} \ln \left|x-\widetilde{x}^{3}\right|-\frac{1-\xi}{\gamma \xi} G\left(x, \widetilde{x}^{1}\right)-\frac{\ln \xi}{\xi} \\
& +h_{2}^{3}\left(R_{\varepsilon}^{3} \frac{x-\widetilde{x}^{3}}{r_{\varepsilon}}\right)+H^{\mathrm{int}}\left(\varphi_{2}^{3}, \psi_{2}^{3} ; \frac{x-\widetilde{x}^{3}}{r_{\varepsilon}}\right)-H^{\operatorname{ext}}\left(\widetilde{\varphi}_{2}^{3}, \widetilde{\psi}_{2}^{3} ; \frac{x-\widetilde{x}^{3}}{r_{\varepsilon}}\right) \\
& -\frac{1+\lambda_{3}}{\xi} H\left(x, \widetilde{x}^{3}\right)-\left(1+\lambda_{2}-\frac{1-\xi}{\xi}\right) G\left(x, \widetilde{x}^{2}\right)+\mathcal{O}\left(\frac{\varepsilon^{2} \tau_{3}^{-2}}{\left|x-\widetilde{x}^{3}\right|^{2}}\right)+\mathcal{O}\left(r_{\varepsilon}^{2}\right) .
\end{aligned}
$$

Next, even though all functions are defined on $\partial B_{r_{\varepsilon}}\left(\widetilde{x}^{3}\right)$ in 3.34 , it will be more convenient to solve on $S^{3}$ the following set of equations

$$
\begin{aligned}
& \left(u_{\text {int }, 2}-u_{\text {ext }, 2}\right)\left(\widetilde{x}^{3}+r_{\varepsilon} \cdot\right)=0, \quad \partial_{r}\left(u_{\text {int }, 2}-u_{\text {ext }, 2}\right)\left(\widetilde{x}^{3}+r_{\varepsilon} \cdot\right)=0, \\
& \Delta\left(u_{\mathrm{int}, 2}-u_{\mathrm{ext}, 2}\right)\left(\widetilde{x}^{3}+r_{\varepsilon} \cdot\right)=0, \quad \partial_{r} \Delta\left(u_{\mathrm{int}, 2}-u_{\mathrm{ext}, 2}\right)\left(\widetilde{x}^{3}+r_{\varepsilon} \cdot\right)=0 .
\end{aligned}
$$


Since the boundary data are chosen to satisfy 3.5 or 3.6 . We decompose

$$
\begin{array}{ll}
\varphi_{2}^{3}=\varphi_{2,0}^{3}+\varphi_{2,1}^{3}+\varphi_{2}^{3, \perp}, & \psi_{2}^{3}=8 \varphi_{2,0}^{3}+12 \varphi_{2,1}^{3}+\psi_{2}^{3, \perp}, \\
\widetilde{\varphi}_{2}^{3}=\widetilde{\varphi}_{2,0}^{3}+\widetilde{\varphi}_{2,1}^{3}+\widetilde{\varphi}_{2}^{3, \perp}, & \widetilde{\psi}_{2}^{3}=\widetilde{\psi}_{2,1}^{3}+\widetilde{\psi}_{2}^{3, \perp},
\end{array}
$$

where $\varphi_{2,0}^{3}, \widetilde{\varphi}_{2,0}^{3} \in \mathbb{E}_{0}=\mathbb{R}$ are constant on $S^{3}, \varphi_{2,1}^{3}, \widetilde{\varphi}_{2,1}^{3}, \widetilde{\psi}_{2,1}^{3}$ belong to $\mathbb{E}_{1}=\operatorname{Span}\left\{e_{1}, e_{2}\right.$, $\left.e_{3}, e_{4}\right\}$ and $\varphi_{2}^{3, \perp}, \widetilde{\varphi}_{2}^{3, \perp}, \psi_{2}^{3, \perp}, \widetilde{\psi}_{2}^{3, \perp}$ are $L^{2}\left(S^{3}\right)$ orthogonal to $\mathbb{E}_{0}$ and $\mathbb{E}_{1}$.

Using (3.47), we have for $x \in S^{3}$

$$
\begin{aligned}
& \left(u_{\text {int }, 2}-u_{\text {ext }, 2}\right)\left(\widetilde{x}^{3}+r_{\varepsilon} x\right) \\
= & -\frac{4}{\xi} \ln \tau_{3}+\frac{8 \lambda_{3}}{\xi} \ln \left(r_{\varepsilon}|x|\right)-\frac{1}{\xi}\left(H\left(\widetilde{x}^{3}, \widetilde{x}^{3}\right)+G\left(\widetilde{x}^{3}, \widetilde{x}^{2}\right)+\frac{1-\xi}{\gamma} G\left(\widetilde{x}^{3}, \widetilde{x}^{1}\right)\right) \\
& +H^{\text {int }}\left(\varphi_{2}^{3}, \psi_{2}^{3} ; x\right)-H^{\operatorname{ext}}\left(\widetilde{\varphi}_{2}^{3}, \widetilde{\psi}_{2}^{3} ; x\right)-\frac{\ln \xi}{\xi} \\
& -\frac{\lambda_{3}}{\xi} H\left(\widetilde{x}^{3}, \widetilde{x}^{3}\right)-\lambda_{2} G\left(\widetilde{x}^{3}, \widetilde{x}^{2}\right)+\mathcal{O}\left(r_{\varepsilon}^{2}\right) .
\end{aligned}
$$

Then, the projection of the set equations $(3.48)$ over $\mathbb{E}_{0}$ will yield

$$
\begin{aligned}
-4 \ln \tau_{3}+8 \lambda_{3} \ln r_{\varepsilon}-\ln \xi+\xi \varphi_{2,0}^{3}-\xi \widetilde{\varphi}_{2,0}^{3}-\mathcal{E}_{3}\left(\widetilde{x}^{3}, \widetilde{\boldsymbol{x}}\right)+\mathcal{O}\left(r_{\varepsilon}^{2}\right) & =0, \\
8 \lambda_{3}+2 \xi \varphi_{2,0}^{3}+2 \xi \widetilde{\varphi}_{2,0}^{3}+\mathcal{O}\left(r_{\varepsilon}^{2}\right) & =0, \\
16 \lambda_{3}+8 \xi \varphi_{2,0}^{3}+\mathcal{O}\left(r_{\varepsilon}^{2}\right) & =0, \\
-32 \lambda_{3}+\mathcal{O}\left(r_{\varepsilon}^{2}\right) & =0,
\end{aligned}
$$

where

$$
\mathcal{E}_{3}(\cdot, \widetilde{\boldsymbol{x}}):=H\left(\cdot, \widetilde{x}^{3}\right)+G\left(\cdot, \widetilde{x}^{2}\right)+\frac{1-\xi}{\gamma} G\left(\cdot, \widetilde{x}^{1}\right) .
$$

The system 3.49 can be simply written as

$\lambda_{3}=\mathcal{O}\left(r_{\varepsilon}^{2}\right), \quad \varphi_{2,0}^{3}=\mathcal{O}\left(r_{\varepsilon}^{2}\right), \quad \widetilde{\varphi}_{2,0}^{3}=\mathcal{O}\left(r_{\varepsilon}^{2}\right) \quad$ and $\quad \frac{1}{\ln r_{\varepsilon}}\left[4 \ln \tau_{3}+\ln \xi+\mathcal{E}_{3}\left(\widetilde{x}^{3}, \widetilde{\boldsymbol{x}}\right)\right]=\mathcal{O}\left(r_{\varepsilon}^{2}\right)$.

We are now in a position to define $\tau_{3}^{-}$and $\tau_{3}^{+}$. In fact, according to the above analysis, as $\varepsilon$ tends to 0 , we expect that $\widetilde{x}^{i}$ will converge to $x^{i}$ for $i \in\{1,2,3\}$ and $\tau_{3}$ will converge to $\tau_{3}^{*}$ satisfying

$$
4 \ln \tau_{3}^{*}=-\ln \xi-\mathcal{E}_{3}\left(x^{3}, \boldsymbol{x}\right) .
$$

Hence it is enough to choose $\tau_{3}^{-}$and $\tau_{3}^{+}$in such a way that

$$
4 \ln \left(\tau_{3}^{-}\right)<-\ln \xi-\mathcal{E}_{3}\left(x^{3}, \boldsymbol{x}\right)<4 \ln \left(\tau_{3}^{+}\right) .
$$

Consider now the projection of $(3.48)$ over $\mathbb{E}_{1}$. Given a smooth function $f$ defined in $\Omega$, we identify its gradient $\nabla f=\left(\partial_{x_{1}} f, \ldots, \partial_{x_{4}} f\right)$ with the element of $\mathbb{E}_{1}$,

$$
\bar{\nabla} f=\sum_{i=1}^{4} \partial_{x_{i}} f e_{i} .
$$


Keeping these notations in mind, we obtain the system of equations

$$
\begin{aligned}
\varphi_{2,1}^{3}-\widetilde{\varphi}_{2,1}^{3}-\bar{\nabla} \mathcal{E}_{3}\left(\widetilde{x}^{3}, \widetilde{\boldsymbol{x}}\right)+\mathcal{O}\left(r_{\varepsilon}^{2}\right) & =0, \\
3 \varphi_{2,1}^{3}+3 \widetilde{\varphi}_{2,1}^{3}+\frac{1}{2} \widetilde{\psi}_{2,1}^{3}-\bar{\nabla} \mathcal{E}_{3}\left(\widetilde{x}^{3}, \widetilde{\boldsymbol{x}}\right)+\mathcal{O}\left(r_{\varepsilon}^{2}\right) & =0 \\
15 \varphi_{2,1}^{3}-3 \widetilde{\varphi}_{2,1}^{3}-\widetilde{\psi}_{2,1}^{3}+\mathcal{O}\left(r_{\varepsilon}^{2}\right) & =0, \\
15 \varphi_{2,1}^{3}+15 \widetilde{\varphi}_{2,1}^{3}+\frac{18}{4} \widetilde{\psi}_{2,1}^{3}+\mathcal{O}\left(r_{\varepsilon}^{2}\right) & =0,
\end{aligned}
$$

which can be simplified as follows

$$
\varphi_{2,1}^{3}=\mathcal{O}\left(r_{\varepsilon}^{2}\right), \quad \widetilde{\varphi}_{2,1}^{3}=\mathcal{O}\left(r_{\varepsilon}^{2}\right), \quad \widetilde{\psi}_{2,1}^{3}=\mathcal{O}\left(r_{\varepsilon}^{2}\right) \quad \text { and } \quad \bar{\nabla} \mathcal{E}_{3}\left(\widetilde{x}^{3}, \widetilde{\boldsymbol{x}}\right)=\mathcal{O}\left(r_{\varepsilon}^{2}\right) .
$$

Finally, we consider the projection onto $L^{2}\left(S^{3}\right)^{\perp}$. This yields the system

$$
\begin{aligned}
& \varphi_{2}^{3, \perp}-\widetilde{\varphi}_{2}^{3, \perp}+\mathcal{O}\left(r_{\varepsilon}^{2}\right)=0, \quad \partial_{r}\left(H_{\varphi_{2}^{3, \perp}, \psi_{2}^{3, \perp}}^{\mathrm{int}}-H_{\widetilde{\varphi}_{2}^{3, \perp}, \widetilde{\psi}_{2}^{3, \perp}}^{\mathrm{ext}}\right)+\mathcal{O}\left(r_{\varepsilon}^{2}\right)=0 \\
& \psi_{2}^{3, \perp}-\widetilde{\psi}_{2}^{3, \perp}+\mathcal{O}\left(r_{\varepsilon}^{2}\right)=0, \quad \partial_{r} \Delta\left(H_{\varphi_{2}^{3, \perp}, \psi_{2}^{3, \perp}}^{\mathrm{int}}-H_{\widetilde{\varphi}_{2}^{3, \perp}, \widetilde{\psi}_{2}^{3, \perp}}^{\mathrm{ext}}\right)+\mathcal{O}\left(r_{\varepsilon}^{2}\right)=0 .
\end{aligned}
$$

Thanks to the result of Lemma 3.8, this last system can be rewritten as

$$
\varphi_{2}^{3, \perp}=\mathcal{O}\left(r_{\varepsilon}^{2}\right), \quad \widetilde{\varphi}_{2}^{3, \perp}=\mathcal{O}\left(r_{\varepsilon}^{2}\right), \quad \psi_{2}^{3, \perp}=\mathcal{O}\left(r_{\varepsilon}^{2}\right) \quad \text { and } \quad \widetilde{\psi}_{2}^{3, \perp}=\mathcal{O}\left(r_{\varepsilon}^{2}\right) .
$$

If we define the parameter $t_{3} \in \mathbb{R}$ by

$$
t_{3}=\frac{1}{\ln r_{\varepsilon}}\left[4 \ln \tau_{3}+\ln \xi+\mathcal{E}_{3}\left(\widetilde{x}^{3}, \widetilde{\boldsymbol{x}}\right)\right],
$$

then the systems found by projecting 3.48 gather in this equality

$$
T_{2, \varepsilon}^{3}=\left(t_{3}, \lambda_{3}, \varphi_{2,0}^{3}, \widetilde{\varphi}_{2,0}^{3}, \varphi_{2,1}^{3}, \widetilde{\varphi}_{2,1}^{3}, \widetilde{\psi}_{2,1}^{3} \bar{\nabla} \mathcal{E}_{3}\left(\widetilde{x}^{3}, \widetilde{\boldsymbol{x}}\right), \varphi_{2}^{3, \perp}, \widetilde{\varphi}_{2}^{3, \perp}, \psi_{2}^{3, \perp}, \widetilde{\psi}_{2}^{3, \perp}\right)=\mathcal{O}\left(r_{\varepsilon}^{2}\right)
$$

As usual, the terms $\mathcal{O}\left(r_{\varepsilon}^{2}\right)$ depend nonlinearly on all the variables on the left side, but is bounded (in the appropriate norm) by a constant (independent of $\varepsilon$ and $\kappa$ ) times $r_{\varepsilon}^{2}$, provided $\varepsilon \in\left(0, \varepsilon_{\kappa}\right)$.

We recall that $\boldsymbol{d}=r_{\varepsilon}(\widetilde{\boldsymbol{x}}-\boldsymbol{x})$, in addition the previous systems can be written as for $i=1,2,3$ :

$$
\left(\boldsymbol{d}, t_{i}, \lambda_{i}, \varphi^{i}, \widetilde{\varphi}^{i}, \psi^{i}, \widetilde{\psi}^{i}, \bar{\nabla} \mathcal{E}_{i}\right)=\mathcal{O}\left(r_{\varepsilon}^{2}\right) .
$$

Combining (3.38), 3.40), 3.44), 3.46) and (3.50), we have

$$
T_{i, \varepsilon}=\left(T_{i, \varepsilon}^{1}, T_{i, \varepsilon}^{2}, T_{i, \varepsilon}^{3}\right)=\left(\mathcal{O}\left(r_{\varepsilon}^{2}\right), \mathcal{O}\left(r_{\varepsilon}^{2}\right), \mathcal{O}\left(r_{\varepsilon}^{2}\right)\right) \quad \text { for } i=1,2 .
$$

Then the nonlinear mapping which appears on the right-hand side of (3.51) is continuous, compact. In addition, reducing $\varepsilon_{\kappa}$ if necessary, this nonlinear mapping sends the ball of radius $\kappa r_{\varepsilon}^{2}$ (for the natural product norm) into itself, provided $\kappa$ is fixed large enough. Applying Schauder's fixed point Theorem in the ball of radius $\kappa r_{\varepsilon}^{2}$ in the product space where the entries live, we obtain the existence of a solution of equation (3.51). This completes the proof of Theorem 1.5 . 


\section{Proof of Theorem 1.6}

\subsection{Construction of the approximate solution}

\subsubsection{Ansatz and first estimates}

We define another ansatz for solution of 1.1 :

$$
\widetilde{u}_{1}(x)= \begin{cases}\frac{1}{\gamma} u_{\varepsilon, \tau_{1}}\left(x-x^{1}\right)-\frac{1-\gamma}{\gamma} G\left(x, x^{2}\right)-\frac{1-\gamma}{\gamma \xi} G\left(x, x^{3}\right)-\frac{\ln \gamma}{\gamma}, & x \in B_{r_{\varepsilon}}\left(x^{1}\right), \\ u_{\varepsilon, \tau_{2}}\left(x-x^{2}\right)+\frac{1-\gamma}{\gamma(2-\gamma-\xi)} G\left(x, x^{1}\right)-\frac{1-\gamma}{\xi(2-\gamma-\xi)} G\left(x, x^{3}\right), & x \in B_{r_{\varepsilon}}\left(x^{2}\right), \\ \frac{1}{\gamma} G\left(x, x^{1}\right)+G\left(x, x^{2}\right), & x \in \Omega \backslash \bigcup_{i=1}^{2} B_{r_{\varepsilon}}\left(x^{i}\right)\end{cases}
$$

and

$$
\widetilde{u}_{2}(x)= \begin{cases}\frac{1}{\xi} u_{\varepsilon, \tau_{3}}\left(x-x^{3}\right)-\frac{1-\xi}{\xi} G\left(x, x^{2}\right)-\frac{1-\xi}{\gamma \xi} G\left(x, x^{1}\right)-\frac{\ln \xi}{\xi}, & x \in B_{r_{\varepsilon}}\left(x^{3}\right), \\ u_{\varepsilon, \tau_{2}}\left(x-x^{2}\right)-\frac{1-\xi}{\gamma(2-\gamma-\xi)} G\left(x, x^{1}\right)+\frac{1-\xi}{\xi(2-\gamma-\xi)} G\left(x, x^{3}\right), & x \in B_{r_{\varepsilon}}\left(x^{2}\right), \\ \frac{1}{\xi} G\left(x, x^{3}\right)+G\left(x, x^{2}\right), & x \in \Omega \backslash \bigcup_{i=2}^{3} B_{r_{\varepsilon}}\left(x^{i}\right) .\end{cases}
$$

Therefore, in $B_{r_{\varepsilon}}\left(x^{1}\right)$, there holds

$$
\begin{aligned}
& \Delta^{2} \widetilde{u}_{1}-\rho^{4} e^{\gamma \widetilde{u}_{1}+(1-\gamma) \widetilde{u}_{2}}=0 \\
& \Delta^{2} \widetilde{u}_{2}-\rho^{4} e^{\xi \widetilde{u}_{2}+(1-\xi) \widetilde{u}_{1}}=\frac{-384 \varepsilon^{4} \tau_{1}^{4 \frac{1-\xi}{\gamma}}\left(1+\varepsilon^{2}\right)^{4 \frac{1-\gamma-\xi}{\gamma}}}{\gamma^{\frac{1-\xi}{\gamma}}\left(\varepsilon^{2}+\tau_{1}^{2}\left|x-x^{1}\right|^{2}\right)^{4 \frac{1-\xi}{\gamma}}} e^{\frac{\gamma+\xi-1}{\gamma} G\left(x, x^{2}\right)+\frac{\gamma+\xi-1}{\gamma \xi} G\left(x, x^{3}\right)} .
\end{aligned}
$$

Then, for $r=\left|x-x^{1}\right|$ and $0<\delta<(\gamma+\xi-1) / \gamma$, we have

$$
\begin{aligned}
\left\|\Delta^{2} \widetilde{u}_{2}-\rho^{4} e^{\xi \widetilde{u}_{2}+(1-\xi) \widetilde{u}_{1}}\right\|_{\mathcal{C}_{\delta-4}^{0, \alpha}\left(B_{r_{\varepsilon}}\left(x^{1}\right)\right)} & \leq C \sup _{r<r_{\varepsilon}} \frac{\tau_{1}^{4 \frac{1-\xi}{\gamma}} \varepsilon^{4}}{\gamma^{\frac{1-\xi}{\gamma}}\left(1+\varepsilon^{2}\right)^{4-4 \frac{1-\xi}{\gamma}}} \frac{r^{4-\delta}}{\varepsilon^{8 \frac{1-\xi}{\gamma}}\left(1+\left(\frac{\tau_{1}}{\varepsilon} r\right)^{2}\right)^{4 \frac{1-\xi}{\gamma}}} \\
& \leq C \sup _{r<R_{\varepsilon}^{1}} \frac{\varepsilon^{8-8 \frac{1-\xi}{\gamma}-\delta}}{\left(1+\varepsilon^{2}\right)^{4-4 \frac{1-\xi}{\gamma}}} \frac{r^{4-\delta}}{\left(1+r^{2}\right)^{4 \frac{1-\xi}{\gamma}}} \\
& \leq C \sup _{r<R_{\varepsilon}^{1}} \varepsilon^{8-8 \frac{1-\xi}{\gamma}-\delta} S(r),
\end{aligned}
$$

where $S(r)=\frac{r^{4-\delta}}{\left(1+r^{2}\right)^{4 \frac{1-\xi}{\gamma}}}$.

If $4-\delta-8(1-\xi) / \gamma \leq 0$, then $S$ is bounded on $\mathbb{R}_{+}$, hence

$$
\left\|\Delta^{2} \widetilde{u}_{2}-\rho^{4} e^{\xi \widetilde{u}_{2}+(1-\xi) \widetilde{u}_{1}}\right\|_{\mathcal{C}_{\delta-4}^{0, \alpha}\left(B_{r_{\varepsilon}}\left(x^{1}\right)\right)} \leq C \varepsilon^{8-8 \frac{1-\xi}{\gamma}-\delta} \leq C r_{\varepsilon}^{2} .
$$

If $4-\delta-8(1-\xi) / \gamma>0, \sup _{\left[0, r_{\varepsilon} / \varepsilon\right)} S(r)=S\left(r_{\varepsilon} / \varepsilon\right)$, then

$$
\left\|\Delta^{2} \widetilde{u}_{2}-\rho^{4} e^{\xi \widetilde{u}_{2}+(1-\xi) \widetilde{u}_{1}}\right\|_{\mathcal{C}_{\delta-4}^{0, \alpha}\left(B_{r_{\varepsilon}}\left(x^{1}\right)\right)} \leq C r_{\varepsilon}^{2} \quad \text { as } \varepsilon^{8-8 \frac{1-\xi}{\gamma}-\delta} S\left(\frac{r_{\varepsilon}}{\varepsilon}\right) \leq C r_{\varepsilon}^{2}
$$


On the other hand, in $B_{r_{\varepsilon}}\left(x^{2}\right)$, there holds

$$
\begin{aligned}
\Delta^{2} \widetilde{u}_{1}-\rho^{4} e^{\gamma \widetilde{u}_{1}+(1-\gamma) \widetilde{u}_{2}} & =\frac{-384 \varepsilon^{4} \tau_{2}^{4}}{\left(\varepsilon^{2}+\tau_{2}^{2}\left|x-x^{2}\right|^{2}\right)^{4}}\left(e^{\frac{(1-\gamma)(\gamma+\xi-1)}{(2-\gamma-\xi)}\left(\frac{1}{\gamma} G\left(x, x^{1}\right)-\frac{1}{\xi} G\left(x, x^{3}\right)\right)}-1\right), \\
\Delta^{2} \widetilde{u}_{2}-\rho^{4} e^{\xi \widetilde{u}_{2}+(1-\xi) \widetilde{u}_{1}} & =\frac{-384 \varepsilon^{4} \tau_{2}^{4}}{\left(\varepsilon^{2}+\tau_{2}^{2}\left|x-x^{2}\right|^{2}\right)^{4}}\left(e^{\frac{(1-\xi)(\gamma+\xi-1)}{(2-\gamma-\xi)}\left(\frac{-1}{\gamma} G\left(x, x^{1}\right)+\frac{1}{\xi} G\left(x, x^{3}\right)\right)}-1\right) .
\end{aligned}
$$

Then, for $r=\left|x-x^{2}\right|, \mu \in(1,2)$ and using the condition 1.6$)$, we have the following estimates

$$
\left\|\Delta^{2} \widetilde{u}_{1}-\rho^{4} e^{\gamma \widetilde{u}_{1}+(1-\gamma) \widetilde{u}_{2}}\right\|_{\mathcal{C}_{\mu-4}^{0, \alpha}\left(B_{r_{\varepsilon}}\left(x^{2}\right)\right)} \leq C r_{\varepsilon}^{2}
$$

and

$$
\left\|\Delta^{2} \widetilde{u}_{1}-\rho^{4} e^{\xi \widetilde{u}_{1}+(1-\xi) \widetilde{u}_{2}}\right\|_{\mathcal{C}_{\mu-4}^{0, \alpha}\left(B_{r_{\varepsilon}}\left(x^{2}\right)\right)} \leq C r_{\varepsilon}^{2}
$$

Finally, in $B_{r_{\varepsilon}}\left(x^{3}\right)$, there holds

$$
\begin{aligned}
& \Delta^{2} \widetilde{u}_{1}-\rho^{4} e^{\gamma \widetilde{u}_{1}+(1-\gamma) \widetilde{u}_{2}}=\frac{-384 \varepsilon^{4} \tau_{3}^{4 \frac{1-\gamma}{\xi}}\left(1+\varepsilon^{2}\right)^{4(1-\xi-\gamma) / \xi}}{\xi^{\frac{1-\gamma}{\xi}}\left(\varepsilon^{2}+\tau_{3}^{2}\left|x-x^{3}\right|^{2}\right)^{4 \frac{1-\gamma}{\xi}}} e^{\frac{\gamma+\xi-1}{\xi} G\left(x, x^{2}\right)+\frac{\gamma+\xi-1}{\xi \gamma} G\left(x, x^{1}\right)}, \\
& \Delta^{2} \widetilde{u}_{2}-\rho^{4} e^{\xi \widetilde{u}_{2}+(1-\xi) \widetilde{u}_{1}}=0 .
\end{aligned}
$$

Then, for $r=\left|x-x^{3}\right|$ and $0<\delta<(\gamma+\xi-1) / \xi$, we have the same estimates

$$
\left\|\Delta^{2} \widetilde{u}_{1}-\rho^{4} e^{\gamma \widetilde{u}_{1}+(1-\gamma) \widetilde{u}_{2}}\right\|_{\mathcal{C}_{\delta-4}^{0, \alpha}\left(B_{r_{\varepsilon}}\left(x^{3}\right)\right)} \leq C r_{\varepsilon}^{2}
$$

\subsection{The nonlinear interior problem}

Here, we are interested to study the system

$$
\Delta^{2} u_{1}=\rho^{4} e^{\gamma u_{1}+(1-\gamma) u_{2}}, \quad \Delta^{2} u_{2}=\rho^{4} e^{\xi u_{2}+(1-\xi) u_{1}} .
$$

Using the following transformations

$$
\begin{aligned}
& \begin{cases}v_{1}(x)=u_{1}\left(\frac{\varepsilon}{\tau_{1}} x\right)+\frac{8}{\gamma} \ln \varepsilon-\frac{4}{\gamma} \ln \left(\frac{\tau_{1}\left(1+\varepsilon^{2}\right)}{2}\right) & \text { in } B_{r_{\varepsilon}}\left(x^{1}\right), \\
v_{2}(x)=u_{2}\left(\frac{\varepsilon}{\tau_{1}} x\right) & \text { in } B_{r_{\varepsilon}}\left(x^{1}\right),\end{cases} \\
& \begin{cases}v_{1}(x)=u_{1}\left(\frac{\varepsilon}{\tau_{2}} x\right)+8 \ln \varepsilon-4 \ln \left(\frac{\tau_{2}\left(1+\varepsilon^{2}\right)}{2}\right) & \text { in } B_{r_{\varepsilon}}\left(x^{2}\right), \\
v_{2}(x)=u_{2}\left(\frac{\varepsilon}{\tau_{2}} x\right)+8 \ln \varepsilon-4 \ln \left(\frac{\tau_{2}\left(1+\varepsilon^{2}\right)}{2}\right) & \text { in } B_{r_{\varepsilon}}\left(x^{2}\right)\end{cases}
\end{aligned}
$$

and

$$
\begin{cases}v_{1}(x)=u_{1}\left(\frac{\varepsilon}{\tau_{3}} x\right) & \text { in } B_{r_{\varepsilon}}\left(x^{3}\right) \\ v_{2}(x)=u_{2}\left(\frac{\varepsilon}{\tau_{3}} x\right)+\frac{8}{\xi} \ln \varepsilon-\frac{4}{\xi} \ln \left(\frac{\tau_{3}\left(1+\varepsilon^{2}\right)}{2}\right) & \text { in } B_{r_{\varepsilon}}\left(x^{3}\right)\end{cases}
$$


So the previous systems can be written as

$$
\begin{gathered}
\begin{cases}\Delta^{2} v_{1}=24 e^{\gamma v_{1}+(1-\gamma) v_{2}} & \text { in } B_{R_{\varepsilon}^{1}}\left(x^{1}\right), \\
\Delta^{2} v_{2}=24 C_{1, \varepsilon}^{4 \frac{\gamma+\xi-1}{\gamma}} \varepsilon^{8 \frac{\gamma+\xi-1}{\gamma}} e^{\xi v_{2}+(1-\xi) v_{1}} & \text { in } B_{R_{\varepsilon}^{1}}\left(x^{1}\right),\end{cases} \\
\begin{cases}\Delta^{2} v_{1}=24 e^{\gamma v_{1}+(1-\gamma) v_{2}} & \text { in } B_{R_{\varepsilon}^{2}}\left(x^{2}\right), \\
\Delta^{2} v_{2}=24 e^{\xi v_{2}+(1-\xi) v_{1}} & \text { in } B_{R_{\varepsilon}^{2}}\left(x^{2}\right)\end{cases}
\end{gathered}
$$

and

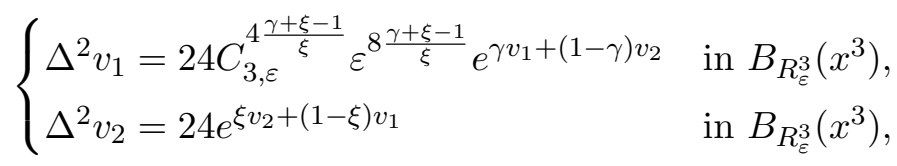

where $C_{i, \varepsilon}=\frac{2}{\tau_{i}\left(1+\varepsilon^{2}\right)}$ for $i=1,3$. Here $\tau_{i}>0$ is a constant which will be fixed later.

Given $\varphi^{i}:=\left(\varphi_{1}^{i}, \varphi_{2}^{i}\right) \in\left(\mathcal{C}^{4, \alpha}\left(S^{3}\right)\right)^{2}$ and $\psi^{i}:=\left(\psi_{1}^{i}, \psi_{2}^{i}\right) \in\left(\mathcal{C}^{2, \alpha}\left(S^{3}\right)\right)^{2}$ such that $\left(\varphi_{1}^{i}, \psi_{1}^{i}\right)$ and $\left(\varphi_{2}^{i}, \psi_{2}^{i}\right)$ satisfy $(3.5)$. We denote by $\bar{u}=u_{\varepsilon=1, \tau_{i}=1}$.

In $B_{R_{\varepsilon}^{1}}\left(x^{1}\right)$ and $B_{R_{\varepsilon}^{3}}\left(x^{3}\right)$, we reproduce exactly the same as in the proof of Theorem 1.5 . so we have the following propositions.

Proposition 4.1. Given $\kappa>0, \mu \in(1,2)$ and $\delta \in\left(0, \min \left\{\left(\frac{\gamma+\xi-1}{\gamma}\right),\left(\frac{\gamma+\xi-1}{\xi}\right)\right\}\right)$, there exist $\varepsilon_{\kappa}>0, c_{\kappa}>0$ and $\gamma_{0} \in(0,1)$ such that for all $\varepsilon \in\left(0, \varepsilon_{\kappa}\right), \gamma \in\left(\gamma_{0}, 1\right)$, for all $\tau_{1}$ in some fixed compact subset of $\left[\tau_{1}^{-}, \tau_{1}^{+}\right] \subset(0, \infty)$ and for $\varphi_{j}^{1}$ and $\psi_{j}^{1}$ satisfying (3.5) and 3.13, there exists a unique $\left(h_{1}^{1}, h_{2}^{1}\right)\left(:=\left(h_{1, \varepsilon, \tau_{1}, \varphi_{1}^{1}, \psi_{1}^{1}}, h_{2, \varepsilon, \tau_{1}, \varphi_{2}^{1}, \psi_{2}^{1}}\right)\right)$ solution of 3.12 such that

$$
\left\|\left(h_{1}^{1}, h_{2}^{1}\right)\right\|_{C_{\mu}^{4, \alpha}\left(\mathbb{R}^{4}\right) \times C_{\delta}^{4, \alpha}\left(\mathbb{R}^{4}\right)} \leq 2 c_{\kappa} r_{\varepsilon}^{2} .
$$

Hence

$$
\begin{aligned}
v_{1}(x):= & \frac{1}{\gamma} \bar{u}\left(x-x^{1}\right)-\frac{1-\gamma}{\gamma} G\left(\frac{\varepsilon x}{\tau_{1}}, x^{2}\right)-\frac{1-\gamma}{\gamma \xi} G\left(\frac{\varepsilon x}{\tau_{1}}, x^{3}\right) \\
& -\frac{\ln \gamma}{\gamma}+h_{1}^{1}(x)+H^{\operatorname{int}}\left(\varphi_{1}^{1}, \psi_{1}^{1} ; \frac{x-x^{1}}{R_{\varepsilon}^{1}}\right), \\
v_{2}(x):= & \frac{1}{\xi} G\left(\frac{\varepsilon x}{\tau_{1}}, x^{3}\right)+G\left(\frac{\varepsilon x}{\tau_{1}}, x^{2}\right)+h_{2}^{1}(x)+H^{\text {int }}\left(\varphi_{2}^{1}, \psi_{2}^{1} ; \frac{x-x^{1}}{R_{\varepsilon}^{1}}\right)
\end{aligned}
$$

solves 4.1 in $B_{R_{\varepsilon}^{1}}\left(x^{1}\right)$.

Proposition 4.2. Given $\kappa>0, \mu \in(1,2)$ and $\delta \in\left(0, \min \left\{\left(\frac{\gamma+\xi-1}{\gamma}\right),\left(\frac{\gamma+\xi-1}{\xi}\right)\right\}\right)$, there exist $\varepsilon_{\kappa}>0, c_{\kappa}>0$ and $\xi_{0} \in(0,1)$ such that for all $\varepsilon \in\left(0, \varepsilon_{\kappa}\right), \xi \in\left(\xi_{0}, 1\right)$, for all $\tau_{3}$ in some fixed compact subset of $\left[\tau_{3}^{-}, \tau_{3}^{+}\right] \subset(0, \infty)$ and for $\varphi_{j}^{3}$ and $\psi_{j}^{3}$ satisfying (3.5) and (3.17),

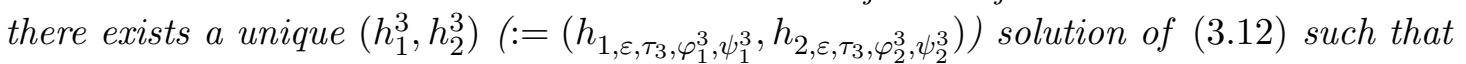

$$
\left\|\left(h_{1}^{3}, h_{2}^{3}\right)\right\|_{C_{\delta}^{4, \alpha}\left(\mathbb{R}^{4}\right) \times C_{\mu}^{4, \alpha}\left(\mathbb{R}^{4}\right)} \leq 2 c_{\kappa} r_{\varepsilon}^{2} .
$$




\section{Hence}

$$
\begin{aligned}
v_{1}(x):= & \frac{1}{\gamma} G\left(\frac{\varepsilon x}{\tau_{3}}, x^{1}\right)+G\left(\frac{\varepsilon x}{\tau_{3}}, x^{2}\right)+h_{1}^{3}(x)+H^{\text {int }}\left(\varphi_{1}^{3}, \psi_{1}^{3} ; \frac{x-x^{3}}{R_{\varepsilon}^{3}}\right), \\
v_{2}(x):= & \frac{1}{\xi} \bar{u}\left(x-x^{3}\right)-\frac{1-\xi}{\xi} G\left(\frac{\varepsilon x}{\tau_{3}}, x^{2}\right)-\frac{1-\xi}{\gamma \xi} G\left(\frac{\varepsilon x}{\tau_{3}}, x^{1}\right) \\
& -\frac{\ln \xi}{\xi}+h_{2}^{3}(x)+H^{\text {int }}\left(\varphi_{2}^{3}, \psi_{2}^{3} ; \frac{x-x^{3}}{R_{\varepsilon}^{3}}\right)
\end{aligned}
$$

solves 4.3 in $B_{R_{\varepsilon}^{3}}\left(x^{3}\right)$.

In $B_{R_{\varepsilon}^{2}}\left(x^{2}\right)$ we look for a solution of 4.2 of the form

$$
\begin{aligned}
v_{1}(x)= & \bar{u}\left(x-x^{2}\right)+\frac{1-\gamma}{\gamma(2-\gamma-\xi)} G\left(\frac{\varepsilon x}{\tau_{2}}, x^{1}\right)-\frac{1-\gamma}{\xi(2-\gamma-\xi)} G\left(\frac{\varepsilon x}{\tau_{2}}, x^{3}\right) \\
& +H^{\operatorname{int}}\left(\varphi_{1}^{2}, \psi_{1}^{2} ; \frac{x-x^{2}}{R_{\varepsilon}^{2}}\right)+h_{1}^{2}(x), \\
v_{2}(x)= & \bar{u}\left(x-x^{2}\right)-\frac{1-\xi}{\gamma(2-\gamma-\xi)} G\left(\frac{\varepsilon x}{\tau_{2}}, x^{1}\right)+\frac{1-\xi}{\xi(2-\gamma-\xi)} G\left(\frac{\varepsilon x}{\tau_{2}}, x^{3}\right) \\
& +H^{\operatorname{int}}\left(\varphi_{2}^{2}, \psi_{2}^{2} ; \frac{x-x^{2}}{R_{\varepsilon}^{2}}\right)+h_{2}^{2}(x) .
\end{aligned}
$$

This amounts to solve the equations

$$
\begin{aligned}
& \mathbb{L} h_{1}^{2}=\frac{384}{\left(1+r^{2}\right)^{4}}\left[e^{\frac{(1-\gamma)(\gamma+\xi-1)}{(2-\gamma-\xi)}\left(\frac{1}{\gamma} G\left(\frac{\varepsilon x}{\tau_{2}}, x^{1}\right)-\frac{1}{\xi} G\left(\frac{\varepsilon x}{\tau_{2}}, x^{3}\right)\right)+\gamma\left(h_{1}^{2}+H_{\varphi_{1}^{2}, \psi_{1}^{2}}^{\mathrm{int}}\right)+(1-\gamma)\left(h_{2}^{2}+H_{\varphi_{2}^{2}}^{\mathrm{int}}, \psi_{2}^{2}\right)}-h_{1}^{2}-1\right], \\
& \mathbb{L} h_{2}^{2}=\frac{384}{\left(1+r^{2}\right)^{4}}\left[e^{\frac{(1-\xi)(\gamma+\xi-1)}{(2-\gamma-\xi)}\left(-\frac{1}{\gamma} G\left(\frac{\varepsilon x}{\tau_{2}}, x^{1}\right)+\frac{1}{\xi} G\left(\frac{\varepsilon x}{\tau_{2}}, x^{3}\right)\right)+\xi\left(h_{2}^{2}+H_{\varphi_{2}^{2}}^{\mathrm{int}}, \psi_{2}^{2}\right)+(1-\xi)\left(h_{1}^{2}+H_{\varphi_{1}^{2}, \psi_{1}^{2}}^{\mathrm{int}}\right)}-h_{2}^{2}-1\right] .
\end{aligned}
$$

We denote by

$$
\mathbb{L} h_{1}^{2}=\mathcal{T}_{3}\left(h_{1}^{2}, h_{2}^{2}\right) \quad \text { and } \quad \mathbb{L} h_{2}^{2}=\mathcal{T}_{4}\left(h_{1}^{2}, h_{2}^{2}\right) .
$$

To find a solution of (4.4), it is enough to find a fixed point $\left(h_{1}^{2}, h_{2}^{2}\right)$ in a small ball of $\mathcal{C}_{\mu}^{4, \alpha}\left(\mathbb{R}^{4}\right) \times \mathcal{C}_{\mu}^{4, \alpha}\left(\mathbb{R}^{4}\right)$, solutions of

$$
\begin{aligned}
& h_{1}^{2}=\mathcal{G}_{\mu} \circ \xi_{\mu} \circ \mathcal{T}_{3}\left(h_{1}^{2}, h_{2}^{2}\right)=\mathcal{N}_{2}\left(h_{1}^{2}, h_{2}^{2}\right), \\
& h_{2}^{2}=\mathcal{G}_{\mu} \circ \xi_{\mu} \circ \mathcal{T}_{4}\left(h_{1}^{2}, h_{2}^{2}\right)=\mathcal{M}_{2}\left(h_{1}^{2}, h_{2}^{2}\right) .
\end{aligned}
$$

Then, we have the following result.

Lemma 4.3. Let $\mu \in(1,2), \gamma_{0}$ and $\xi_{0} \in(0,1)$. Given $\kappa>0$, there exist $\varepsilon_{\kappa}>0$ and $c_{\kappa}>0$ such that for all $\varepsilon \in\left(0, \varepsilon_{\kappa}\right), \gamma \in\left(\gamma_{0}, 1\right)$ and $\xi \in\left(\xi_{0}, 1\right)$. We have

$$
\begin{gathered}
\left\|\mathcal{N}_{2}(0,0)\right\|_{\mathcal{C}_{\mu}^{4, \alpha}\left(\mathbb{R}^{4}\right)} \leq c_{\kappa} r_{\varepsilon}^{2}, \quad\left\|\mathcal{M}_{2}(0,0)\right\|_{\mathcal{C}_{\mu}^{4, \alpha}\left(\mathbb{R}^{4}\right)} \leq c_{\kappa} r_{\varepsilon}^{2} \\
\left\|\mathcal{N}_{2}\left(h_{1}^{2}, h_{2}^{2}\right)-\mathcal{N}_{2}\left(k_{1}^{2}, k_{2}^{2}\right)\right\|_{\mathcal{C}_{\mu}^{4, \alpha}\left(\mathbb{R}^{4}\right)} \leq c_{\kappa}(1-\gamma)\left\|\left(h_{1}^{2}, h_{2}^{2}\right)-\left(k_{1}^{2}, k_{2}^{2}\right)\right\|_{\mathcal{C}_{\mu}^{4, \alpha}\left(\mathbb{R}^{4}\right) \times \mathcal{C}_{\mu}^{4, \alpha}\left(\mathbb{R}^{4}\right)}, \\
\left\|\mathcal{M}_{2}\left(h_{1}^{2}, h_{2}^{2}\right)-\mathcal{M}_{2}\left(k_{1}^{2}, k_{2}^{2}\right)\right\|_{\mathcal{C}_{\mu}^{4, \alpha}\left(\mathbb{R}^{4}\right)} \leq c_{\kappa}(1-\xi)\left\|\left(h_{1}^{2}, h_{2}^{2}\right)-\left(k_{1}^{2}, k_{2}^{2}\right)\right\|_{\mathcal{C}_{\mu}^{4, \alpha}\left(\mathbb{R}^{4}\right) \times \mathcal{C}_{\mu}^{4, \alpha}\left(\mathbb{R}^{4}\right)}
\end{gathered}
$$


provided $\left(h_{1}^{2}, h_{2}^{2}\right),\left(k_{1}^{2}, k_{2}^{2}\right)$ in $\mathcal{C}_{\mu}^{4, \alpha}\left(\mathbb{R}^{4}\right) \times \mathcal{C}_{\mu}^{4, \alpha}\left(\mathbb{R}^{4}\right)$ satisfying

$$
\left\|\left(h_{1}^{2}, h_{2}^{2}\right)\right\|_{\mathcal{C}_{\mu}^{4, \alpha}\left(\mathbb{R}^{4}\right) \times \mathcal{C}_{\mu}^{4, \alpha}\left(\mathbb{R}^{4}\right)} \leq 2 c_{\kappa} r_{\varepsilon}^{2} \quad \text { and } \quad\left\|\left(k_{1}^{2}, k_{2}^{2}\right)\right\|_{\mathcal{C}_{\mu}^{4, \alpha}\left(\mathbb{R}^{4}\right) \times \mathcal{C}_{\mu}^{4, \alpha}\left(\mathbb{R}^{4}\right)} \leq 2 c_{\kappa} r_{\varepsilon}^{2} .
$$

Proof. The proof of the first and the second estimates follows from the asymptotic behavior of $H^{\text {int }}$ together with the assumption on the norms of $\varphi_{j}^{2}$ and $\psi_{j}^{2}$ given by 3.20 and it follows from the estimate of $H^{\text {int }}$, given by Lemma 3.7 , that

$$
\left\|H_{\varphi_{j}^{2}, \psi_{j}^{2}}^{\mathrm{int}}\left(\frac{r}{R_{\varepsilon}^{2}} \cdot\right)\right\|_{\mathcal{C}^{4, \alpha}\left(\bar{B}_{2}(0)-B_{1}(0)\right)} \leq C r^{2}\left(R_{\varepsilon}^{2}\right)^{-2}\left(\left\|\varphi_{j}^{2}\right\|_{\mathcal{C}^{4, \alpha}\left(S^{3}\right)}+\left\|\psi_{j}^{2}\right\|_{\mathcal{C}^{2, \alpha}\left(S^{3}\right)}\right)
$$

for all $r \leq R_{\varepsilon}^{2} / 2$. Then by 3.20 , we get

$$
\left\|H_{\varphi_{j}^{2}, \psi_{j}^{2}}^{\mathrm{int}}\left(\frac{r}{R_{\varepsilon}^{2}} \cdot\right)\right\|_{\mathcal{C}^{4, \alpha}\left(\bar{B}_{2}(0)-B_{1}(0)\right)} \leq c_{\kappa} \varepsilon^{2} r^{2} .
$$

On the other hand, using 1.6 we obtain

$$
\begin{aligned}
& \sup _{r \leq R_{\varepsilon}^{2}} r^{4-\mu}\left|\mathcal{T}_{3}(0,0)\right| \\
\leq & \sup _{r \leq R_{\varepsilon}^{2}} \frac{384 r^{4-\mu}}{\left(1+r^{2}\right)^{4}}\left|e^{\frac{(1-\gamma)(\gamma+\xi-1)}{(2-\gamma-\xi)}\left(\frac{1}{\gamma} G\left(\frac{\varepsilon x}{\tau_{2}}, x^{1}\right)-\frac{1}{\xi} G\left(\frac{\varepsilon x}{\tau_{2}}, x^{3}\right)\right)} e^{\gamma H_{\varphi_{1}^{2}, \psi_{1}^{2}}^{\mathrm{int}}+(1-\gamma) H_{\varphi_{2}^{2}}^{\mathrm{int}}, \psi_{2}^{2}}-1\right| \\
\leq & c \sup _{r \leq R_{\varepsilon}^{2}} \frac{384 r^{4-\mu} r^{2} \varepsilon^{2}}{\left(1+r^{2}\right)^{4}} .
\end{aligned}
$$

Making use of Proposition 3.2 together with (3.4), for $\mu \in(1,2)$, we get that there exists $c_{\kappa}$ such that

$$
\left\|\mathcal{N}_{2}(0,0)\right\|_{\mathcal{C}_{\mu}^{4, \alpha}\left(\mathbb{R}^{4}\right)} \leq c_{\kappa} r_{\varepsilon}^{2}
$$

For the second estimate, we use the same techniques to prove

$$
\left\|\mathcal{M}_{2}(0,0)\right\|_{\mathcal{C}_{\mu}^{4, \alpha}\left(\mathbb{R}^{4}\right)} \leq c_{\kappa} r_{\varepsilon}^{2}
$$

To derive the third estimate, for $\left(h_{1}^{2}, h_{2}^{2}\right),\left(k_{1}^{2}, k_{2}^{2}\right)$ verifying 4.6$)$, we have

$$
\begin{aligned}
& \sup _{r \leq R_{\varepsilon}^{2}} r^{4-\mu}\left|\mathcal{T}_{3}\left(h_{1}^{2}, h_{2}^{2}\right)-\mathcal{T}_{3}\left(k_{1}^{2}, k_{2}^{2}\right)\right| \\
& \leq \sup _{r \leq R_{\varepsilon}^{2}} \frac{384 r^{4-\mu}}{\left(1+r^{2}\right)^{4}} \mid e^{\frac{(1-\gamma)(\gamma+\xi-1)}{(2-\gamma-\xi)}\left(\frac{1}{\gamma} G\left(\frac{\varepsilon x}{\tau_{2}}, x^{1}\right)-\frac{1}{\xi} G\left(\frac{\varepsilon x}{\tau_{2}}, x^{3}\right)\right)} e^{\gamma h_{1}^{2}+\gamma H_{\varphi_{1}^{2}, \psi_{1}^{2}}^{\mathrm{int}}+(1-\gamma) h_{2}^{2}+(1-\gamma) H_{\varphi_{2}^{2}, \psi_{2}^{2}}^{\mathrm{int}}}-h_{1}^{2} \\
& -e^{\frac{(1-\gamma)(\gamma+\xi-1)}{(2-\gamma-\xi)}\left(\frac{1}{\gamma} G\left(\frac{\varepsilon x}{\tau_{2}}, x^{1}\right)-\frac{1}{\xi} G\left(\frac{\varepsilon x}{\tau_{2}}, x^{3}\right)\right)} e^{\gamma k_{1}^{2}+\gamma H_{\varphi_{1}^{2}, \psi_{1}^{2}}^{\mathrm{int}}+(1-\gamma) k_{2}^{2}+(1-\gamma) H_{\varphi_{2}^{2}, \psi_{2}^{2}}^{\mathrm{int}}+k_{1}^{2} \mid} \\
& \leq c \sup _{r \leq R_{\varepsilon}^{2}} \frac{384 r^{4-\mu}}{\left(1+r^{2}\right)^{4}}\left|(\gamma-1)\left(h_{1}^{2}-k_{1}^{2}\right)+(1-\gamma)\left(h_{2}^{2}-k_{2}^{2}\right)\right| \\
& \leq c \sup _{r \leq R_{\varepsilon}^{2}} \frac{384 r^{4-\mu}}{\left(1+r^{2}\right)^{4}}(1-\gamma)\left[r^{\mu}\left\|h_{1}^{2}-k_{1}^{2}\right\|_{\mathcal{C}_{\mu}^{4, \alpha}}+r^{\mu}\left\|h_{2}^{2}-k_{2}^{2}\right\|_{\mathcal{C}_{\mu}^{4, \alpha}}\right]
\end{aligned}
$$


We conclude that

$$
\left\|\mathcal{N}_{2}\left(h_{1}^{2}, h_{2}^{2}\right)-\mathcal{N}_{2}\left(k_{1}^{2}, k_{2}^{2}\right)\right\|_{\mathcal{C}_{\mu}^{4, \alpha}\left(\mathbb{R}^{4}\right)} \leq c_{\kappa}(1-\gamma)\left\|\left(h_{1}^{2}, h_{2}^{2}\right)-\left(k_{1}^{2}, k_{2}^{2}\right)\right\|_{\mathcal{C}_{\mu}^{4, \alpha}\left(\mathbb{R}^{4}\right) \times \mathcal{C}_{\mu}^{4, \alpha}\left(\mathbb{R}^{4}\right)} .
$$

Similarly, we get

$$
\left\|\mathcal{M}_{2}\left(h_{1}^{2}, h_{2}^{2}\right)-\mathcal{M}_{2}\left(k_{1}^{2}, k_{2}^{2}\right)\right\|_{\mathcal{C}_{\mu}^{4, \alpha}\left(\mathbb{R}^{4}\right)} \leq c_{\kappa}(1-\xi)\left\|\left(h_{1}^{2}, h_{2}^{2}\right)-\left(k_{1}^{2}, k_{2}^{2}\right)\right\|_{\mathcal{C}_{\mu}^{4, \alpha}\left(\mathbb{R}^{4}\right) \times \mathcal{C}_{\mu}^{4, \alpha}\left(\mathbb{R}^{4}\right)}
$$

Then there exist $\gamma_{0}$ and $\xi_{0} \in(0,1)$ such that $c_{\kappa}(1-\gamma) \leq 1 / 2$ and $c_{\kappa}(1-\xi) \leq 1 / 2$ for all $\gamma \in\left(\gamma_{0}, 1\right)$ and $\xi \in\left(\xi_{0}, 1\right)$. Therefore (4.7) and (4.8) are enough to show that

$$
\left(h_{1}^{2}, h_{2}^{2}\right) \mapsto\left(\mathcal{N}_{2}\left(h_{1}^{2}, h_{2}^{2}\right), \mathcal{M}_{2}\left(h_{1}^{2}, h_{2}^{2}\right)\right)
$$

is a contraction from the ball

$$
\left\{\left(h_{1}^{2}, h_{2}^{2}\right) \in \mathcal{C}_{\mu}^{4, \alpha}\left(\mathbb{R}^{4}\right) \times \mathcal{C}_{\mu}^{4, \alpha}\left(\mathbb{R}^{4}\right):\left\|\left(h_{1}^{2}, h_{2}^{2}\right)\right\|_{\mathcal{C}_{\mu}^{4, \alpha}\left(\mathbb{R}^{4}\right) \times \mathcal{C}_{\mu}^{4, \alpha}\left(\mathbb{R}^{4}\right)} \leq 2 c_{\kappa} r_{\varepsilon}^{2}\right\}
$$

into itself. Then applying a contraction mapping argument, we obtain the following proposition.

Proposition 4.4. Given $\kappa>0, \mu \in(1,2), \gamma_{0} \in(0,1)$ and $\xi_{0} \in(0,1)$, there exist $\varepsilon_{\kappa}>0$ and $c_{\kappa}>0$ such that for all $\varepsilon \in\left(0, \varepsilon_{\kappa}\right), \gamma \in\left(\gamma_{0}, 1\right)$ and $\xi \in\left(\xi_{0}, 1\right)$, for all $\tau_{2}$ in some fixed compact subset of $\left[\tau_{2}^{-}, \tau_{2}^{+}\right] \subset(0, \infty)$ and for $\varphi_{j}^{2}$ and $\psi_{j}^{2}$ satisfying (3.5) and (3.20), there exists a unique $\left(h_{1}^{2}, h_{2}^{2}\right)\left(:=\left(h_{1, \varepsilon, \tau_{2}, \varphi_{1}^{2}, \psi_{1}^{2}}, h_{2, \varepsilon, \tau_{2}, \varphi_{2}^{2}, \psi_{2}^{2}}\right)\right)$ solution of 4.5 such that

$$
\left\|\left(h_{1}^{2}, h_{2}^{2}\right)\right\|_{C_{\mu}^{4, \alpha}\left(\mathbb{R}^{4}\right) \times C_{\mu}^{4, \alpha}\left(\mathbb{R}^{4}\right)} \leq 2 c_{\kappa} r_{\varepsilon}^{2} .
$$

Hence

$$
\begin{aligned}
v_{1}(x):= & \bar{u}\left(x-x^{2}\right)+\frac{1-\gamma}{\gamma(2-\gamma-\xi)} G\left(\frac{\varepsilon x}{\tau_{2}}, x^{1}\right)-\frac{1-\gamma}{\xi(2-\gamma-\xi)} G\left(\frac{\varepsilon x}{\tau_{2}}, x^{3}\right) \\
& +h_{1}^{2}(x)+H^{\operatorname{int}}\left(\varphi_{1}^{2}, \psi_{1}^{2} ; \frac{x-x^{2}}{R_{\varepsilon}^{2}}\right) \\
v_{2}(x):= & \bar{u}\left(x-x^{2}\right)-\frac{1-\xi}{\gamma(2-\gamma-\xi)} G\left(\frac{\varepsilon x}{\tau_{2}}, x^{1}\right)+\frac{1-\xi}{\xi(2-\gamma-\xi)} G\left(\frac{\varepsilon x}{\tau_{2}}, x^{3}\right) \\
& +h_{2}^{2}(x)+H^{\operatorname{int}}\left(\varphi_{2}^{2}, \psi_{2}^{2} ; \frac{x-x^{2}}{R_{\varepsilon}^{2}}\right)
\end{aligned}
$$

solves 4.2 in $B_{R_{\varepsilon}^{2}}\left(x^{2}\right)$.

Remark also that the functions $\left(h_{1}^{i}, h_{2}^{i}\right)\left(:=\left(h_{1, \varepsilon, \tau_{i}, \varphi_{1}^{i}, \psi_{1}^{i}}^{i}, h_{2, \varepsilon, \tau_{i}, \varphi_{2}^{i}, \psi_{2}^{i}}^{i}\right)\right)$, for $i \in\{1,2,3\}$, depend continuously on the parameter $\tau_{i}$. 


\subsection{The nonlinear exterior problem}

By the same arguments as in the proof of Theorem 1.5, exterior problem, we obtain the following proposition.

Proposition 4.5. Given $\kappa>0$, there exists $\varepsilon_{\kappa}>0$ (depending on $\kappa$ ) such that for any $\varepsilon \in\left(0, \varepsilon_{\kappa}\right), \lambda_{i}$ and $\widetilde{x}^{i}$ satisfying (3.29) and functions $\widetilde{\varphi}_{j}^{i}$ and $\widetilde{\psi}_{j}^{i}$ satisfying (3.6) and (3.28),

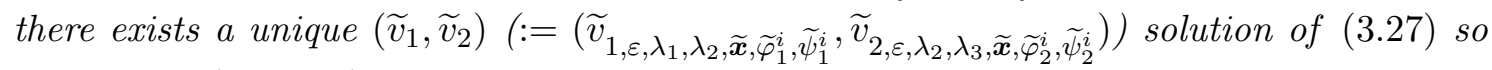
that for $v_{k}(k=1,2)$ defined by

$$
\begin{aligned}
v_{1}(x):= & \frac{1+\lambda_{1}}{\gamma} G\left(x, \widetilde{x}^{1}\right)+\left(1+\lambda_{2}\right) G\left(x, \widetilde{x}^{2}\right) \\
& +\sum_{i=1}^{3} \chi_{r_{0}}\left(x-\widetilde{x}^{i}\right) H^{\operatorname{ext}}\left(\widetilde{\varphi}_{1}^{i}, \widetilde{\psi}_{1}^{i} ; \frac{x-\widetilde{x}^{i}}{r_{\varepsilon}}\right)+\widetilde{v}_{1}(x), \\
v_{2}(x):= & \frac{1+\lambda_{3}}{\xi} G\left(x, \widetilde{x}^{3}\right)+\left(1+\lambda_{2}\right) G\left(x, \widetilde{x}^{2}\right) \\
& +\sum_{i=1}^{3} \chi_{r_{0}}\left(x-\widetilde{x}^{i}\right) H^{\operatorname{ext}}\left(\widetilde{\varphi}_{2}^{i}, \widetilde{\psi}_{2}^{i} ; \frac{x-\widetilde{x}^{i}}{r_{\varepsilon}}\right)+\widetilde{v}_{2}(x)
\end{aligned}
$$

solves (3.24) in $\bar{\Omega}_{r_{\varepsilon}}(\widetilde{\boldsymbol{x}})$. In addition, we have

$$
\left\|\left(\widetilde{v}_{1}, \widetilde{v}_{2}\right)\right\|_{C_{\nu}^{4, \alpha}\left(\bar{\Omega}^{*}(\widetilde{\boldsymbol{x}})\right)} \leq 2 c_{\kappa} r_{\varepsilon}^{2} .
$$

\subsection{The nonlinear Cauchy-data matching}

We will gather the results of the previous sections. Using the previous notations, assume that $\widetilde{\boldsymbol{x}}:=\left(\widetilde{x}^{1}, \widetilde{x}^{2}, \widetilde{x}^{3}\right) \in \Omega^{3}$ are given close to $\boldsymbol{x}:=\left(x^{1}, x^{2}, x^{3}\right)$. Assume also that

$$
\boldsymbol{\tau}:=\left(\tau_{1}, \tau_{2}, \tau_{3}\right) \in\left[\tau_{1}^{-}, \tau_{1}^{+}\right] \times\left[\tau_{2}^{-}, \tau_{2}^{+}\right] \times\left[\tau_{3}^{-}, \tau_{3}^{+}\right] \subset(0, \infty)^{3}
$$

are given (the values of $\tau_{l}^{-}$and $\tau_{l}^{+}$, for $l=1,2,3$ will be fixed later). First, we consider some set of boundary data $\varphi^{i}:=\left(\varphi_{1}^{i}, \varphi_{2}^{i}\right) \in\left(\mathcal{C}^{4, \alpha}\left(S^{3}\right)\right)^{2}$ and $\psi^{i}:=\left(\psi_{1}^{i}, \psi_{2}^{i}\right) \in\left(\mathcal{C}^{2, \alpha}\left(S^{3}\right)\right)^{2}$. According to the result of Propositions 3.10, 3.11 and 3.13 and provided $\varepsilon \in\left(0, \varepsilon_{\kappa}\right)$, we can find, $u_{\text {int }}:=\left(u_{\text {int }, 1}, u_{\text {int }, 2}\right)$ a solution of $(3.7)$ in $B_{r_{\varepsilon}}\left(\widetilde{x}^{1}\right) \cup B_{r_{\varepsilon}}\left(\widetilde{x}^{2}\right) \cup B_{r_{\varepsilon}}\left(\widetilde{x}^{3}\right)$, which can be decomposed as

$$
u_{\text {int }, 1}(x):= \begin{cases}\frac{1}{\gamma} u_{\varepsilon, \tau_{1}}\left(x-\widetilde{x}^{1}\right)-\frac{1-\gamma}{\gamma} G\left(x, \widetilde{x}^{2}\right)-\frac{1-\gamma}{\gamma \xi} G\left(x, \widetilde{x}^{3}\right) & \\ \quad-\frac{\ln \gamma}{\gamma}+H_{\varphi_{1}^{1}, \psi_{1}^{1}}^{\mathrm{int}}\left(\frac{x-\widetilde{x}^{1}}{r_{\varepsilon}}\right)+h_{1}^{1}\left(\frac{R_{\varepsilon}^{1}\left(x-\widetilde{x}^{1}\right)}{r_{\varepsilon}}\right) & \text { in } B_{r_{\varepsilon}}\left(\widetilde{x}^{1}\right), \\ u_{\varepsilon, \tau_{2}}\left(x-\widetilde{x}^{2}\right)+\frac{1-\gamma}{\gamma(2-\gamma-\xi)} G\left(\frac{\varepsilon x}{\tau_{2}}, \widetilde{x}^{1}\right)-\frac{1-\gamma}{\xi(2-\gamma-\xi)} G\left(\frac{\varepsilon x}{\tau_{2}}, \widetilde{x}^{3}\right) & \\ \quad+H_{\varphi_{1}^{2}, \psi_{1}^{2}}^{\text {int }}\left(\frac{x-\widetilde{x}^{2}}{r_{\varepsilon}}\right)+h_{1}^{2}\left(\frac{R_{\varepsilon}^{2}\left(x-\widetilde{x}^{2}\right)}{r_{\varepsilon}}\right) & \text { in } B_{r_{\varepsilon}}\left(\widetilde{x}^{2}\right), \\ \frac{1}{\gamma} G\left(x, \widetilde{x}^{1}\right)+G\left(x, \widetilde{x}^{2}\right)+H_{\varphi_{1}^{3}, \psi_{1}^{3}}^{\text {int }}\left(\frac{x-\widetilde{x}^{3}}{r_{\varepsilon}}\right)+h_{1}^{3}\left(\frac{R_{\varepsilon}^{3}\left(x-\widetilde{x}^{3}\right)}{r_{\varepsilon}}\right) & \text { in } B_{r_{\varepsilon}}\left(\widetilde{x}^{3}\right)\end{cases}
$$


and

$$
u_{\text {int }, 2}(x):= \begin{cases}\frac{1}{\xi} G\left(x, \widetilde{x}^{3}\right)+G\left(x, \widetilde{x}^{2}\right)+H_{\varphi_{2}^{1}, \psi_{2}^{1}}^{\text {int }}\left(\frac{x-\widetilde{x}^{1}}{r_{\varepsilon}}\right)+h_{2}^{1}\left(\frac{R_{\varepsilon}^{1}\left(x-\widetilde{x}^{1}\right)}{r_{\varepsilon}}\right) & \text { in } B_{r_{\varepsilon}}\left(\widetilde{x}^{1}\right), \\ u_{\varepsilon, \tau_{2}}\left(x-\widetilde{x}^{2}\right)-\frac{1-\xi}{\gamma(2-\gamma-\xi)} G\left(\frac{\varepsilon x}{\tau_{2}}, \widetilde{x}^{1}\right)+\frac{1-\xi}{\xi(2-\gamma-\xi)} G\left(\frac{\varepsilon x}{\tau_{2}}, \widetilde{x}^{3}\right) & \\ \quad+H_{\varphi_{2}^{2}, \psi_{2}^{2}}^{\text {int }}\left(\frac{x-\widetilde{x}^{2}}{r_{\varepsilon}}\right)+h_{2}^{2}\left(\frac{R_{\varepsilon}^{2}\left(x-\widetilde{x}^{2}\right)}{r_{\varepsilon}}\right) & \text { in } B_{r_{\varepsilon}}\left(\widetilde{x}^{2}\right), \\ \frac{1}{\xi} u_{\varepsilon, \tau_{3}}\left(x-\widetilde{x}^{3}\right)-\frac{1-\xi}{\xi} G\left(x, \widetilde{x}^{2}\right)-\frac{1-\xi}{\gamma \xi} G\left(x, \widetilde{x}^{1}\right) & \\ -\frac{\ln \xi}{\xi}+H_{\varphi_{2}^{3}, \psi_{2}^{3}}^{\text {int }}\left(\frac{x-\widetilde{x}^{3}}{r_{\varepsilon}}\right)+h_{2}^{3}\left(\frac{R_{\varepsilon}^{3}\left(x-\widetilde{x}^{3}\right)}{r_{\varepsilon}}\right) & \text { in } B_{r_{\varepsilon}}\left(\widetilde{x}^{3}\right),\end{cases}
$$

where for $i \in\{1,2,3\}$ and $j \in\{1,2\}, R_{\varepsilon}^{i}=\tau_{i} \frac{r_{\varepsilon}}{\varepsilon}$ and the functions $h_{j}^{i}$ satisfy

$$
\left\|\left(h_{1}^{1}, h_{2}^{1}\right)\right\|_{\mathcal{C}_{\mu}^{4, \alpha}\left(\mathbb{R}^{4}\right) \times \mathcal{C}_{\delta}^{4, \alpha}\left(\mathbb{R}^{4}\right)} \leq 2 c_{\kappa} r_{\varepsilon}^{2}, \quad\left\|\left(h_{1}^{2}, h_{2}^{2}\right)\right\|_{\left(\mathcal{C}_{\mu}^{4, \alpha}\left(\mathbb{R}^{4}\right)\right)^{2}} \leq 2 c_{\kappa} r_{\varepsilon}^{2}
$$

and

$$
\left\|\left(h_{1}^{3}, h_{2}^{3}\right)\right\|_{\mathcal{C}_{\delta}^{4, \alpha}\left(\mathbb{R}^{4}\right) \times \mathcal{C}_{\mu}^{4, \alpha}\left(\mathbb{R}^{4}\right)} \leq 2 c_{\kappa} r_{\varepsilon}^{2} .
$$

Similarly, given some boundary data $\widetilde{\varphi}_{j}^{i} \in C^{4, \alpha}\left(S^{3}\right), \widetilde{\psi}_{j}^{i} \in C^{2, \alpha}\left(S^{3}\right)$ satisfying (3.6), $\left(\lambda_{1}, \lambda_{2}, \lambda_{3}\right) \in \mathbb{R}^{3}$ satisfying (3.29), provided $\varepsilon \in\left(0, \varepsilon_{\kappa}\right)$, by Proposition 3.15, we find a solution $u_{\text {ext }}:=\left(u_{\text {ext }, 1}, u_{\text {ext }, 2}\right)$ of $(3.7)$ in $\left.\bar{\Omega} \backslash\left(B_{r_{\varepsilon}}\left(\widetilde{x}^{1}\right) \cup B_{r_{\varepsilon}}\left(\widetilde{x}^{2}\right)\right) \cup B_{r_{\varepsilon}}\left(\widetilde{x}^{3}\right)\right)$ which can be decomposed as

$$
\begin{aligned}
u_{\text {ext }, 1}(x):= & \frac{1+\lambda_{1}}{\gamma} G\left(x, \widetilde{x}^{1}\right)+\left(1+\lambda_{2}\right) G\left(x, \widetilde{x}^{2}\right) \\
& +\sum_{i=1}^{3} \chi_{r_{0}}\left(x-\widetilde{x}^{i}\right) H^{\operatorname{ext}}\left(\widetilde{\varphi}_{1}^{i}, \widetilde{\psi}_{1}^{i} ; \frac{x-\widetilde{x}^{i}}{r_{\varepsilon}}\right)+\widetilde{v}_{1}(x), \\
u_{\text {ext }, 2}(x):= & \frac{1+\lambda_{3}}{\xi} G\left(x, \widetilde{x}^{3}\right)+\left(1+\lambda_{2}\right) G\left(x, \widetilde{x}^{2}\right) \\
& +\sum_{i=1}^{3} \chi_{r_{0}}\left(x-\widetilde{x}^{i}\right) H^{\operatorname{ext}}\left(\widetilde{\varphi}_{2}^{i}, \widetilde{\psi}_{2}^{i} ; \frac{x-\widetilde{x}^{i}}{r_{\varepsilon}}\right)+\widetilde{v}_{2}(x)
\end{aligned}
$$

with $\widetilde{v}_{1}, \widetilde{v}_{2} \in C_{\nu}^{4, \alpha}\left(\bar{\Omega}^{*}(\widetilde{\boldsymbol{x}})\right)$ satisfying

$$
\left\|\left(\widetilde{v}_{1}, \widetilde{v}_{2}\right)\right\|_{\left(C_{\nu}^{4, \alpha}\left(\bar{\Omega}^{*}(\widetilde{\boldsymbol{x}})\right)\right)^{2}} \leq 2 c_{\kappa} r_{\varepsilon}^{2} .
$$

It remains to determine the parameters and the boundary data in such a way that the function equal to $u_{\text {int }}$ in $B_{r_{\varepsilon}}\left(\widetilde{x}^{1}\right) \cup B_{r_{\varepsilon}}\left(\widetilde{x}^{2}\right) \cup B_{r_{\varepsilon}}\left(\widetilde{x}^{3}\right)$ and equal to $u_{\text {ext }}$ in $\bar{\Omega}_{r_{\varepsilon}}(\widetilde{\boldsymbol{x}})$ is a smooth function. This amounts to find the boundary data and the parameters so that, for each $j=1,2$,

$$
\begin{array}{rlrl}
u_{\mathrm{int}, j} & =u_{\mathrm{ext}, j}, & \partial_{r} u_{\mathrm{int}, j} & =\partial_{r} u_{\mathrm{ext}, j}, \\
\Delta u_{\mathrm{int}, j} & =\Delta u_{\mathrm{ext}, j}, \quad \partial_{r} \Delta u_{\mathrm{int}, j}=\partial_{r} \Delta u_{\mathrm{ext}, j}
\end{array}
$$

on $\partial B_{r_{\varepsilon}}\left(\widetilde{x}^{1}\right), \partial B_{r_{\varepsilon}}\left(\widetilde{x}^{2}\right)$ and $\partial B_{r_{\varepsilon}}\left(\widetilde{x}^{3}\right)$. 
Suppose that 4.9 is verified, this provides that for each $\varepsilon$ small enough $u_{\varepsilon} \in \mathcal{C}^{4, \alpha}$ (which is obtained by patching together the functions $u_{\text {int }}$ and the function $u_{\text {ext }}$ ), a weak solution of our system and elliptic regularity theory implies that this solution is in fact smooth. That will complete the proof since, as $\varepsilon$ tends to 0 , the sequence of solutions we have obtain satisfies the required singular limit behaviors.

Before we proceed, the following remarks are due. First it will be convenient to observe that the function $u_{\varepsilon, \tau_{i}}$ can be expanded as

$$
u_{\varepsilon, \tau_{i}}(x)=-4 \ln \tau_{i}-8 \ln |x|+\mathcal{O}\left(\frac{\varepsilon^{2} \tau_{i}^{-2}}{|x|^{2}}\right) \quad \text { on } \partial B_{r_{\varepsilon}}(0) .
$$

- On $\partial B_{r_{\varepsilon}}\left(\widetilde{x}^{1}\right)$, according to the proof of Theorem 1.5 and since when $\varepsilon$ tend to 0 , it is enough to choose $\tau_{1}^{-}$and $\tau_{1}^{+}$in such a way that

$$
4 \ln \left(\tau_{1}^{-}\right)<-\ln \gamma-\mathcal{E}_{1}\left(x^{1}, \boldsymbol{x}\right)<4 \ln \left(\tau_{1}^{+}\right)
$$

where

$$
\mathcal{E}_{1}(\cdot, \widetilde{\boldsymbol{x}}):=H\left(\cdot, \widetilde{x}^{1}\right)+G\left(\cdot, \widetilde{x}^{2}\right)+\frac{1-\gamma}{\xi} G\left(\cdot, \widetilde{x}^{3}\right) .
$$

Also using the fact that

$$
\begin{array}{ll}
\varphi_{1}^{1}=\varphi_{1,0}^{1}+\varphi_{1,1}^{1}+\varphi_{1}^{1, \perp}, & \psi_{1}^{1}=8 \varphi_{1,0}^{1}+12 \varphi_{1,1}^{1}+\psi_{1}^{1, \perp} \\
\widetilde{\varphi}_{1}^{1}=\widetilde{\varphi}_{1,0}^{1}+\widetilde{\varphi}_{1,1}^{1}+\widetilde{\varphi}_{1}^{1, \perp}, & \widetilde{\psi}_{1}^{1}=\widetilde{\psi}_{1,1}^{1}+\widetilde{\psi}_{1}^{1, \perp}
\end{array}
$$

where $\varphi_{1,0}^{1}, \widetilde{\varphi}_{1,0}^{1} \in \mathbb{E}_{0}=\mathbb{R}$ are constant on $S^{3}, \varphi_{1,1}^{1}, \widetilde{\varphi}_{1,1}^{1}, \widetilde{\psi}_{1,1}^{1}$ belong to $\mathbb{E}_{1}=\operatorname{Span}\left\{e_{1}, e_{2}\right.$, $\left.e_{3}, e_{4}\right\}$ and $\varphi_{1}^{1, \perp}, \widetilde{\varphi}_{1}^{1, \perp}, \psi_{1}^{1, \perp}, \widetilde{\psi}_{1}^{1, \perp}$ are $L^{2}\left(S^{3}\right)$ orthogonal to $\mathbb{E}_{0}$ and $\mathbb{E}_{1}$. We can prove that

$$
\begin{array}{rlrl}
\left(u_{\mathrm{int}, 1}-u_{\mathrm{ext}, 1}\right)\left(\widetilde{x}^{1}+r_{\varepsilon} \cdot\right) & =0, & \partial_{r}\left(u_{\mathrm{int}, 1}-u_{\mathrm{ext}, 1}\right)\left(\widetilde{x}^{1}+r_{\varepsilon} \cdot\right) & =0, \\
\Delta\left(u_{\mathrm{int}, 1}-u_{\mathrm{ext}, 1}\right)\left(\widetilde{x}^{1}+r_{\varepsilon} \cdot\right) & =0, \quad \partial_{r} \Delta\left(u_{\mathrm{int}, 1}-u_{\mathrm{ext}, 1}\right)\left(\widetilde{x}^{1}+r_{\varepsilon} \cdot\right) & =0
\end{array}
$$

on $S^{3}$ yield to

$$
T_{1, \varepsilon}^{1}=\left(t_{1}, \lambda_{1}, \varphi_{1,0}^{1}, \widetilde{\varphi}_{1,0}^{1}, \varphi_{1,1}^{1}, \widetilde{\varphi}_{1,1}^{1}, \widetilde{\psi}_{1,1}^{1}, \bar{\nabla} \mathcal{E}_{1}\left(\widetilde{x}^{1}, \widetilde{\boldsymbol{x}}\right), \varphi_{1}^{1, \perp}, \widetilde{\varphi}_{1}^{1, \perp}, \psi_{1}^{1, \perp}, \widetilde{\psi}_{1}^{1, \perp}\right)=\mathcal{O}\left(r_{\varepsilon}^{2}\right),
$$

where

$$
t_{1}=\frac{1}{\ln r_{\varepsilon}}\left[4 \ln \tau_{1}+\ln \gamma+\mathcal{E}_{1}\left(\widetilde{x}^{1}, \widetilde{\boldsymbol{x}}\right)\right] .
$$

Finally, using the fact that

$$
\begin{array}{ll}
\varphi_{2}^{1}=\varphi_{2,0}^{1}+\varphi_{2,1}^{1}+\varphi_{2}^{1, \perp}, & \psi_{2}^{1}=8 \varphi_{2,0}^{1}+12 \varphi_{2,1}^{1}+\psi_{2}^{1, \perp}, \\
\widetilde{\varphi}_{2}^{1}=\widetilde{\varphi}_{2,0}^{1}+\widetilde{\varphi}_{2,1}^{1}+\widetilde{\varphi}_{2}^{1, \perp}, & \widetilde{\psi}_{2}^{1}=\widetilde{\psi}_{2,1}^{1}+\widetilde{\psi}_{2}^{1, \perp}
\end{array}
$$


with $\varphi_{2,0}^{1}, \widetilde{\varphi}_{2,0}^{1} \in \mathbb{E}_{0}, \varphi_{2,1}^{1}, \widetilde{\varphi}_{2,1}^{1}, \widetilde{\psi}_{2,1}^{1} \in \mathbb{E}_{1}$ and $\varphi_{2}^{1, \perp}, \widetilde{\varphi}_{2}^{1, \perp}, \psi_{2}^{1, \perp}, \widetilde{\psi}_{2}^{1, \perp}$ belong to $\left(L^{2}\left(S^{3}\right)\right)^{\perp}$. We can prove that

$$
\begin{aligned}
& \left(u_{\mathrm{int}, 2}-u_{\mathrm{ext}, 2}\right)\left(\widetilde{x}^{1}+r_{\varepsilon} \cdot\right)=0, \quad \partial_{r}\left(u_{\mathrm{int}, 2}-u_{\mathrm{ext}, 2}\right)\left(\widetilde{x}^{1}+r_{\varepsilon} \cdot\right)=0, \\
& \Delta\left(u_{\mathrm{int}, 2}-u_{\mathrm{ext}, 2}\right)\left(\widetilde{x}^{1}+r_{\varepsilon} \cdot\right)=0, \quad \partial_{r} \Delta\left(u_{\mathrm{int}, 2}-u_{\mathrm{ext}, 2}\right)\left(\widetilde{x}^{1}+r_{\varepsilon} \cdot\right)=0
\end{aligned}
$$

on $S^{3}$ yield to

$$
T_{2, \varepsilon}^{1}=\left(\varphi_{2,0}^{1}, \widetilde{\varphi}_{2,0}^{1}, \varphi_{2,1}^{1}, \widetilde{\varphi}_{2,1}^{1}, \widetilde{\psi}_{2,1}^{1}, \varphi_{2}^{1, \perp}, \widetilde{\varphi}_{2}^{1, \perp}, \psi_{2}^{1, \perp}, \widetilde{\psi}_{2}^{1, \perp}\right)=\mathcal{O}\left(r_{\varepsilon}^{2}\right)
$$

As usual, the terms $\mathcal{O}\left(r_{\varepsilon}^{2}\right)$ depend nonlinearly on all the variables on the left side, but is bounded (in the appropriate norm) by a constant (independent of $\varepsilon$ and $\kappa$ ) times $r_{\varepsilon}^{2}$, provided $\varepsilon \in\left(0, \varepsilon_{\kappa}\right)$.

- On $\partial B_{r_{\varepsilon}}\left(\widetilde{x}^{2}\right)$, we have

$$
\begin{aligned}
\left(u_{\mathrm{int}, 1}-u_{\mathrm{ext}, 1}\right)(x)= & -4 \ln \tau_{2}+8 \lambda_{2} \ln \left|x-\widetilde{x}^{2}\right|+h_{1}^{2}\left(R_{\varepsilon}^{2} \frac{x-\widetilde{x}^{2}}{r_{\varepsilon}}\right) \\
& +H^{\operatorname{int}}\left(\varphi_{1}^{2}, \psi_{1}^{2} ; \frac{x-\widetilde{x}^{2}}{r_{\varepsilon}}\right)-H^{\mathrm{ext}}\left(\widetilde{\varphi}_{1}^{2}, \widetilde{\psi}_{1}^{2} ; \frac{x-\widetilde{x}^{2}}{r_{\varepsilon}}\right) \\
& -\left[(2-\gamma-\xi) H\left(x, \widetilde{x}^{2}\right)+\frac{1-\xi}{\gamma} G\left(x, \widetilde{x}^{1}\right)+\frac{1-\gamma}{\xi} G\left(x, \widetilde{x}^{3}\right)\right] \\
& +\mathcal{O}\left(\frac{\varepsilon^{2} \tau_{2}^{-2}}{\left|x-\widetilde{x}^{2}\right|^{2}}\right)+\mathcal{O}\left(r_{\varepsilon}^{2}\right)
\end{aligned}
$$

and

$$
\begin{aligned}
\left(u_{\mathrm{int}, 2}-u_{\mathrm{ext}, 2}\right)(x)= & -4 \ln \tau_{2}+8 \lambda_{2} \ln \left|x-\widetilde{x}^{2}\right|+h_{2}^{2}\left(R_{\varepsilon}^{2} \frac{x-\widetilde{x}^{2}}{r_{\varepsilon}}\right) \\
& +H^{\operatorname{int}}\left(\varphi_{2}^{2}, \psi_{2}^{2} ; \frac{x-\widetilde{x}^{2}}{r_{\varepsilon}}\right)-H^{\mathrm{ext}}\left(\widetilde{\varphi}_{2}^{2}, \widetilde{\psi}_{2}^{2} ; \frac{x-\widetilde{x}^{2}}{r_{\varepsilon}}\right) \\
& -\left[(2-\gamma-\xi) H\left(x, \widetilde{x}^{2}\right)+\frac{1-\xi}{\gamma} G\left(x, \widetilde{x}^{1}\right)+\frac{1-\gamma}{\xi} G\left(x, \widetilde{x}^{3}\right)\right] \\
& +\mathcal{O}\left(\frac{\varepsilon^{2} \tau_{2}^{-2}}{\left|x-\widetilde{x}^{2}\right|^{2}}\right)+\mathcal{O}\left(r_{\varepsilon}^{2}\right) .
\end{aligned}
$$

Next, even though all functions are defined on $\partial B_{r_{\varepsilon}}\left(\widetilde{x}^{2}\right)$ in 4.9 , it will be more convenient to solve on $S^{3}$, for $i=1,2$, the following set of equations

$$
\begin{array}{rr}
\left(u_{\mathrm{int}, i}-u_{\mathrm{ext}, i}\right)\left(\widetilde{x}^{2}+r_{\varepsilon} \cdot\right)=0, & \partial_{r}\left(u_{\mathrm{int}, i}-u_{\mathrm{ext}, i}\right)\left(\widetilde{x}^{2}+r_{\varepsilon} \cdot\right)=0, \\
\Delta\left(u_{\mathrm{int}, i}-u_{\mathrm{ext}, i}\right)\left(\widetilde{x}^{2}+r_{\varepsilon} \cdot\right)=0, \quad \partial_{r} \Delta\left(u_{\mathrm{int}, i}-u_{\mathrm{ext}, i}\right)\left(\widetilde{x}^{2}+r_{\varepsilon} \cdot\right)=0 .
\end{array}
$$

Since the boundary data are chosen to satisfy (3.5) or (3.6). We decompose

$$
\begin{array}{ll}
\varphi_{i}^{2}=\varphi_{i, 0}^{2}+\varphi_{i, 1}^{2}+\varphi_{i}^{2, \perp}, & \psi_{i}^{2}=8 \varphi_{i, 0}^{2}+12 \varphi_{i, 1}^{2}+\psi_{i}^{2, \perp}, \\
\widetilde{\varphi}_{i}^{2}=\widetilde{\varphi}_{i, 0}^{2}+\widetilde{\varphi}_{i, 1}^{2}+\widetilde{\varphi}_{i}^{2, \perp}, & \widetilde{\psi}_{i}^{2}=\widetilde{\psi}_{i, 1}^{2}+\widetilde{\psi}_{i}^{2, \perp},
\end{array}
$$


where $\varphi_{i, 0}^{1}, \widetilde{\varphi}_{i, 0}^{1} \in \mathbb{E}_{0}=\mathbb{R}$ are constant on $S^{3}, \varphi_{i, 1}^{1}, \widetilde{\varphi}_{i, 1}^{1}, \widetilde{\psi}_{i, 1}^{1}$ belong to $\mathbb{E}_{1}=\operatorname{Span}\left\{e_{1}, e_{2}\right.$, $\left.e_{3}, e_{4}\right\}$ and $\varphi_{i}^{1, \perp}, \widetilde{\varphi}_{i}^{1, \perp}, \psi_{i}^{1, \perp}, \widetilde{\psi}_{i}^{1, \perp}$ are $L^{2}\left(S^{3}\right)$ orthogonal to $\mathbb{E}_{0}$ and $\mathbb{E}_{1}$.

We insist that, for $x \in S^{3}$, both equations 4.12 and (4.13) involve the same relation of the parameter $\tau_{2}$ and the appropriate energy $\mathcal{E}_{2}$. Then we have

$$
\begin{aligned}
\left(u_{\mathrm{int}, i}-u_{\mathrm{ext}, i}\right)\left(\widetilde{x}^{2}+r_{\varepsilon} x\right)= & -4 \ln \tau_{2}+8 \lambda_{2} \ln r_{\varepsilon}|x|+H^{\mathrm{int}}\left(\varphi_{i}^{2}, \psi_{i}^{2}, x\right)-H^{\mathrm{ext}}\left(\widetilde{\varphi}_{i}^{2}, \widetilde{\psi}_{i}^{2}, x\right) \\
& -\left[(2-\gamma-\xi) H\left(\widetilde{x}^{2}, \widetilde{x}^{2}\right)+\frac{1-\xi}{\gamma} G\left(\widetilde{x}^{2}, \widetilde{x}^{1}\right)+\frac{1-\gamma}{\xi} G\left(\widetilde{x}^{2}, \widetilde{x}^{3}\right)\right] \\
& +\mathcal{O}\left(r_{\varepsilon}^{2}\right) .
\end{aligned}
$$

Projecting the set of equations (4.14) over $\mathbb{E}_{0}$, we get

$$
\begin{aligned}
-4 \ln \tau_{2}+8 \lambda_{2} \ln r_{\varepsilon}+\varphi_{i, 0}^{2}-\widetilde{\varphi}_{i, 0}^{2}-\mathcal{E}_{2}\left(\widetilde{x}^{2}, \widetilde{x}\right)+\mathcal{O}\left(r_{\varepsilon}^{2}\right) & =0, \\
8 \lambda_{2}+2 \varphi_{i, 0}^{2}+2 \widetilde{\varphi}_{i, 0}^{2}+\mathcal{O}\left(r_{\varepsilon}^{2}\right) & =0, \\
16 \lambda_{2}+8 \varphi_{i, 0}^{2}+\mathcal{O}\left(r_{\varepsilon}^{2}\right) & =0, \\
-32 \lambda_{2}+\mathcal{O}\left(r_{\varepsilon}^{2}\right) & =0,
\end{aligned}
$$

where

$$
\mathcal{E}_{2}(\cdot, \widetilde{\boldsymbol{x}}):=(2-\gamma-\xi) H\left(\cdot, \widetilde{x}^{2}\right)+\frac{1-\xi}{\gamma} G\left(\cdot, \widetilde{x}^{1}\right)+\frac{1-\gamma}{\xi} G\left(\cdot, \widetilde{x}^{3}\right) .
$$

The system 4.15 can be simply written as

$$
\lambda_{2}=\mathcal{O}\left(r_{\varepsilon}^{2}\right), \quad \varphi_{i, 0}^{2}=\mathcal{O}\left(r_{\varepsilon}^{2}\right), \quad \widetilde{\varphi}_{i, 0}^{2}=\mathcal{O}\left(r_{\varepsilon}^{2}\right) \quad \text { and } \quad \frac{1}{\ln r_{\varepsilon}}\left[4 \ln \tau_{2}+\mathcal{E}_{2}\left(\widetilde{x}^{2}, \widetilde{\boldsymbol{x}}\right)\right]=\mathcal{O}\left(r_{\varepsilon}^{2}\right)
$$

We are now in a position to define $\tau_{2}^{-}$and $\tau_{2}^{+}$. In fact, according to the above analysis, as $\varepsilon$ tends to 0 , we expect that $\widetilde{x}^{i}$ will converge to $x^{i}$ for $i \in\{1,2,3\}$ and $\tau_{2}$ will converge to $\tau_{2}^{*}$ satisfying

$$
4 \ln \tau_{2}^{*}=-\mathcal{E}_{2}\left(x^{2}, \boldsymbol{x}\right) .
$$

Hence it is enough to choose $\tau_{2}^{-}$and $\tau_{2}^{+}$in such a way that

$$
4 \ln \left(\tau_{2}^{-}\right)<-\mathcal{E}_{2}\left(x^{2}, \boldsymbol{x}\right)<4 \ln \left(\tau_{2}^{+}\right) .
$$

Consider now the projection of 4.14 over $\mathbb{E}_{1}$. Given a smooth function $f$ defined in $\Omega$, we identify its gradient $\nabla f=\left(\partial_{x_{1}} f, \ldots, \partial_{x_{4}} f\right)$ with the element of $\mathbb{E}_{1}$,

$$
\bar{\nabla} f=\sum_{i=1}^{4} \partial_{x_{i}} f e_{i} .
$$

Keeping these notations in mind, we obtain the system of equations

$$
\varphi_{i, 1}^{2}-\widetilde{\varphi}_{i, 1}^{2}-\bar{\nabla} \mathcal{E}_{2}\left(\widetilde{x}^{2}, \widetilde{\boldsymbol{x}}\right)+\mathcal{O}\left(r_{\varepsilon}^{2}\right)=0
$$




$$
\begin{aligned}
3 \varphi_{i, 1}^{2}+3 \widetilde{\varphi}_{i, 1}^{2}+\frac{1}{2} \widetilde{\psi}_{i, 1}^{2}-\bar{\nabla} \mathcal{E}_{2}\left(\widetilde{x}^{2}, \widetilde{\boldsymbol{x}}\right)+\mathcal{O}\left(r_{\varepsilon}^{2}\right)=0 \\
15 \varphi_{i, 1}^{2}-3 \widetilde{\varphi}_{i, 1}^{2}-\widetilde{\psi}_{i, 1}^{2}+\mathcal{O}\left(r_{\varepsilon}^{2}\right)=0 \\
15 \varphi_{i, 1}^{2}+15 \widetilde{\varphi}_{i, 1}^{2}+\frac{18}{4} \widetilde{\psi}_{i, 1}^{2}+\mathcal{O}\left(r_{\varepsilon}^{2}\right)=0
\end{aligned}
$$

which can be simplified as follows

$$
\varphi_{i, 1}^{2}=\mathcal{O}\left(r_{\varepsilon}^{2}\right), \quad \widetilde{\varphi}_{i, 1}^{2}=\mathcal{O}\left(r_{\varepsilon}^{2}\right), \quad \widetilde{\psi}_{i, 1}^{2}=\mathcal{O}\left(r_{\varepsilon}^{2}\right) \quad \text { and } \quad \bar{\nabla} \mathcal{E}_{2}\left(\widetilde{x}^{2}, \widetilde{\boldsymbol{x}}\right)=\mathcal{O}\left(r_{\varepsilon}^{2}\right)
$$

Finally, we consider the projection onto $L^{2}\left(S^{3}\right)^{\perp}$. This yields the system

$$
\begin{aligned}
& \varphi_{i}^{2, \perp}-\widetilde{\varphi}_{i}^{2, \perp}+\mathcal{O}\left(r_{\varepsilon}^{2}\right)=0, \partial_{r}\left(H_{\varphi_{i}^{2, \perp}, \psi_{i}^{2, \perp}}^{\mathrm{int}}-H_{\widetilde{\varphi}_{i}^{2, \perp}, \widetilde{\psi}_{i}^{2, \perp}}^{\mathrm{ext}}\right)+\mathcal{O}\left(r_{\varepsilon}^{2}\right)=0, \\
& \psi_{i}^{2, \perp}-\widetilde{\psi}_{i}^{2, \perp}+\mathcal{O}\left(r_{\varepsilon}^{2}\right)=0, \quad \partial_{r} \Delta\left(H_{\varphi_{i}^{2, \perp}, \psi_{i}^{2, \perp}}^{\mathrm{int}}-H_{\widetilde{\varphi}_{i}^{2, \perp}, \widetilde{\psi}_{i}^{2, \perp}}^{\mathrm{ext}}\right)+\mathcal{O}\left(r_{\varepsilon}^{2}\right)=0 .
\end{aligned}
$$

Thanks to the result of Lemma 3.8, this last system can be rewritten as

$$
\varphi_{i}^{2, \perp}=\mathcal{O}\left(r_{\varepsilon}^{2}\right), \quad \widetilde{\varphi}_{i}^{2, \perp}=\mathcal{O}\left(r_{\varepsilon}^{2}\right), \quad \psi_{i}^{2, \perp}=\mathcal{O}\left(r_{\varepsilon}^{2}\right) \quad \text { and } \quad \widetilde{\psi}_{i}^{2, \perp}=\mathcal{O}\left(r_{\varepsilon}^{2}\right) .
$$

If we define the parameter $t_{2} \in \mathbb{R}$ by

$$
t_{2}=\frac{1}{\ln r_{\varepsilon}}\left[4 \ln \tau_{2}+\mathcal{E}_{2}\left(\widetilde{x}^{2}, \widetilde{\boldsymbol{x}}\right)\right],
$$

then the systems found by projecting 4.14 gather in this equality

$$
T_{c, \varepsilon}^{2}=\left(t_{2}, \lambda_{2}, \varphi_{i, 0}^{2}, \widetilde{\varphi}_{i, 0}^{2}, \varphi_{i, 1}^{2}, \widetilde{\varphi}_{i, 1}^{2}, \widetilde{\psi}_{i, 1}^{2}, \bar{\nabla} \mathcal{E}_{2}\left(\widetilde{x}^{2}, \widetilde{\boldsymbol{x}}\right), \varphi_{i}^{2, \perp}, \widetilde{\varphi}_{i}^{2, \perp}, \psi_{i}^{2, \perp}, \widetilde{\psi}_{i}^{2, \perp}\right)=\mathcal{O}\left(r_{\varepsilon}^{2}\right)
$$

for $i=1,2$. As usual, the terms $\mathcal{O}\left(r_{\varepsilon}^{2}\right)$ depend nonlinearly on all the variables on the left side, but is bounded (in the appropriate norm) by a constant (independent of $\varepsilon$ and $\kappa$ ) times $r_{\varepsilon}^{2}$, provided $\varepsilon \in\left(0, \varepsilon_{\kappa}\right)$.

- On $\partial B_{r_{\varepsilon}}\left(\widetilde{x}^{3}\right)$, according to the proof of Theorem 1.5, using the fact that

$$
\begin{array}{ll}
\varphi_{1}^{3}=\varphi_{1,0}^{3}+\varphi_{1,1}^{3}+\varphi_{1}^{3, \perp}, & \psi_{1}^{3}=8 \varphi_{1,0}^{3}+12 \varphi_{1,1}^{3}+\psi_{1}^{3, \perp}, \\
\widetilde{\varphi}_{1}^{3}=\widetilde{\varphi}_{1,0}^{3}+\widetilde{\varphi}_{1,1}^{3}+\widetilde{\varphi}_{1}^{3, \perp}, & \widetilde{\psi}_{1}^{3}=\widetilde{\psi}_{1,1}^{3}+\widetilde{\psi}_{1}^{3, \perp}
\end{array}
$$

with $\varphi_{1,0}^{3}, \widetilde{\varphi}_{1,0}^{3} \in \mathbb{E}_{0}, \varphi_{1,1}^{3}, \widetilde{\varphi}_{1,1}^{3}, \widetilde{\psi}_{1,1}^{3} \in \mathbb{E}_{1}$ and $\varphi_{1}^{3, \perp}, \widetilde{\varphi}_{1}^{3, \perp}, \psi_{1}^{3, \perp}, \widetilde{\psi}_{1}^{3, \perp}$ belong to $\left(L^{2}\left(S^{3}\right)\right)^{\perp}$. We can prove that

$$
T_{1, \varepsilon}^{3}=\left(\varphi_{1,0}^{3}, \widetilde{\varphi}_{1,0}^{3}, \varphi_{1,1}^{3}, \widetilde{\varphi}_{1,1}^{3}, \widetilde{\psi}_{1,1}^{3}, \varphi_{1}^{3, \perp}, \widetilde{\varphi}_{1}^{3, \perp}, \psi_{1}^{3, \perp}, \widetilde{\psi}_{1}^{3, \perp}\right)=\mathcal{O}\left(r_{\varepsilon}^{2}\right) .
$$

On other hand, according to the proof of Theorem 1.5 and since when $\varepsilon$ tend to 0 , its enough to choose $\tau_{1}^{-}$and $\tau_{1}^{+}$is such a way that

$$
4 \ln \left(\tau_{3}^{-}\right)<-\ln \xi-\mathcal{E}_{3}\left(x^{3}, \boldsymbol{x}\right)<4 \ln \left(\tau_{3}^{+}\right),
$$


where

$$
\mathcal{E}_{3}(\cdot, \widetilde{\boldsymbol{x}}):=H\left(\cdot, \widetilde{x}^{3}\right)+G\left(\cdot, \widetilde{x}^{2}\right)+\frac{1-\xi}{\gamma} G\left(\cdot, \widetilde{x}^{1}\right) .
$$

Also using the fact that

$$
\begin{array}{ll}
\varphi_{2}^{3}=\varphi_{2,0}^{3}+\varphi_{2,1}^{3}+\varphi_{2}^{3, \perp}, & \psi_{2}^{3}=8 \varphi_{2,0}^{3}+12 \varphi_{2,1}^{3}+\psi_{2}^{3, \perp}, \\
\widetilde{\varphi}_{2}^{3}=\widetilde{\varphi}_{2,0}^{3}+\widetilde{\varphi}_{2,1}^{3}+\widetilde{\varphi}_{2}^{3, \perp}, & \widetilde{\psi}_{2}^{3}=\widetilde{\psi}_{2,1}^{3}+\widetilde{\psi}_{2}^{3, \perp},
\end{array}
$$

where $\varphi_{2,0}^{3}, \widetilde{\varphi}_{2,0}^{3} \in \mathbb{E}_{0}=\mathbb{R}$ are constant on $S^{3}, \varphi_{2,1}^{3}, \widetilde{\varphi}_{2,1}^{3}, \widetilde{\psi}_{2,1}^{3}$ belong to $\mathbb{E}_{1}=\operatorname{Span}\left\{e_{1}, e_{2}\right.$, $\left.e_{3}, e_{4}\right\}$ and $\varphi_{2}^{3, \perp}, \widetilde{\varphi}_{2}^{3, \perp}, \psi_{2}^{3, \perp}, \widetilde{\psi}_{2}^{3, \perp}$ are $L^{2}\left(S^{3}\right)$ orthogonal to $\mathbb{E}_{0}$ and $\mathbb{E}_{1}$. We can prove that

$$
\begin{aligned}
\left(u_{\mathrm{int}, i}-u_{\mathrm{ext}, i}\right)\left(\widetilde{x}^{3}+r_{\varepsilon} \cdot\right) & =0, \quad \partial_{r}\left(u_{\mathrm{int}, i}-u_{\mathrm{ext}, i}\right)\left(\widetilde{x}^{3}+r_{\varepsilon} \cdot\right)=0, \\
\Delta\left(u_{\mathrm{int}, i}-u_{\mathrm{ext}, i}\right)\left(\widetilde{x}^{3}+r_{\varepsilon} \cdot\right) & =0, \quad \partial_{r} \Delta\left(u_{\mathrm{int}, i}-u_{\mathrm{ext}, i}\right)\left(\widetilde{x}^{3}+r_{\varepsilon} \cdot\right)=0
\end{aligned}
$$

on $S^{3}$ yield to

$$
T_{2, \varepsilon}^{3}=\left(t_{3}, \lambda_{3}, \varphi_{2,0}^{3}, \widetilde{\varphi}_{2,0}^{3}, \varphi_{2,1}^{3}, \widetilde{\varphi}_{2,1}^{3}, \widetilde{\psi}_{2,1}^{3} \bar{\nabla} \mathcal{E}_{3}\left(\widetilde{x}^{3}, \widetilde{\boldsymbol{x}}\right), \varphi_{2}^{3, \perp}, \widetilde{\varphi}_{2}^{3, \perp}, \psi_{2}^{3, \perp}, \widetilde{\psi}_{2}^{3, \perp}\right)=\mathcal{O}\left(r_{\varepsilon}^{2}\right)
$$

where

$$
t_{3}=\frac{1}{\ln r_{\varepsilon}}\left[4 \ln \tau_{3}+\ln \xi+\mathcal{E}_{3}\left(\widetilde{x}^{3}, \widetilde{\boldsymbol{x}}\right)\right] .
$$

As usual, the terms $\mathcal{O}\left(r_{\varepsilon}^{2}\right)$ depend nonlinearly on all the variables on the left side, but is bounded (in the appropriate norm) by a constant (independent of $\varepsilon$ and $\kappa$ ) times $r_{\varepsilon}^{2}$, provided $\varepsilon \in\left(0, \varepsilon_{\kappa}\right)$.

We recall that $\boldsymbol{d}=r_{\varepsilon}(\widetilde{\boldsymbol{x}}-\boldsymbol{x})$, in addition the previous systems can be written as for $i=1,2,3$ :

$$
\left(\boldsymbol{d}, t_{i}, \lambda_{i}, \varphi^{i}, \widetilde{\varphi}^{i}, \psi^{i}, \widetilde{\psi}^{i}, \bar{\nabla} \mathcal{E}_{i}\right)=\mathcal{O}\left(r_{\varepsilon}^{2}\right) .
$$

Combining 4.10), 4.11), 4.16), 4.17) and (4.18), we have

$$
T_{i, \varepsilon}=\left(T_{i, \varepsilon}^{1}, T_{i, \varepsilon}^{2}, T_{i, \varepsilon}^{3}\right)=\left(\mathcal{O}\left(r_{\varepsilon}^{2}\right), \mathcal{O}\left(r_{\varepsilon}^{2}\right), \mathcal{O}\left(r_{\varepsilon}^{2}\right)\right) \quad \text { for } i=1,2 .
$$

Then the nonlinear mapping which appears on the right-hand side of 4.19 is continuous, compact. In addition, reducing $\varepsilon_{\kappa}$ if necessary, this nonlinear mapping sends the ball of radius $\kappa r_{\varepsilon}^{2}$ (for the natural product norm) into itself, provided $\kappa$ is fixed large enough. Applying Schauder's fixed point theorem in the ball of radius $\kappa r_{\varepsilon}^{2}$ in the product space where the entries live, we obtain the existence of a solution of equation (4.19). This completes the proof of Theorem 1.6 .

\section{References}

[1] G. Arioli, F. Gazzola, H.-C. Grunau and E. Mitidieri, A semilinear fourth order elliptic problem with exponential nonlinearity, SIAM J. Math. Anal. 36 (2005), no. 4, 1226-1258. 
[2] S. Baraket, I. Bazarbacha and N. Trabelsi, Construction of singular limits for fourdimensional elliptic problems with exponentially dominated nonlinearity, Bull. Sci. Math. 131 (2007), no. 7, 670-685.

[3] S. Baraket, I. Ben Omrane, T. Ouni and N. Trabelsi, Singular limits for 2-dimensional elliptic problem with exponentially dominated nonlinearity and singular data, Commun. Contemp. Math. 13 (2011), no. 4, 697-725.

[4] S. Baraket, M. Dammak, T. Ouni and F. Pacard, Singular limits for a 4-dimensional semilinear elliptic problem with exponential nonlinearity, Ann. Inst. H. Poincaré Anal. Non Linéaire 24 (2007), no. 6, 875-895.

[5] S. Baraket, A. Messaoudi, S. Saanouni and N. Trabelsi, Blow-up in coupled solutions for a 4-dimensional semilinear elliptic system of Liouville type, to appear in Adv. Pure Appl. Math.

[6] S. Baraket and F. Pacard, Construction of singular limits for a semilinear elliptic equation in dimension 2, Calc. Var. Partial Differential Equations 6 (1998), no. 1, $1-38$.

[7] S. Baraket, S. Sâanouni and N. Trabelsi, Singular limit solutions for a 2-dimensional semilinear elliptic system of Liouville type in some general case, Discrete Contin. Dyn. Syst. 40 (2020), no. 2, 1013-1063.

[8] S. Baraket and D. Ye, Singular limit solutions for two-dimentional elliptic problems with exponentially dominated nonlinearity, Chin. Ann. Math. Ser. B 22 (2001), no. 3, $287-296$.

[9] S.-Y. A. Chang, On a fourth-order partial differential equation in conformal geometry, in: Harmonic Analysis and Partial Differential Equations (Chicago, IL, 1996), 127150, Chicago Lectures in Mathematics, University of Chicago Press, Chicago, IL, 1999.

[10] S. Chanillo and M. K.-H. Kiessling, Conformally invariant systems of nonlinear PDE of Liouville type, Geom. Funct. Anal. 5 (1995), no. 6, 924-947.

[11] M. Clapp, C. Muñoz and M. Musso, Singular limits for the bi-Laplacian operator with exponential nonlinearity in $\mathbb{R}^{4}$, Ann. Inst. H. Poincaré Anal. Non Linéaire 25 (2008), no. 5, 1015-1041.

[12] M. Dammak and T. Ouni, Singular limits for 4-dimensional semilinear elliptic problems with exponential nonlinearity adding a singular source term given by Dirac masses, Differential Integral Equations 21 (2008), no. 11-12, 1019-1036. 
[13] Z. Djadli and A. Malchiodi, Existence of conformal metrics with constant Q-curvature, Ann. of Math. (2) 168 (2008), no. 3, 813-858.

[14] P. Esposito, Blow up solutions for a Liouville equation with singular data, in: Recent Advances in Elliptic and Parabolic Problems, 61-79, World Sci. Publ., Hackensack, NJ, 2005.

[15] C.-S. Lin, A classification of solutions of a conformally invariant fourth order equation in $\mathbb{R}^{n}$, Comment. Math. Helv. 73 (1998), no. 2, 206-231.

[16] C.-S. Lin, J. Wei and D. Ye, Classification and nondegeneracy of $S U(n+1)$ Toda system with singular sources, Invent. Math. 190 (2012), no. 1, 169-207.

[17] C.-S. Lin, J.-c. Wei and L. Zhang, Classification of blowup limits for SU(3) singular Toda systems, Anal. PDE 8 (2015), no. 4, 807-837.

[18] J. Liouville, Sur l'équation aux différences partielles $\partial^{2} \log \frac{\lambda}{\partial u \partial v} \pm \frac{\lambda}{2 a^{2}}=0$, J. Math. 18 (1853), 17-72.

[19] M. Musso, A. Pistoia and J. Wei, New blow-up phenomena for $S U(n+1)$ Toda system, J. Differential Equations 260 (2016), no. 7, 6232-6266.

[20] J. Wei, Asymptotic behavior of a nonlinear fourth order eigenvalue problem, Comm. Partial Differential Equations 21 (1996), no. 9-10, 1451-1467.

[21] J. Wei and D. Ye, Nonradial solutions for a conformally invariant fourth order equation in $\mathbb{R}^{4}$, Calc. Var. Partial Differential Equations 32 (2008), no. 3, 373-386.

Sami Baraket, Imen Bazarbacha and Rima Chetouane

University of Tunis El Manar, Department of Mathematics, Faculty of Sciences of Tunis, Campus of University 2092 Tunis El Manar, Tunisia

E-mail address: smbaraket@yahoo.fr, imen.bazarbacha@gmail.com,

rima.chetouane@fst.utm.tn

Abdellatif Messaoudi

University of Tunis El Manar Preparatory Institute for Engineering Studies - El Manar BP 244 Tunis El Manar 2092 Tunisia

E-mail address: messaoudi.abdellatif@gmail.com 